\title{
1 ARID1A maintains transcriptionally repressive H3.3 associated with CHD4-ZMYND8
}

\section{2 chromatin interactions.}

3

4 Jake J. Reske ${ }^{1}$, Mike R. Wilson ${ }^{1}$, Brooke Armistead ${ }^{1}$, Cristina Perez ${ }^{1}$, Joel Hrit ${ }^{2}$, Marie Adams ${ }^{3}$,

5 Scott B. Rothbart ${ }^{2}$, Stacey A. Missmer ${ }^{1,4}$, Asgerally T. Fazleabas ${ }^{1,4}$, and Ronald L. Chandler ${ }^{1,2,4^{*}}$

6

$7 \quad{ }^{1}$ Department of Obstetrics, Gynecology and Reproductive Biology, College of Human Medicine,

8 Michigan State University, Grand Rapids, MI 49503, USA.

$9 \quad{ }^{2}$ Department of Epigenetics, Van Andel Research Institute, Grand Rapids, MI 49503, USA.

$10{ }^{3}$ Genomics Core Facility, Van Andel Research Institute, Grand Rapids, MI 49503, USA.

11 4Department of Women's Health, Spectrum Health System, Grand Rapids, MI 49341, USA.

12

$13 *$ Corresponding author, rlc@msu.edu 
Reske et al.

\section{Abstract}

ARID1A is a signature subunit of the mammalian SWI/SNF (BAF) chromatin remodeling

17 complex and is mutated at a high rate in malignancies and benign diseases originating from the

18 uterine endometrium. Through genome-wide analysis of human endometriotic epithelial cells, we

19 show that more than half of ARID1A binding sites are marked by the variant histone H3.3,

20 including active regulatory elements. ARID1A loss leads to H3.3 depletion at ARID1A bound

21 active regulatory elements and a concomitant redistribution of H3.3 towards genic elements.

22 ARID1A interactions with the repressive chromatin remodeler CHD4 (NuRD) are associated with

23 H3.3-containing chromatin regulation. ZMYND8, the CHD4-interacting acetyl-histone H4 reader,

24 specifies ARID1A-CHD4-H3.3 target regulatory activity towards histone H4 lysine 16 acetylation

25 (H4K16ac) to repress super-enhancers. ARID1A, H3.3, CHD4, and ZMYND8 co-repress the

26 expression of genes governing extracellular matrix, motility, adhesion, and epithelial-to-

27 mesenchymal transition. Moreover, these gene expression alterations are observed in human

28 endometriomas. Altogether, these studies demonstrate that cooperation among a histone reader

29 and different types of chromatin remodelers safeguards the endometrium through transcriptionally

30 repressive $\mathrm{H} 3.3$.

31 
Reske et al.

32

33

34

35

36

37

\section{Introduction}

The SWI/SNF complex remodels chromatin through ATP-dependent DNA sliding, H2A/H2B dimer eviction, and nucleosome ejection functions (Dechassa et al., 2010; Kassabov, Zhang, Persinger, \& Bartholomew, 2003; Whitehouse et al., 1999). SWI/SNF remodeling activities open chromatin and promote accessibility for other DNA-binding factors and chromatin regulators (Clapier, 2021; Clapier \& Cairns, 2009). SWI/SNF complex composition is heterogeneous and cell type dependent (Wang et al., 1996). SWI/SNF regulates lineage-specific enhancer activity through multiple mechanisms (Alver et al., 2017). Protein subunit architecture contributes to SWI/SNF complex specificity through specialized cofactor interactions. The activities of chromatin remodelers and associated machinery are known to modulate the epigenome by regulating histone post-translational modifications and nucleosome composition (Clapier, 2021). Multiple chromatin remodeler complexes are often observed at the same genomic loci and can perform redundant, cooperative, or antagonistic transcriptional regulatory roles (Morris et al., 2014).

Subunits within the mammalian SWI/SNF (BAF) chromatin remodeler complex are mutated across an estimated 20\% of all human cancers (Kadoch et al., 2013). Tissue-specific propensities for mutations in certain SWI/SNF subunits are also evident (Kadoch \& Crabtree, 2015). ARID1A (BAF250A) is the most frequently mutated SWI/SNF subunit (Mittal \& Roberts, 2020). ARID1A is the largest SWI/SNF subunit and acts as a structural scaffold for other subunits in certain SWI/SNF complexes (He et al., 2020; Mashtalir et al., 2018). ARID1A also exhibits essential DNA-binding activity albeit in a non-sequence-specific manner (Chandler et al., 2013; Dallas et al., 2000). Defects in chromatin accessibility and higher-order chromatin structure are

4 thought to underlie ARID1A and SWI/SNF mutant pathogenesis at least partially (Barutcu et al., 
Reske et al.

2016; Kelso et al., 2017). Uterine endometrial cancer displays high rates of ARID1A mutation, with roughly $40 \%$ of cases showing loss of ARID1A expression (Cancer Genome Atlas Research

57 et al., 2013; Wu, Wang, \& Shih Ie, 2014). ARID1A mutations and loss of expression are also

58 observed in deeply invasive forms of endometriosis, which is characterized by ectopic spread of

59 the endometrium (Anglesio et al., 2017; Samartzis et al., 2012; Zondervan, Becker, \& Missmer,

60 2020). ARID1A mutations are also common in endometriosis-associated ovarian cancers (Jones

61 et al., 2010; Wiegand et al., 2010).

In the endometrial epithelium, we have previously shown that ARID1A normally promotes epithelial identity by repressing the expression of mesenchymal and invasion genes, at least in part

64 through promoter-proximal and distal chromatin interactions that affect transcriptional activity

65 (Reske et al., 2020; Wilson et al., 2020; Wilson et al., 2019). Other reports have demonstrated that ARID1A and SWI/SNF can function as a repressor, often through interactions with repressive

67 machinery (Bui et al., 2019; Chandler et al., 2013; Rafati et al., 2011; Van Rechem, Boulay, \& Leprince, 2009). Although nucleosome structure and histone post-translational modifications are suspected mechanisms, it remains poorly understood how SWI/SNF governs the epigenome.

71 chromatin that is associated with biochemical and genomic interactions with the SWI/SNF-like

72 CHD4 (NuRD) remodeler complex. This regulation is further specified toward repression of

73 transcriptional hyperactivation through CHD4 interactions with the multivalent histone reader

74 ZMYND8 to target H4K16 acetylated regions of the genome, including a subset of super-

75 enhancers. We finally reveal that this mechanism of ARID1A, H3.3, CHD4, and ZMYND8 co-

76 repression targets pathophysiological genes involved in epithelial-to-mesenchymal transition

77 (EMT) and cellular invasion, and these genes are aberrantly upregulated in human endometriomas. 
Reske et al.

78 Altogether, our studies reveal a role for ARID1A-containing SWI/SNF complexes in the

79 maintenance of H3.3, and, at a subset of physiologically relevant target genes, ARID1A-CHD4ZMYND8 interactions govern transcriptionally repressive H3.3.

\section{Results}

\section{ARID1A mutations in cancer are associated with reduced histone H3.3.}

85 the endometrium, we leveraged the Cancer Cell Line Encyclopedia (CCLE) global chromatin

86 profiling data set of bulk histone H3 peptide measurements by mass spectrometry (Ghandi et al.,

87 2019). Among the 896 total cancer cell lines that were assayed along with genetic mutation

88 profiling, 27 are specifically endometrial cancer. Cancer cell lines were segregated by ARID1A

89 mutation status, and relative bulk H3 peptide abundances were compared between ARID1A

90 mutant and wild-type cancer cell lines (Figure 1A). This resource and analysis allowed us to

91 identify histone peptide abundances associated with ARID1A mutation. Across cancer and

92 specifically within endometrial cancer, we observed that ARID1A mutant lines showed overall

93 higher levels of H3K27ac1K36me0 and H3K79me2 and lower levels of H3K4me1, H3K4me2,

94 and H3.3 (H3.3K27me0K36me0) (Figure 1B). Consistent with prior literature, SWI/SNF (BAF)

95 was shown to biophysically and functionally regulate H3K4me1-marked chromatin (Local et al.,

96 2018). Histone $\mathrm{H} 3.3$ is a variant of canonical H3 with known ties to active chromatin and

97 transcriptional regulation (Ahmad \& Henikoff, 2002; Shi, Wen, \& Shi, 2017; Szenker, Ray-Gallet,

98 \& Almouzni, 2011). Like SWI/SNF, H3.3 has also been observed to mark and regulate active

99 enhancers (Chen et al., 2013; Deaton et al., 2016; Martire et al., 2019; H. Zhang et al., 2017). In

100 contrast with H3.3, abundance of the respective canonical H3 (H3.1) peptide was not different 
bioRxiv preprint doi: https://doi.org/10.1101/2022.02.27.482165; this version posted February 28, 2022. The copyright holder for this preprint (which was not certified by peer review) is the author/funder, who has granted bioRxiv a license to display the preprint in perpetuity. It is made available under aCC-BY 4.0 International license.

Reske et al.

between ARID1A mutant and wild-type lines (Figure 1C-D). To rule out the possibility that cancer-associated histone gene mutations could cause this phenomenon, we recapitulated these H3.3 associations in cell lines that are wild-type for all 74 human histone genes (Nacev et al., 2019) (Figure 1-figure supplement 1). While a minor effect size, the association between pathogenic chromatin alterations following ARID1A mutation.

Figure 1. Histone peptide abundance associated with ARID1A mutation in cancer cell lines.

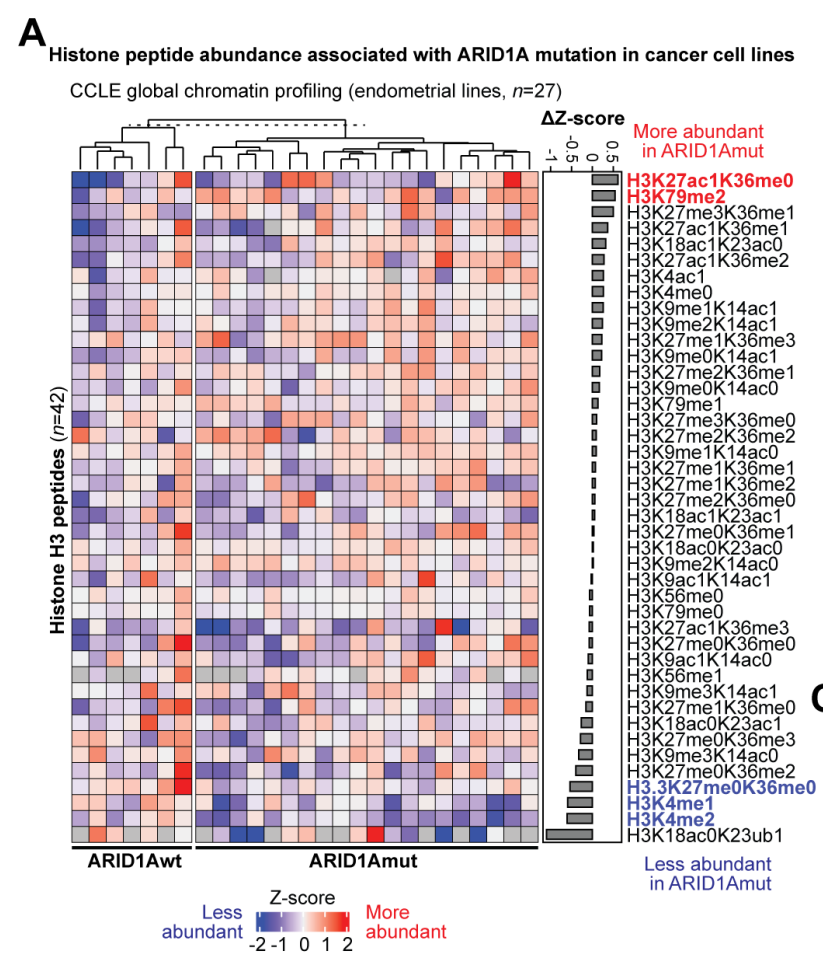

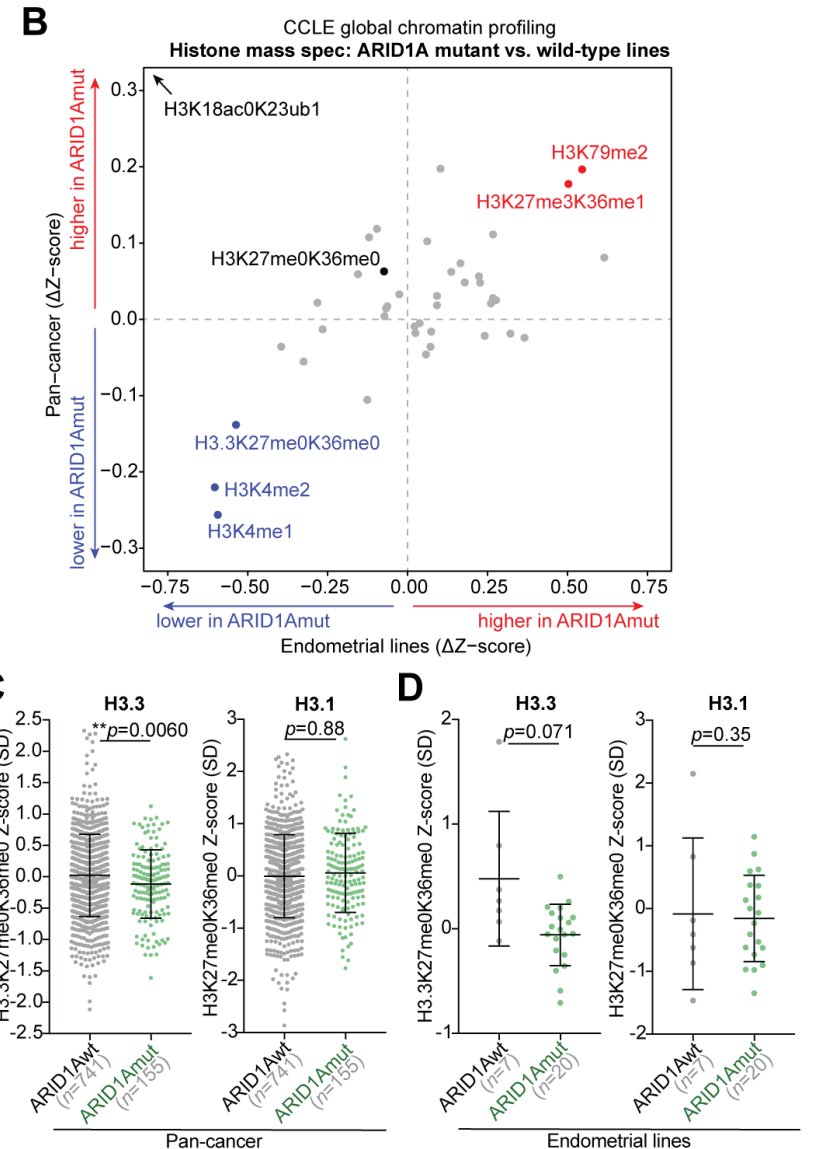

A, Representation of 42 distinct histone $\mathrm{H} 3$ peptide measurements by mass spectrometry in public CCLE global chromatin profiling data, specifically for endometrial cancer cell lines stratified by 
Reske et al.

113 ARID1A mutation status. Heatmap values are relative peptide abundance Z-scores, where 0

114 (white) is the mean across all pan-cancer cell lines. H3 peptides were then ranked (y-axis) by

115 differential abundance between ARID1A mutant vs. wild-type lines. B, Summary of ARID1A

116 mutant peptide associations across all cancer cell lines $(n=896, y$-axis $)$ or specifically endometrial

117 cancer lines $(n=27, x$-axis $)$. C-D, Box dot plots showing relative abundance of H3.3 vs. canonical

118 H3.1 peptides in ARID1A wild-type and mutant lines: C, pan-cancer lines; D, endometrial lines.

119 Statistic is two-tailed, unpaired Welch's $t$-test.

Figure 1-figure supplement 1. H3.3 analysis in histone wild-type lines.
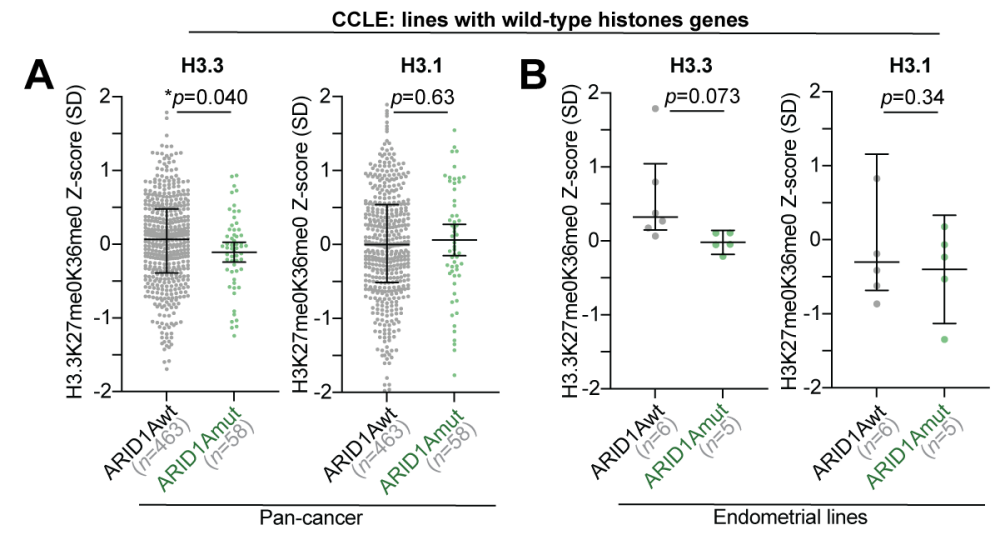

123 Repeated analysis of H3.3 and H3.1 abundance in CCLE lines that are wild-type for all 74 human

124 histone genes annotated by Nacev et al. Statistic is two-tailed, unpaired Welch's $t$-test. A, H3.3

125 (left) and H3.1 (right) peptide abundances in ARID1A mutant vs. wild-type pan-cancer lines, as

126 in Figure 1C. B, H3.3 (left) and H3.1 (right) peptide abundances in ARID1A mutant vs. wild-type

127 endometrial cancer lines, as in Figure 1D. 
Reske et al.

\section{ARID1A regulates $\mathrm{H3.3-associated} \mathrm{active} \mathrm{chromatin.}$}

We next interrogated the chromatin regulatory roles of $\mathrm{H} 3.3$ in immortalized $12 \mathrm{Z}$ human epithelial characteristics in $12 \mathrm{Z}$ endometriotic epithelial cells at both transcriptional and phenotypic levels, such that ARID1A loss leads to epithelial-to-mesenchymal transition (EMT) and enhanced migration and invasion (Wilson et al., 2020; Wilson et al., 2019). ARID1A loss in $12 \mathrm{Z}$ recapitulates many of the molecular and cellular features observed in ARID1A-deficient endometrial epithelia in vivo (Wilson et al., 2020; Wilson et al., 2019). Therefore, $12 \mathrm{Z}$ cells represent a model system to explore physiological roles for ARID1A in epigenomic regulation. immunoprecipitation followed by sequencing (ChIP-seq) in control $12 \mathrm{Z}$ cells ( $n=2$ IP replicates). Significant H3.3 enrichment was observed at 40,006 genomic regions (Figure 2A). Intronic,

141 intergenic, and promoter regions comprised the vast majority of H3.3 enrichment sites (Figure 142 2A), which may be expected as H3.3 is known to mark active regulatory elements such as 143 enhancers and gene promoters (Chen et al., 2013). H3.3 ChIP-seq peaks were 1830 bp in width on 144 average and ranged from $<500 \mathrm{bp}$ to $>10$ kilobases (Figure 2B). Intersecting H3.3 ChIP-seq peaks 145 with our previously published ARID1A ChIP-seq data from these cells (Wilson et al., 2019) 146 revealed that over half of each peak set overlapped, suggesting widespread co-regulation (Figure $1472 \mathrm{C})$. loss in $12 \mathrm{Z}$ cells via chromHMM (Ernst \& Kellis, 2012) by measuring seven chromatin features associated with transcriptional regulation: total RNA, ATAC (accessibility), H3K27ac, H3K18ac,

151 H3K4me1, H3K4me3, and H3K27me3 (Wilson et al., 2020). Similar to our previous reports of 
Reske et al.

ARID1A regulated chromatin states, genomic H3.3 enrichment was highly associated with all

153 active, euchromatic features, but not heterochromatic H3K27me3 (Figure 2D). Annotating H3.3

154 enrichment in each of our characterized chromatin states revealed that H3.3 is associated with

155 similar regulatory chromatin states as ARID1A binding, most notably super-enhancers and active

156 typical enhancers (Figure 2E, left). In agreement, co-regulation by H3.3 and ARID1A was most

157 prominently observed at these same chromatin states (Figure 2E, center). Next, we examined

ARID1A binding at H3.3-marked vs. H3.3-absent chromatin sub-states and found that ARID1A

binding was associated with H3.3 at promoter and genic super-enhancers and active transcription start sites (TSS) (Figure 2E, right).

Upon further investigation of ARID1A and H3.3 genome-wide binding patterns, we

162

163

164

165

166

167

168

169

170

171

172

173

174

observed that genome-wide ARID1A peaks showed overall stronger ARID1A binding when H3.3

was also localized, and H3.3 was overall more abundant at genome-wide H3.3 peaks also bound by ARID1A (Figure 2F). ARID1A and H3.3 ChIP-seq peaks were then broadly classified into promoter-proximal (located within $3 \mathrm{~kb}$ of a TSS) and distal (located further than $3 \mathrm{~kb}$ from a TSS). Interestingly, promoter-proximal ARID1A peaks were more likely to show H3.3 co-marking than distal ARID1A peaks, whereas distal H3.3 peaks were more likely to show ARID1A cobinding than promoter-proximal H3.3 peaks (Figure 2G). Further, ARID1A and H3.3 peaks were segregated into four classes based on binding status at promoter vs. distal sites. ARID1A binding was strongest at distal peaks marked by H3.3, while H3.3 was most abundant at promoter-proximal peaks bound by ARID1A (Figure 2H). These genome-wide binding patterns indicate that ARID1A and H3.3 may co-regulate active chromatin elements like enhancers and promoters.

We previously reported that ARID1A chromatin binding near gene promoters is associated with transcriptional regulation, such that ARID1A loss leads to aberrant gene expression (Wilson 
Reske et al.

175 et al., 2019). Our H3.3 data further revealed that ARID1A promoter binding is highly enriched 176 among genes marked by promoter H3.3 (Figure 2I, top), indicating that ARID1A transcriptional

177 regulation may be coupled with H3.3. Moreover, the 2037 genes co-marked by ARID1A and H3.3

178 in the promoter region were more likely to show differential expression (DE) following ARID1A

179 loss than genes without promoter H3.3 (Figure 2I, bottom). In addition, locus-scale investigation

180 clearly showed that ARID1A and H3.3 often co-mark active chromatin regulatory elements, which

181 infrequently also includes gene body coating by H3.3, such as at COL1A1, THBS1, and SERPINE1

182 (Figure 2J). These data collectively suggest H3.3 may be linked to transcriptional regulatory 183 activity by ARID1A at the level of chromatin. 
Reske et al.

Figure 2. Genome-wide analysis of H3.3-ARID1A chromatin co-regulation.
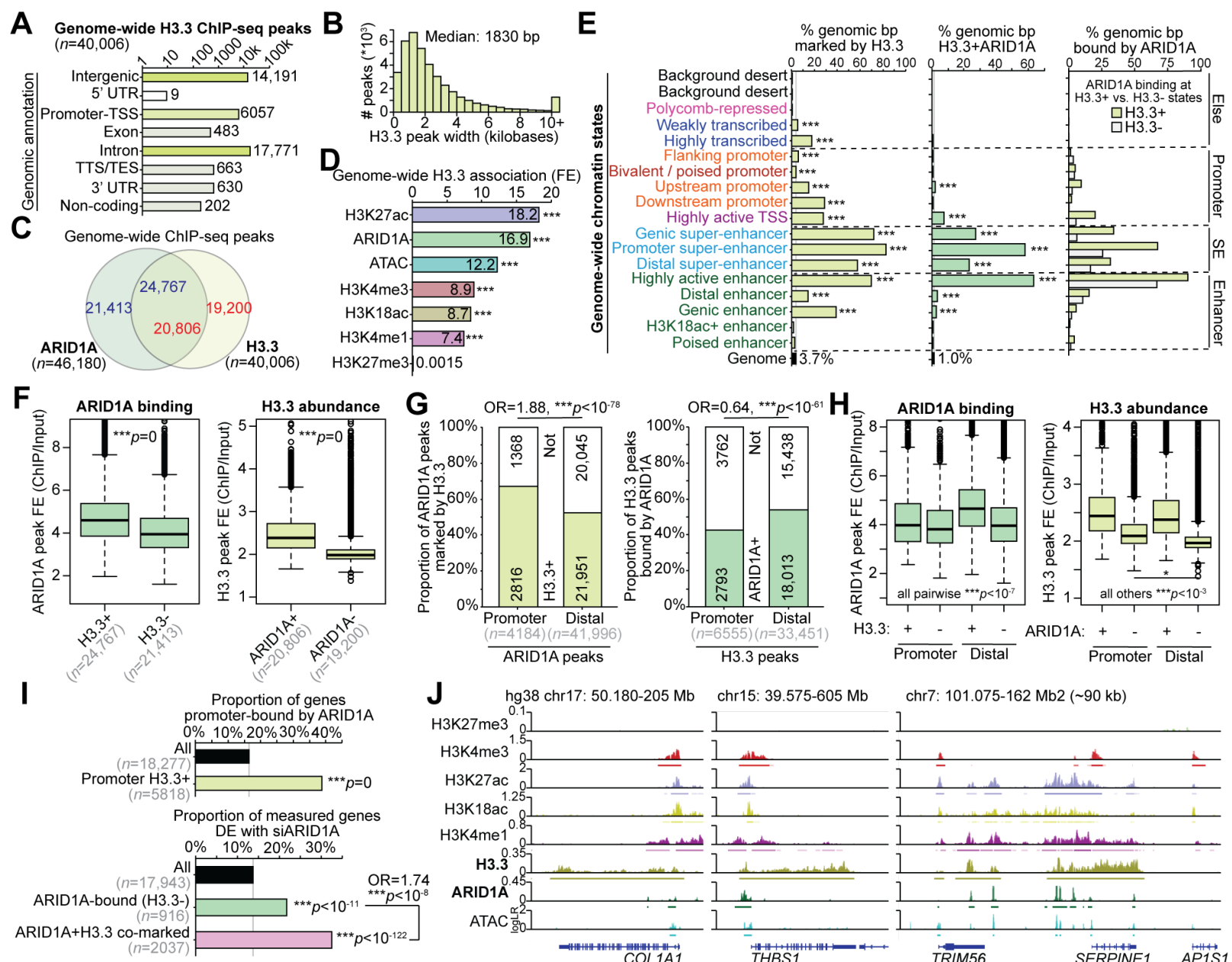

186
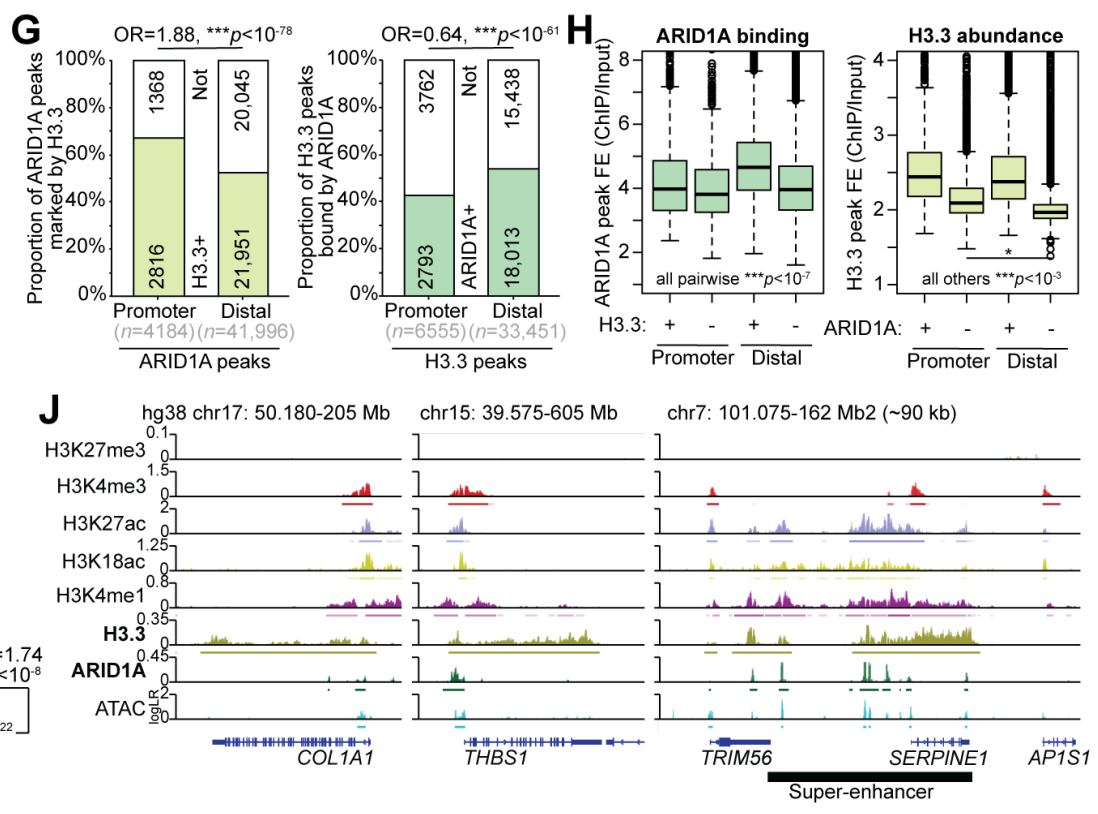

A, Genomic annotation of 40,006 genome-wide H3.3 ChIP-seq peaks in 12Z cells. B, Distribution of H3.3 peak widths. Median H3.3 peak width is 1830 bp. C, Genome-wide overlap of ARID1A and H3.3 ChIP-seq peaks. D, Genome-wide association between H3.3 and other previously measured chromatin features, per genomic bp. Statistic is hypergeometric enrichment. E,

191 Enrichment for H3.3 and ARID1A co-regulation across 18 chromatin states previously modeled via ChromHMM (Wilson et al., 2020). Left, enrichment of H3.3 peaks; center, enrichment of H3.3+ARID1A binding; right, enrichment of ARID1A binding at sites with vs. without H3.3. 
Reske et al.

enrichment, FE) at H3.3+ vs. H3.3- ARID1A peaks. Right, H3.3 abundance (ChIP/input foldenrichment) at ARID1A+ vs. ARID1A- H3.3 peaks. Statistic is two-tailed, unpaired Wilcoxon's test. G, Association between ARID1A and H3.3 co-binding at promoter proximal $(<3 \mathrm{~kb}$ from a TSS) vs. distal (>3 kb from a TSS) peaks. Statistic is two-tailed Fisher's exact test. H, Left, ARID1A binding levels at promoter vs. distal peaks with or without H3.3. Right, H3.3 abundance at promoter vs. distal peaks with or without ARID1A. Statistic is two-tailed, unpaired Wilcoxon's test. I, Top, enrichment of H3.3 at genes promoter-bound by ARID1A. Bottom, enrichment of ARID1A+H3.3 co-binding at genes DE following ARID1A loss (siARID1A treatment). Statistics are hypergeometric enrichment test and pairwise two-tailed Fisher's exact test. J, Example hg38

204 browser shots of genes and regulatory elements co-regulated by H3.3 and ARID1A. $y$-axis is log205 likelihood ratio (logLR) of assay signal (compared to input chromatin for ChIP-seq or background 206 genome for ATAC-seq). Small bars under tracks indicate significant peak detection by MACS2 207 (FDR < 0.05). Super-enhancers were detected by ROSE from H3K27ac ChIP-seq. * $p<0.05, * *$ $p<0.01, * * * p<0.001$. 
Reske et al.

\section{ARID1A chromatin interactions maintain H3.3.} by ChIP-seq. Our differential H3.3 ChIP-seq analysis (shARID1A vs. non-targeting shRNA control, $n=2$ ) indicated that nearly $1 / 3$ of tested H3.3 regions showed significant differences in H3.3 abundance $(c s a w / e d g e R, F D R<0.05)$ at 72 hours following ARID1A knockdown (Figure result in obvious changes in global H3.3 levels by immunoblotting (Figure 3-figure supplement indicating that the dominantly expressed $\mathrm{H} 3.3$-encoding gene isoform, $H 3 F 3 B$, does not change in expression (Figure 3-figure supplement 1C). Genomic regions that showed significantly affected

227 ARID1A binding, 81\% of the 8418 shARID1A decreasing H3.3 regions were normally bound by incorporation or maintain its stability.

We further characterized the changes in H3.3 occurring following ARID1A loss. Globally, 
Reske et al.

233 a TSS and excluding super-enhancers) were enriched for shARID1A-driven H3.3 alterations as

234 compared to gene promoters and super-enhancers (Figure 3-figure supplement 1D). Intriguingly,

235 gene promoters displayed both decreasing and increasing H3.3, whereas typical enhancers and

236 super-enhancers almost exclusively lost H3.3 if significantly affected (Figure 3-figure

237 supplement 1E). Among genomic H3.3 regions that were directly affected by ARID1A loss,

238 decreasing H3.3 regions tended to display greater differences in H3.3 abundance than increasing

239 H3.3 regions (Figure 3E). Regions that displayed decreasing H3.3 also tended to have overall

240 wider genomic footprints than increasing or stable H3.3 regions (Figure 3F). In agreement with

241 where ARID1A-H3.3 co-regulation is most frequently observed, chromatin state enrichment

242 analysis indicated that ARID1A loss led to depletion of H3.3 at promoter super-enhancers and

243 highly active enhancers, while increasing H3.3 was observed over actively transcribed gene bodies

244 (Figure 3G). From the 412 genes we identified with shARID1A direct decreasing promoter H3.3,

245 we found significant enrichment for inflammatory, hypoxia, apoptosis, locomotion, and EMT

246 pathways, such as CCL2 (Figure 3H-I). These data suggest that ARID1A maintains H3.3 at active

247 regulatory elements such as enhancers and super-enhancers, and, when ARID1A is lost,

248 redistribution of $\mathrm{H} 3.3$ occurs towards active genes already marked by H3.3. 
bioRxiv preprint doi: https://doi.org/10.1101/2022.02.27.482165; this version posted February 28, 2022. The copyright holder for this preprint (which was not certified by peer review) is the author/funder, who has granted bioRxiv a license to display the preprint in perpetuity. It is made available under aCC-BY 4.0 International license.

Reske et al.

Figure 3. Genome-wide analysis of ARID1A-dependent H3.3.

A

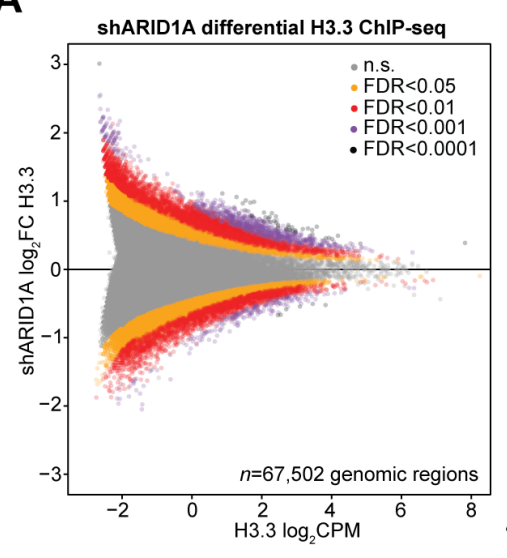

B

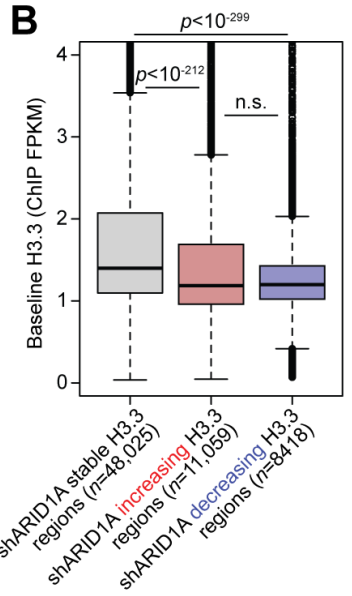

E
D

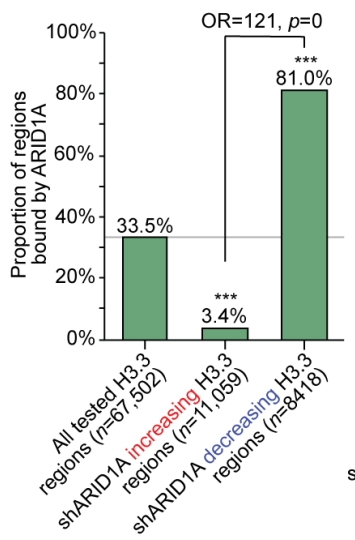

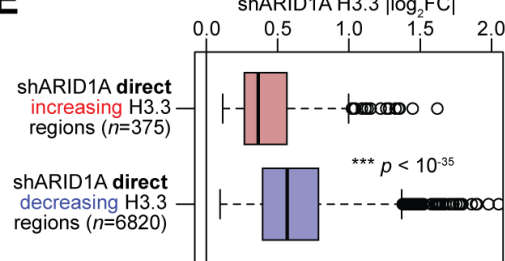

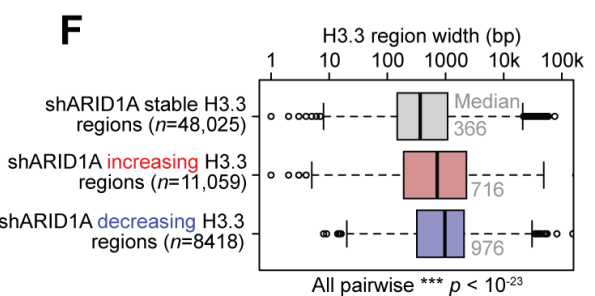

C

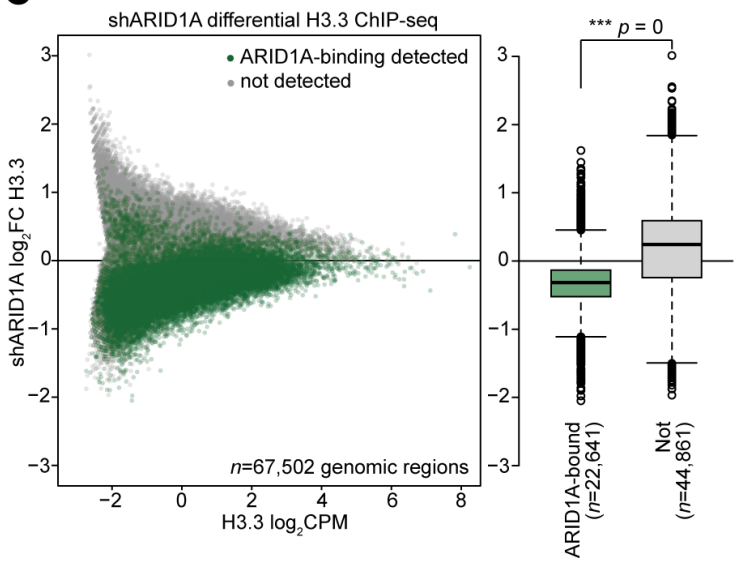

G
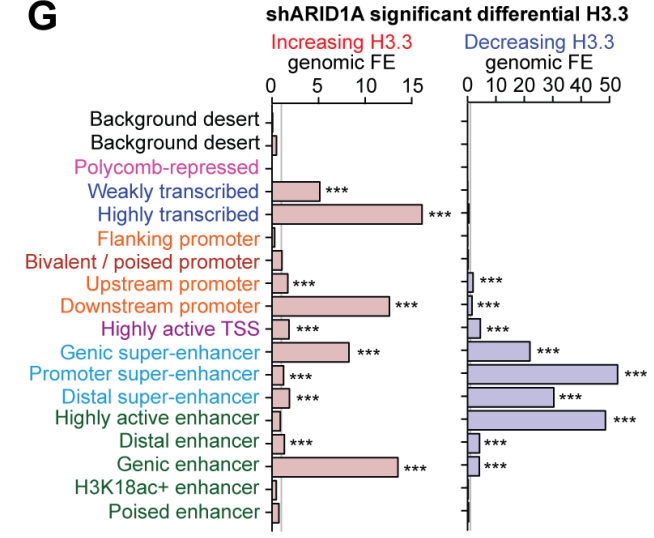

Poised enhancer -

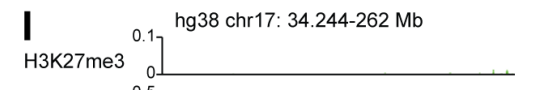

H Top enriched gene sets among shARID1A direct decreasing promoter H3.3 genes $(n=412)$
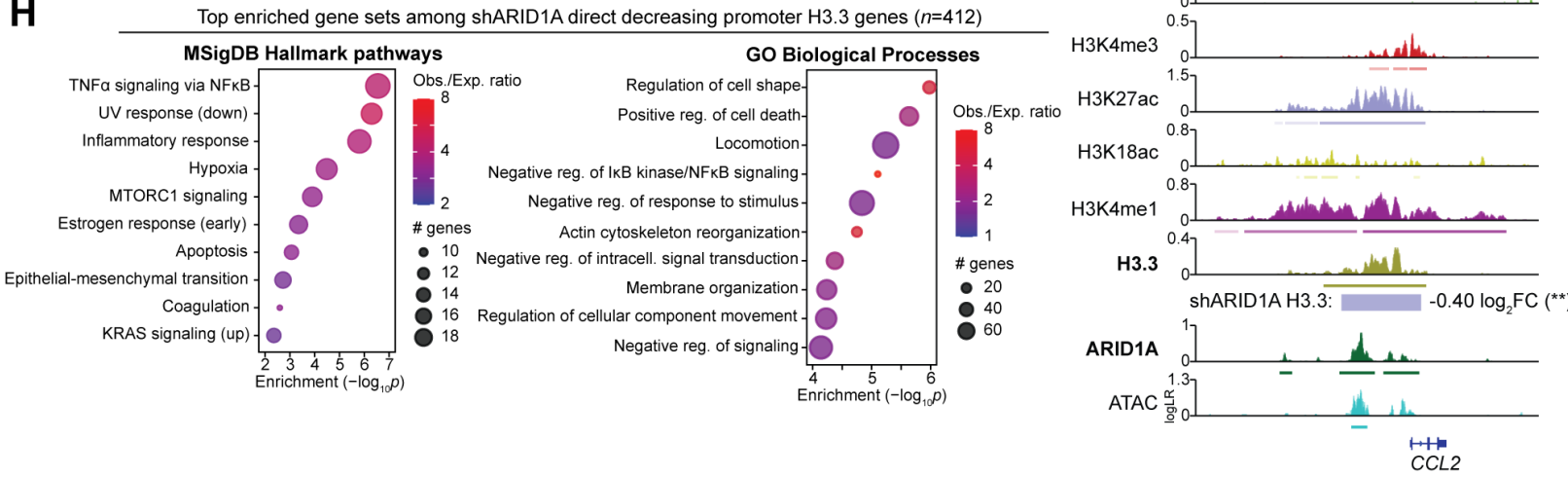

252 A, MA plot of shARID1A vs. control differential H3.3 ChIP-seq, $n=67,502$ tested genomic regions. Regions are colored based on shARID1A differential H3.3 significance. FDR $<0.05$ was

254 used as the significance threshold for all downstream analyses. B, Baseline H3.3 abundance (ChIP 
Reske et al.

256 H3.3, and regions that display decreasing H3.3. Statistic is two-tailed, unpaired Wilcoxon's test.

257 C, shARID1A differential H3.3 regions segregated by detection of ARID1A binding in wild-type

258 cells. Left, MA plot with all genome-wide H3.3 tested regions, colored by ARID1A binding status.

259 Right, box plot quantification of shARID1A $\log _{2} \mathrm{FC}$ H3.3 abundance, segregated by ARID1A

260 binding status. Statistic is two-tailed, unpaired Wilcoxon's test. D, Enrichment of ARID1A

261 binding detection at regions with decreasing H3.3 following ARID1A loss compared to all tested

262 H3.3 regions. Statistics are hypergeometric enrichment test and pairwise two-tailed Fisher's exact

263 test. E, Magnitude of $\mathrm{H} 3.3$ change $\left(\log _{2} \mathrm{FC}\right)$ among ARID1A-bound (direct) shARID1A

264 significantly decreasing vs. increasing H3.3 regions. Statistic is two-tailed, unpaired Wilcoxon's

265 test. F, Distribution of H3.3-enriched region widths among shARID1A stable vs. increasing vs.

266 decreasing H3.3 regions. Statistic is two-tailed, unpaired Wilcoxon's test. G, Chromatin state

267 enrichment among shARID1A increasing and decreasing H3.3 regions, calculated per 200 bp

268 genomic interval. Statistic is hypergeometric enrichment. H, Top 10 significant $($ FDR $<0.05)$

269 enriched Hallmark pathways (left) and GO Biological Process gene sets (right) among genes with

270 shARID1A direct (ARID1A-bound) decreasing promoter H3.3. I, Representative hg38 locus near

271 CCL2 displaying H3.3 maintained by ARID1A chromatin interactions. *** $p<0.001$.

272 
Reske et al.

Figure 3-figure supplement 1. Supplemental ARID1A knockdown differential H3.3 data.

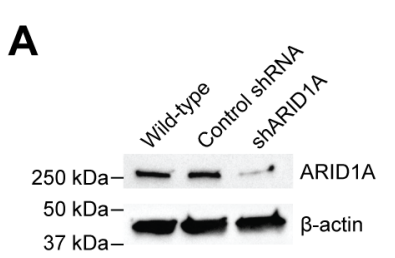

B

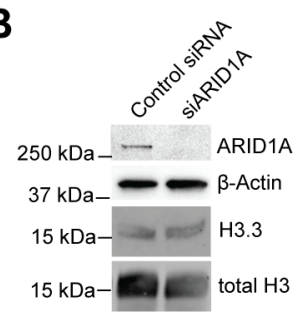

C
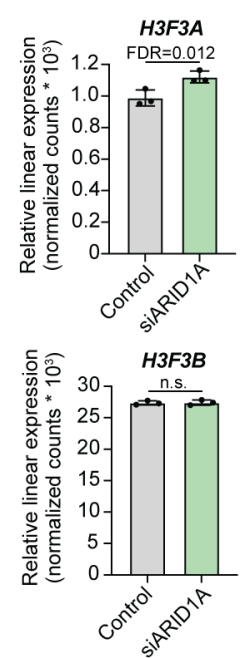

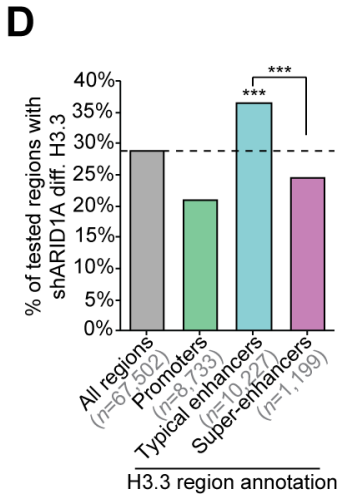

E

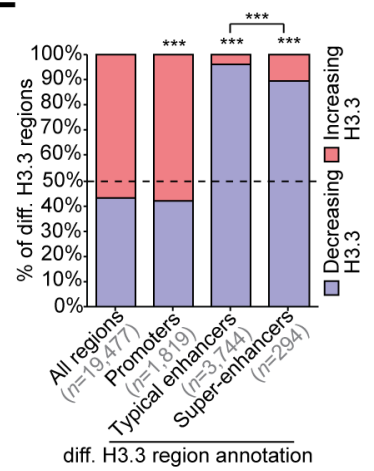

275

276

277

278

279

280

281

282

283

284

285

286

287

288

A, ARID1A immunoblot, compared to $\beta$-actin loading control, in $12 Z$ cells treated with lentiviral shRNA particles to acutely knockdown ARID1A compared to non-targeting control shRNA. Wild-

type condition shown as reference. B, Immunoblot for H3.3 and ARID1A, compared to total H3 and $\beta$-actin loading controls, respectively, in $12 \mathrm{Z}$ cells treated with siRNA to acutely knockdown ARID1A (siARID1A). C, RNA-seq linear gene expression data (normalized counts) for H3.3encoding gene isoforms, $H 3 F 3 A$ and $H 3 F 3 B$, following ARID1A knockdown by siRNA (siARID1A).

D, Enrichment of regions displaying shARID1A significant differential H3.3 abundance among promoters, typical enhancers, and super-enhancers compared to all tested H3.3 regions. Gene promoters are defined as within $3 \mathrm{~kb}$ of a TSS. Enhancers are defined as ATAC + H3K27ac peaks located $>3 \mathrm{~kb}$ from a TSS. Super-enhancers were further distinguished from typical enhancers by ROSE. Statistics are hypergeometric enrichment and pairwise two-tailed Fisher's exact test. E, Distribution of significantly increasing vs. decreasing genomic H3.3 with ARID1A knockdown among the classes described in C. Statistics are hypergeometric enrichment and pairwise two-tailed Fisher's exact test. 
bioRxiv preprint doi: https://doi.org/10.1101/2022.02.27.482165; this version posted February 28, 2022. The copyright holder for this preprint (which was not certified by peer review) is the author/funder, who has granted bioRxiv a license to display the preprint in perpetuity. It is made available under aCC-BY 4.0 International license.

Reske et al.
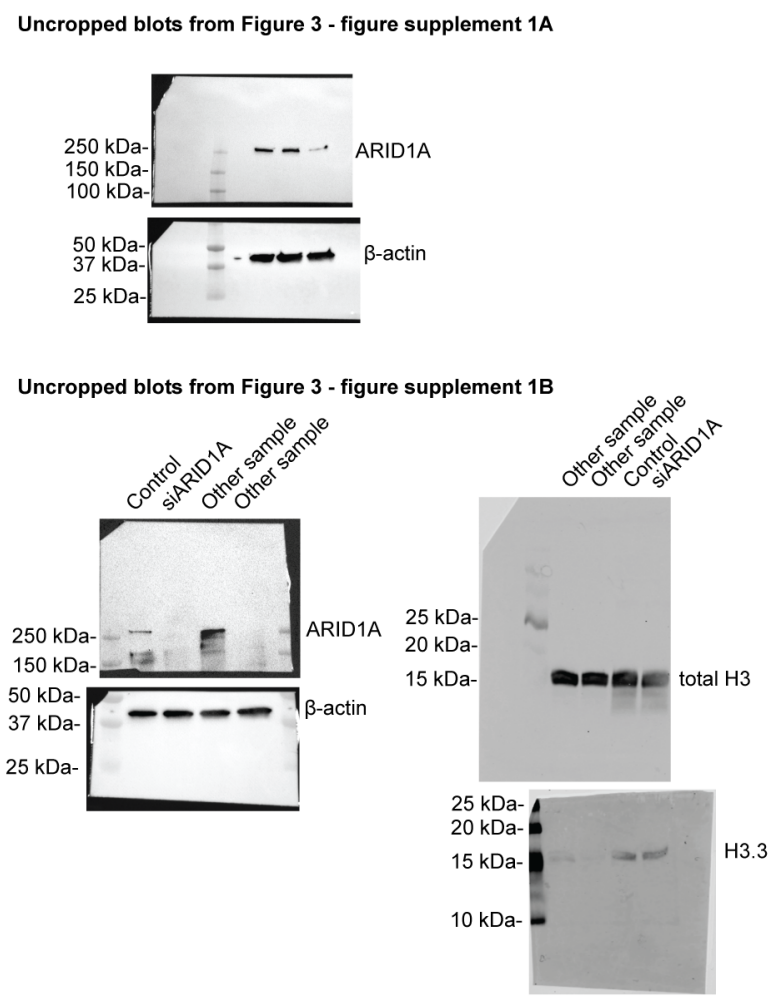
Reske et al.

\section{H3.3 depletion phenocopies transcriptional effects of ARID1A loss.}

We next sought to determine the transcriptional consequences of H3.3 loss in endometrial roles in transcriptional regulation of endometrial epithelial cells. ARID1A loss, we observed significant overlap, with 682 shared dysregulated genes (Figure 4F).

311 These 682 genes were then grouped by direction of change (upregulated vs. downregulated) to

312 identify genes with the same or different expression patterns following ARID1A vs. H3.3 loss. A

313 significant association was observed between the effects of H3.3 and ARID1A loss indicating 314 shared transcriptional consequences (Figure 4G). Gene expression changes also positively 315 correlated transcriptome-wide (Figure 4H). Intriguingly, the 682 genes affected by loss of H3.3 316 and ARID1A were more likely to be repressed by H3.3 (Figure 4I). 196 genes were identified as 
Reske et al.

317 mutually repressed by both ARID1A and H3.3, including PLAU, ADAMTS15, C1S, CD82, CCL2, 318 and CLSTN2 (Figure 4J). In agreement with differential H3.3 patterns, these 196 co-repressed

319 genes were enriched for similar gene sets as observed among the shARID1A direct decreasing

320 promoter H3.3 gene set, including EMT, TNF $\alpha$ signaling, estrogen response, apoptosis, adhesion,

321 migration, extracellular matrix, and collagens (Figure 4K). Altogether, these data suggest that

322 ARID1A and H3.3 co-regulate similar target genes in endometrial epithelial cells. At the chromatin

323 level, depletion or destabilization of H3.3 as a result of ARID1A loss may lead to the upregulation

324 of a physiologically relevant set of EMT and invasion genes. 
bioRxiv preprint doi: https://doi.org/10.1101/2022.02.27.482165; this version posted February 28, 2022. The copyright holder for this preprint (which was not certified by peer review) is the author/funder, who has granted bioRxiv a license to display the preprint in perpetuity. It is made available under aCC-BY 4.0 International license.

Reske et al.

Figure 4. Transcriptional effects of H3.3 depletion and overlap with ARID1A.

A

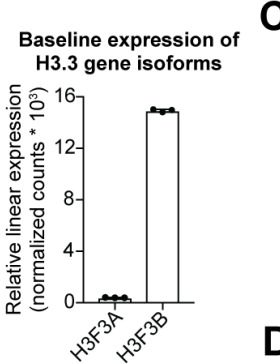

B

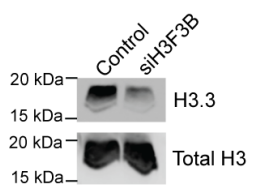

$\mathbf{F}$

DGE overlap (FDR<0.001)

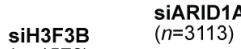

$(n=1578)$

$896 \quad 682 \quad 2,430$

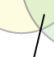

Overlap enrichr

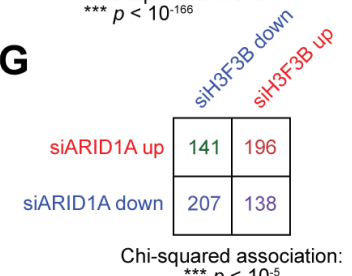

Chi-squared association

D
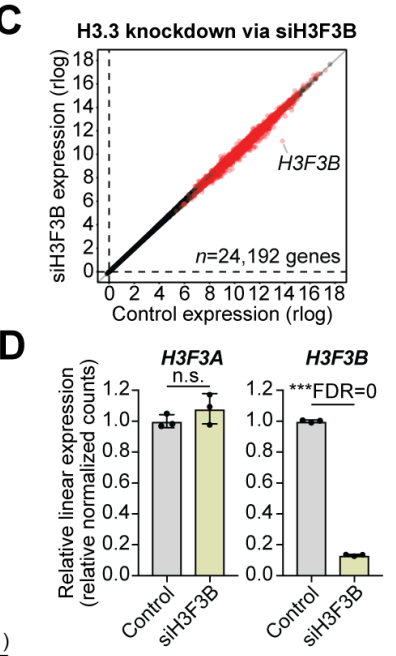

H $\quad r=0.059 ; * * * x<10.18$

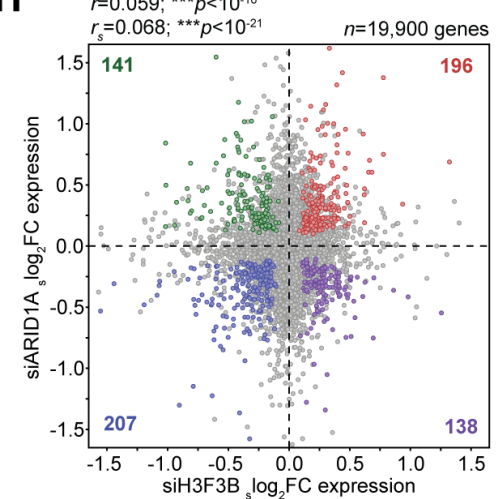

E

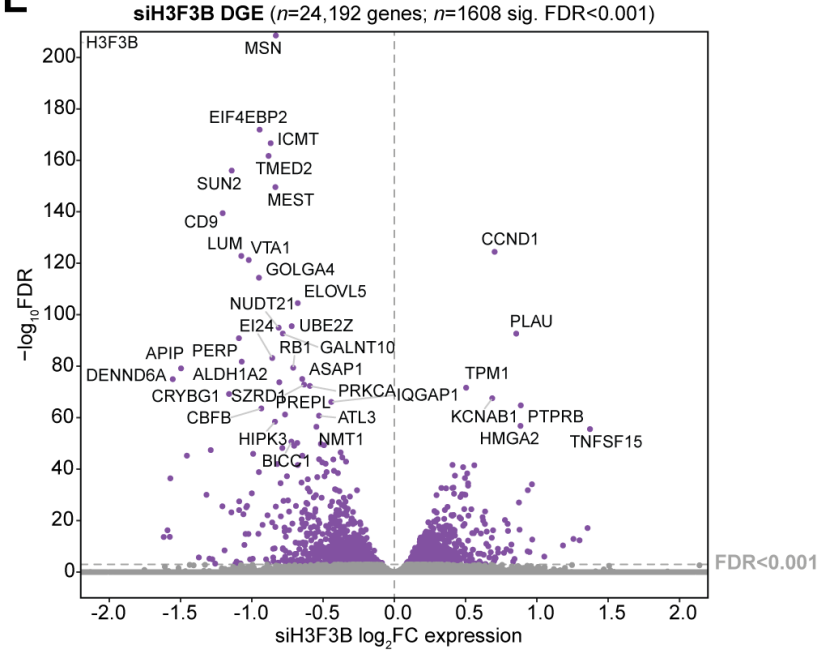

I

Proportion of $\mathrm{siH} 3 \mathrm{~F} 3 \mathrm{~B}$ DE genes

$0 \% \quad 20 \% \quad 40 \% 60 \%$ 80\% $100 \%$

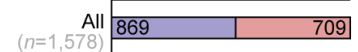

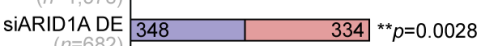

$(n=682)$

$\square$ Downregulated $\square$ Upregulated

K ARID1A-H3.3 mutually repressed genes $(n=196)$ Enrichment (- $\log _{10}$ FDR)

Epithelial-mesenchymal transition 22 Estrogen respons Apoptosis
A KRAS signaling up

Unfolded protein response Hypoxia
UV response (up) Biological adhesion Cell migration 46 Locomotion
development Circulatory system development capsulating structure organization cellular component movement Cell population proliferation Vasculature development

Regulation of cell differentiation

External encapsulating structure 23

Ex-containing extracellular matrix

Endoplasmic reticulum lumen

Side of membrane
Endoplasmic reticulum

External side of plasma membrane Cof plasma membrane
Collagen trimer Anchoring junction 23 Complex of collagen trimers Extracellular matrix organization
Signaling by PDGF Collagen biosynthesis and modifying enzymes Collagen formation ECM proteoglycans Integrin cell surface interactions

Collagen chain trimerization Assembly of collagen fibrils and other Focal adhesion 12
Fon p53 signaling pathway Pathways in cancer Small cell lung cance
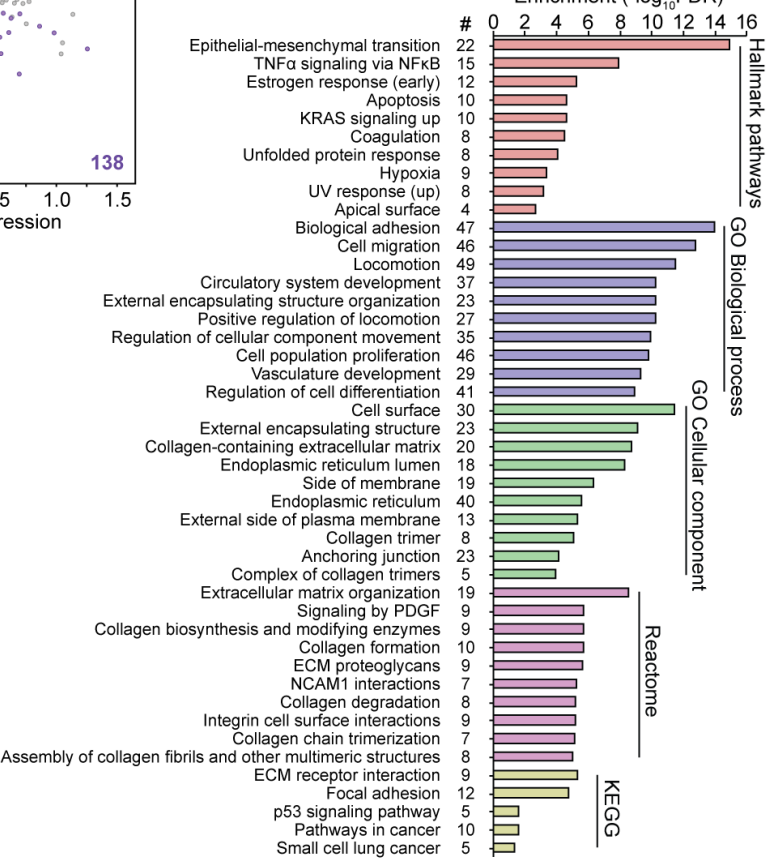

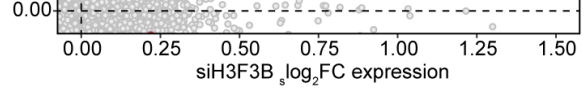

328 A, Baseline relative linear expression of $H 3 F 3 A(H 3-3 A)$ and $H 3 F 3 B(H 3-3 B)$ gene isoforms

329 encoding H3.3, as measured by RNA-seq. B, Western blot for H3.3 and total H3 in control vs. 
Reske et al.

331 knockdown via siH3F3B treatment. Red dots represent significant DE genes (DESeq2, FDR < 0.001). D, Relative linear expression of $H 3 F 3 A$ and $H 3 F 3 B$ by RNA-seq in control and siH3F3B

333 cells. E, Volcano plot depicting siH3F3B vs. control differential gene expression (DGE). Top

334 significant genes are labeled. F, Significant overlap in DE genes following H3.3 knockdown 335 (siH3F3B) vs. ARID1A knockdown (siARID1A). Statistic is hypergeometric enrichment. G, 336 Directional segregation of siH3F3B/siARID1A overlapping DE genes. A positive association is 337 observed by Chi-squared test, i.e., genes are more likely to be upregulated or downregulated in 338 both conditions as opposed to antagonistic regulation. H, Scatter plot of siH3F3B vs. siARID1A 339 expression $\log _{2} \mathrm{FC}$ (with shrinkage correction) for all 19,900 transcriptome-wide commonly 340 detected genes. Statistic is pearson $(r)$ and Spearman $\left(r_{\mathrm{s}}\right)$ correlation coefficients. Colored dots 341 indicate significant DE genes (FDR < 0.001) in both treatment conditions. I, Enrichment for H3.3 342 repression ( $\mathrm{siH} 3 \mathrm{~F} 3 \mathrm{~B}$ upregulation) among siH3F3B genes which are also affected by ARID1A 343 loss (siARID1A). Statistic is hypergeometric enrichment test. J, Scatter plot of 196 shared DE 344 genes upregulated following knockdown of either H3.3 and ARID1A. These genes are mutually 345 repressed by H3.3 and ARID1A. K, Top significant $($ FDR $<0.05)$ enriched gene sets among the 346196 ARID1A-H3.3 mutually repressed genes among various gene set databases. 
Reske et al.

Figure 4-figure supplement 1. H3.3 knockdown functional analysis.
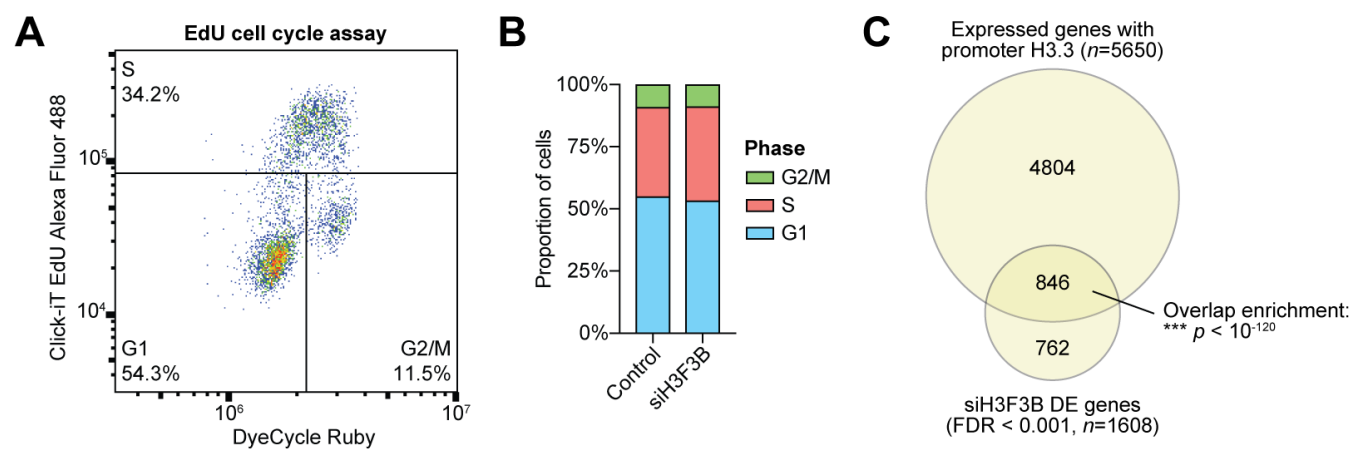

350 A, Representative cell cycle analysis by EdU labeling and DyeCycle ruby staining following 72

351 hours siRNA trasnfection. B, Quantification of cell cycle phases (G1, S, and G2/M) in control non-

352 targeting siRNA treated and siH3F3B (H3.3 knockdown) treated $12 \mathrm{Z}$ cells. C, Euler diagram

353 displaying overlap of genes with detected promoter H3.3 by ChIP-seq $(M A C S 2$, FDR $<0.05)$ and

354 genes with altered expression following H3.3 knockdown by RNA-seq (DESeq2, FDR < 0.001).

355 Statistic is hypergeometric enrichment.

Figure 4-figure supplement 2. Uncropped Western blots from Figure 4.

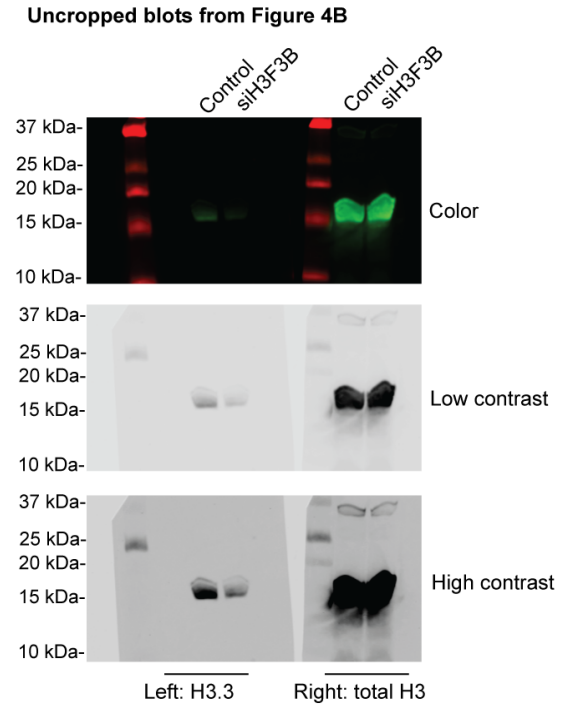

359 Color image is presented for molecular weight marker reference. 
Reske et al.

\section{ARID1A interacts with CHD4 to co-regulate H3.3 with ZMYND8.}

While our data implicate H3.3 in the ARID1A mutant endometrium, few reports have linked SWI/SNF activity to H3.3 containing nucleosomes (Gehre et al., 2020; Pillidge \& Bray, 2019). To gain insight into possible chromatin regulators mediating H3.3 regulation by ARID1A, we used the ReMap2020 database of 165 million peak regions extracted from genome-wide binding assays (Cheneby et al., 2020). For all 1135 transcriptional regulators included in this database, we calculated genome-wide associations for each set of factor peaks with H3.3-marked (H3.3+) vs. H3.3-absent (H3.3-) ARID1A binding. This analysis revealed that two zinc finger MYND-type proteins, ZMYND11 (BS69) and ZMYND8 (PRKCBP1, RACK7), were among the top co-regulators associated with H3.3+ ARID1A chromatin binding (Figure 5A), suggesting that H3.3 regulation by ARID1A may be mediated by these co-regulators. ZMYND11 and ZMYND8 are multivalent chromatin readers that are suggested to function as interfaces between histones and

372 other chromatin regulator complexes like remodelers, writers, and erasers (Guo et al., 2014; 373 Savitsky et al., 2016). Both proteins interact with H3/H4 acetylated tails through bromodomains 374 and may show specificity toward or against H3.3-containing nucleosomes (Adhikary et al., 2016;

375 Guo et al., 2014; N. Li et al., 2016; Wen et al., 2014). Numerous studies have shown ZMYND8 376 interacts with and can recruit the SWI/SNF-like repressive NuRD (Mi2- $\beta$ ) chromatin remodeler 377 complex (Adhikary et al., 2016; Gong et al., 2015; Savitsky et al., 2016; Spruijt et al., 2016), which contains HDACs and interacts with H3.3 (Kraushaar et al., 2018). Like ARID1A, ZMYND8 has

379 also been shown to suppress super-enhancer hyperactivation (Shen et al., 2016), and, more recently, ZMYND8 and ARID1A were identified in the same screen as key chromatin regulators of EMT (Serresi et al., 2021). Therefore, we investigated the potential roles of ZMYND8 and

382 possible co-factors as mediators of the observed ARID1A-H3.3 co-regulation (Figure 5B). 
Reske et al.

ARID1A co-immunoprecipitation (co-IP) using an anti-ARID1A antibody was first used to detect physical nuclear interactions with ZMYND8. We previously confirmed the specificity of the anti-ARID1A antibody by co-immunoprecipitation followed by mass spectrometry (Wilson et al., 2019). While ZMYND8 was not detected in the ARID1A pulldown following high salt washes (300 $\mathrm{mM} \mathrm{KCl),} \mathrm{the} \mathrm{NuRD} \mathrm{catalytic} \mathrm{subunit} \mathrm{CHD4} \mathrm{was} \mathrm{evident} \mathrm{(Figure} \mathrm{5C).} \mathrm{We} \mathrm{then} \mathrm{hypothesized}$ that CHD4-NuRD may serve as an interface between ARID1A and ZMYND8. A reciprocal CHD4 co-IP confirmed nuclear interactions with both ARID1A and ZMYND8 (Figure 5D). To further support that ARID1A, ZMYND8, and CHD4 are found in high molecular weight nuclear complexes of similar size, glycerol gradient sedimentation was performed. Native fractions were observed that included ZMYND8 and members of both SWI/SNF and NuRD (Figure 5E). These data suggest that physical interactions between ARID1A and CHD4 may regulate H3.3 chromatin with support from ZMYND8.

We then examined genome-wide chromatin regulation by CHD4 and ZMYND8 in relation to ARID1A and H3.3. Genome-wide binding profiles of CHD4 and ZMYND8 were measured by ChIP-seq. Roughly 2000 genomic regions were identified with H3.3 and all three chromatin 398 regulators co-localized (Figure 5F-G). Across all H3.3 peaks genome-wide, ARID1A binding was most strongly enriched at ZMYND8-CHD4 co-bound sites compared to sites occupied by either

400 CHD4 or ZMYND8 alone (Figure 5H). Notably, when CHD4 was absent, ARID1A binding at 401 H3.3 sites did not correlate with the presence of ZMYND8 (Figure 5H). These data suggest that 402 CHD4 may be primarily responsible for ARID1A recruitment to H3.3 chromatin. We further 403 investigated ARID1A binding and H3.3 abundance across H3.3 peaks segregated by the presence 404 of CHD4/ZMYND8. ARID1A binding was again strongest at H3.3 peaks co-bound by CHD4 as 405 opposed to those without CHD4 (Figure 5I). H3.3 abundance was similarly highest at CHD4- 
Reske et al.

406 bound peaks, although CHD4+ZMYND8 peaks showed the overall highest H3.3 levels (Figure 407 5I). With respect to H3.3 regions dependent on ARID1A chromatin interactions, we observed that 408 baseline H3.3 levels were significantly higher at regions that decreased in H3.3 following ARID1A 409 knockdown if they were co-occupied by CHD4 or ZMYND8, but this was not observed at regions 410 that gained H3.3 following ARID1A loss (Figure 5J). Intriguingly, we observed that genome-wide 411 H3.3 regions directly maintained by ARID1A chromatin interactions are associated with CHD4

412 but not ZMYND8 (Figure 5K). Moreover, genome-wide regions that lose H3.3 following ARID1A

413 loss due to disrupted ARID1A chromatin interactions tend to have higher baseline levels of 414 ARID1A, CHD4, and H3.3, but lower levels of ZMYND8 in comparison to stable H3.3 regions 415 (Figure 5L). Altogether, these results suggest CHD4 and ZMYND8 are associated with ARID1A416 H3.3 co-regulation. 
bioRxiv preprint doi: https://doi.org/10.1101/2022.02.27.482165; this version posted February 28, 2022. The copyright holder for this preprint (which was not certified by peer review) is the author/funder, who has granted bioRxiv a license to display the preprint in perpetuity. It is made available under aCC-BY 4.0 International license.

Reske et al.

A

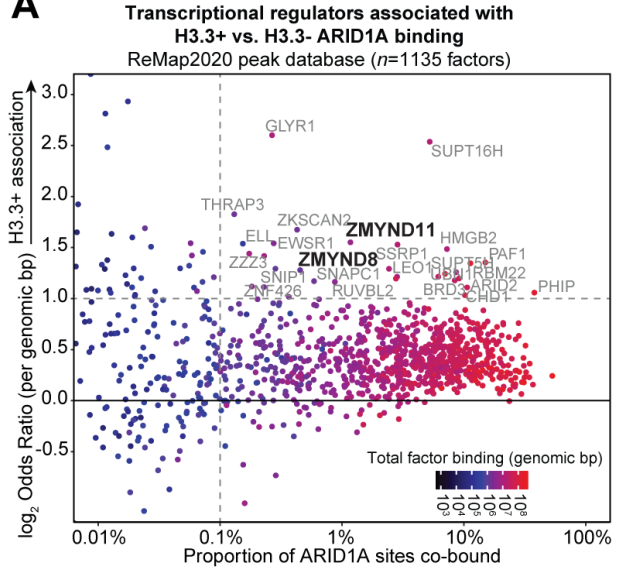

C

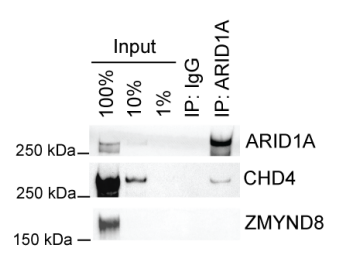

D

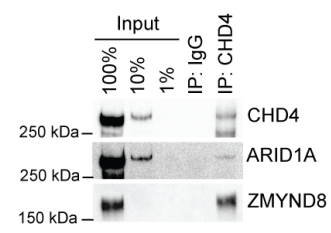

E

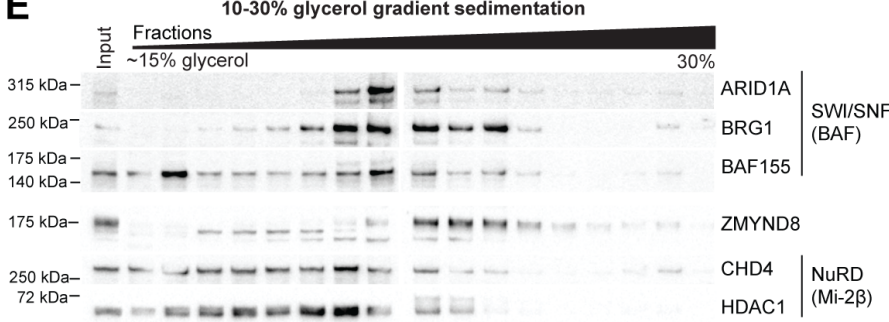

Potential interacting native complexes

B

$\mathbf{F}$
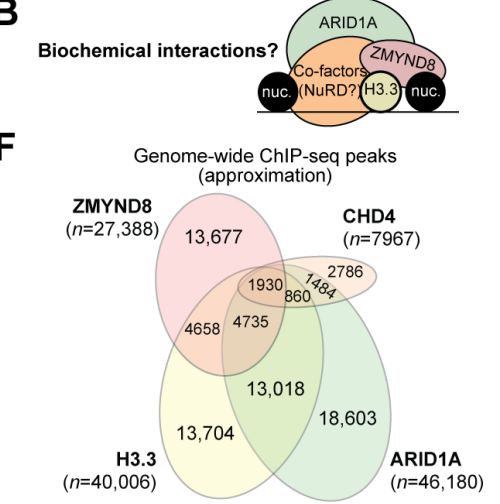

I
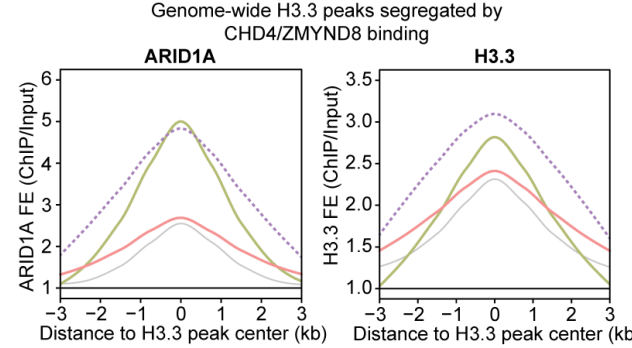

$$
\text { H3.3 peaks: }- \text { CHD4+ZMYND8 - ZMYND8-only }
$$

K

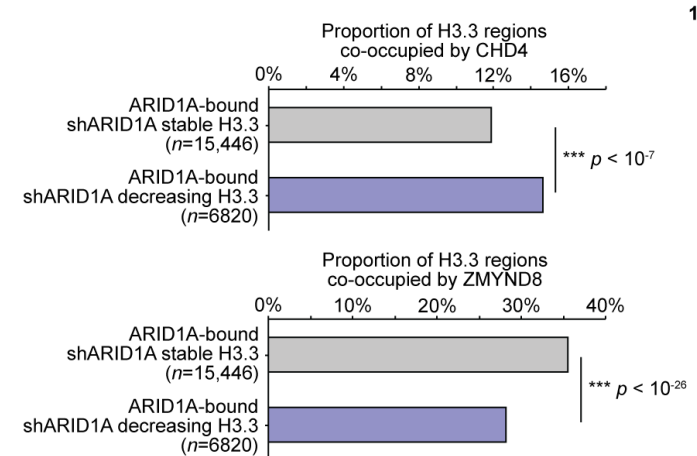

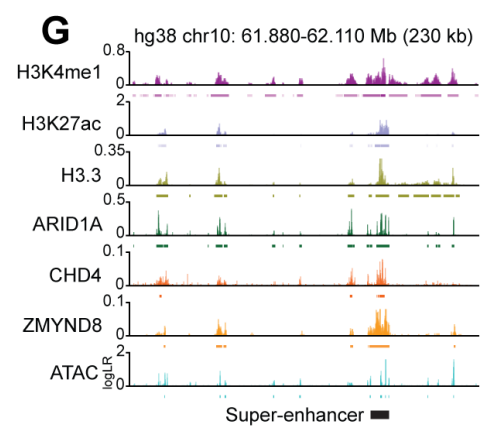

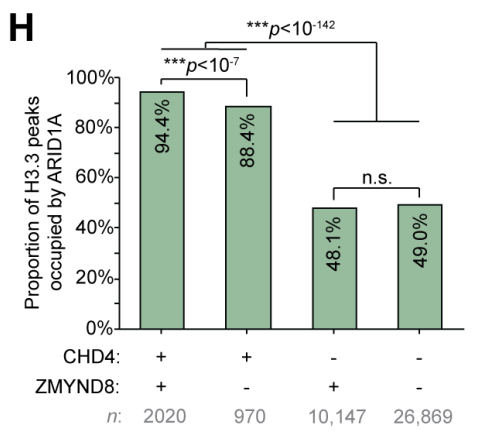

J H3.3 levels at ARID1A directly regulated H3.3+ regions co-occupied by CHD4/ZMYND8

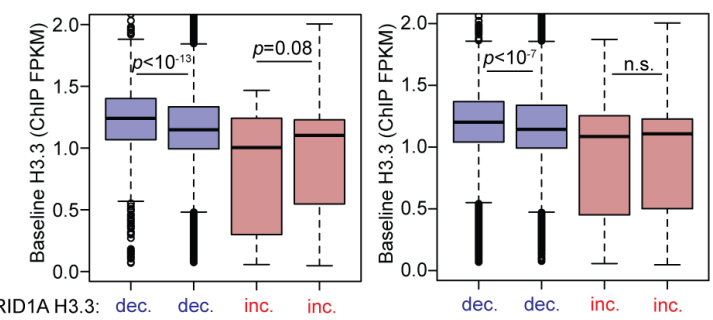

CHD4: +

$998 \quad 5822 \quad 42 \quad 333$

ZMYND8: +

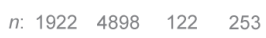

L - ARID1A-bound, shARID1A decreasing $\mathrm{H} 3.3(n=6820)$
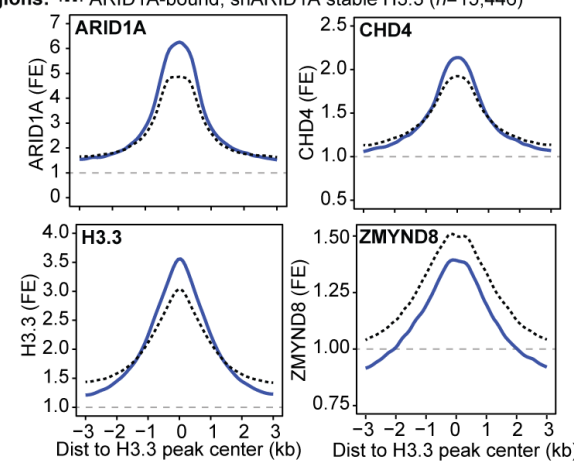
Reske et al.

421 A, Genome-wide associations between ARID1A binding at H3.3+ vs. H3.3- regions for all 1135

422 transcriptional regulator peak sets included in the ReMap2020 peak database. Labeled factors

423 exhibit an H3.3+ ARID1 A binding association with genomic odds ratio $>2$ and overlap with $>0.1 \%$

424 of ARID1A binding sites. ZMYND11 and ZMYND8 (bolded) are two of the top factors most

425 associated with H3.3+ ARID1A binding. B, Chromatin model schematic depicting hypothesized

426 relationship between ARID1A-SWI/SNF and ZMYND8 co-regulation of H3.3, possibly mediated

427 by co-factors. C, ARID1A co-immunoprecipitation detecting physical interaction with NuRD

428 catalytic subunit CHD4, but not ZMYND8. D, CHD4 co-immunoprecipitation detecting physical

429 interactions with both ARID1A and ZMYND8. E, 10-30\% glycerol gradient sedimentation and

430 immunoblotting for SWI/SNF, NuRD, and ZMYND8. Relative fractions display native protein

431 complexes transitioning from low molecular weight (left) to high molecular weight (right).

432 Underlined fractions highlight potential interacting native complexes containing ZMYND8 and

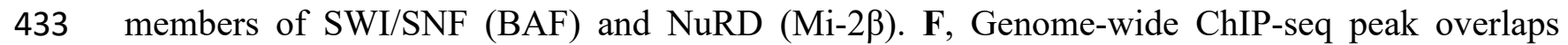

434 between ARID1A, CHD4, ZMYND8, and H3.3. Peak numbers within the Euler diagram are

435 approximations and not mutually exclusive due to varying peak sizes. G, Example locus on

436 chromosome 10 displaying ARID1A, CHD4, ZMYND8, and H3.3 co-regulation. H, Enrichment

437 for ARID1A co-regulation of H3.3 peaks bound by CHD4 and/or ZMYND8. Statistic is two-tailed

438 Fisher's exact test. I, Average ChIP-seq signal density histograms for ARID1A (left) and H3.3

439 (right) at H3.3 peaks bound by CHD4 and/or ZMYND8. J, H3.3 abundance (ChIP FPKM) at

440 ARID1A-bound shARID1A differential H3.3 regions co-bound by CHD4 or ZMYND8. Statistic

441 is two-tailed, unpaired Wilcoxon's test. K, Positive association between CHD4 binding (top) and

442 negative association between ZMYND8 binding (bottom) and ARID1A direct maintenance of

443 H3.3 chromatin, genome-wide. Statistic is two-tailed Fisher's exact test. L, Average ChIP-seq 
bioRxiv preprint doi: https://doi.org/10.1101/2022 02.27 .482165 . this version posted February 28,2022 . The copyright holder for this preprint (which was not certified by peer review) is the author/funder, who has granted bioRxiv a license to display the preprint in perpetuity. It is made available under aCC-BY 4.0 International license.

Reske et al.

444 signal density histograms for ARID1A, H3.3, CHD4, and ZMYND8 across ARID1A-bound H3.3

445 regions that decreased or were stable with shARID1A.

Figure 5-figure supplement 1. Uncropped Western blots from Figure 5.
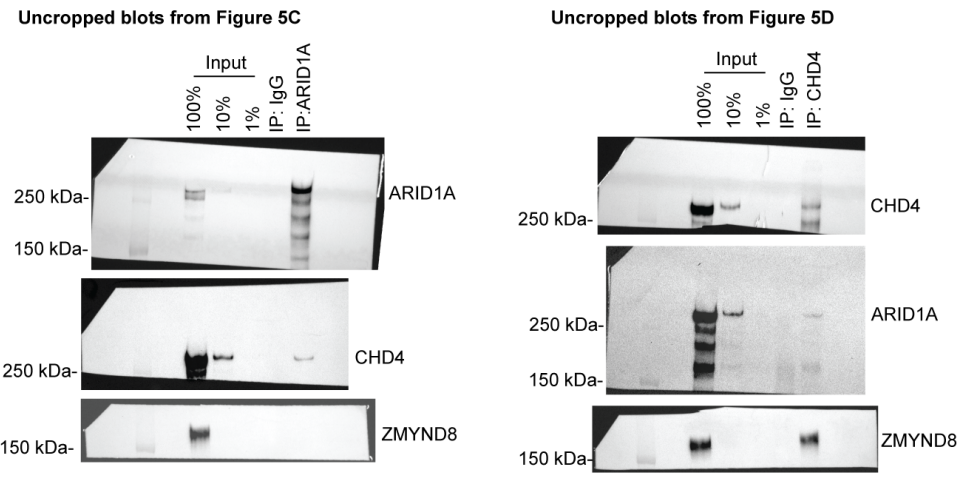

Uncropped blots from Figure $5 E$
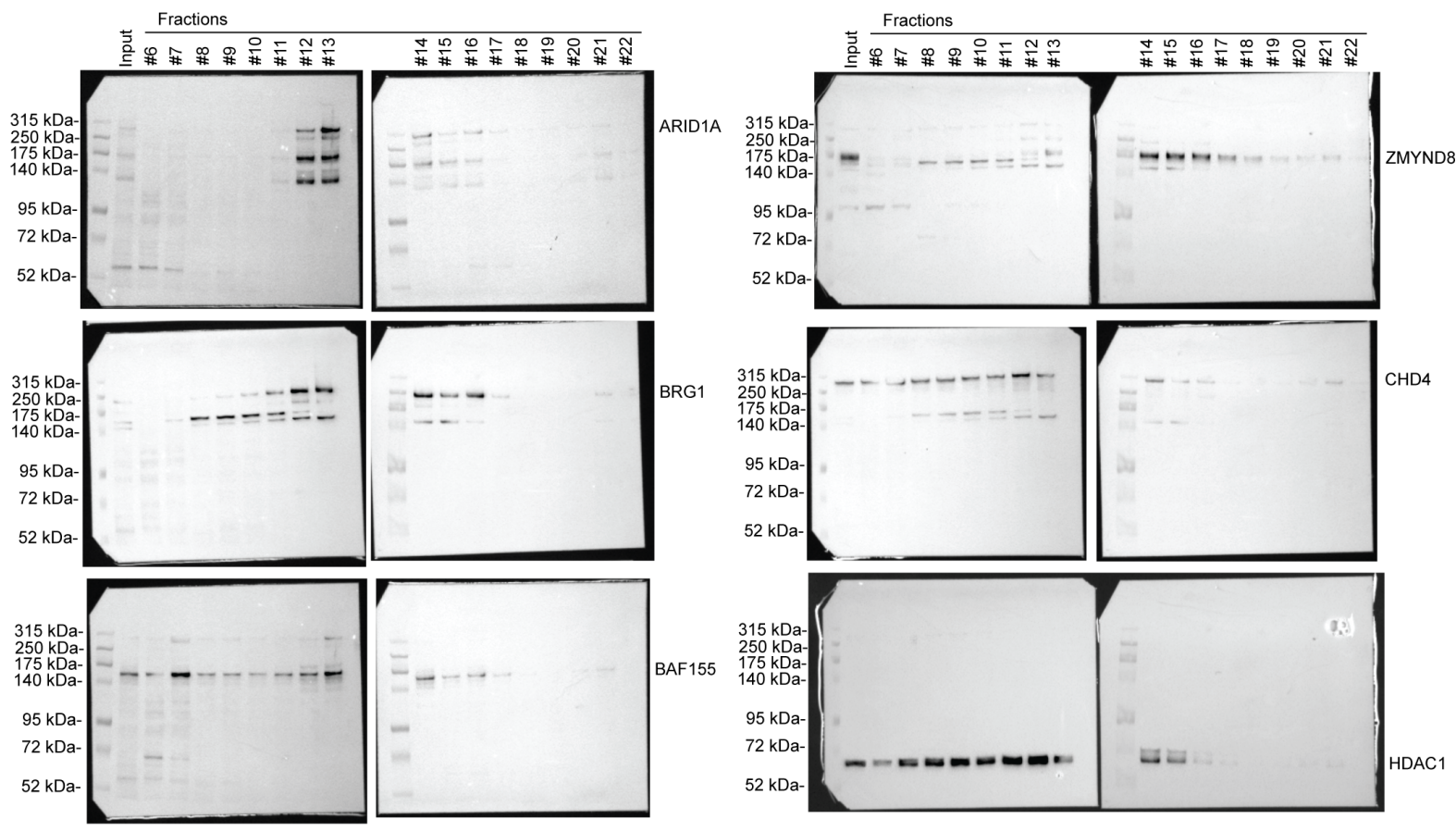
Reske et al.

\section{ARID1A-CHD4-ZMYND8 suppress hyperactivation of H3.3-marked super-enhancers.}

H3.3 may be chromatin context-specific and occur at subsets of regulatory regions bound by ARID1A-CHD4-ZMYND8. While ARID1A loss causes widespread H3.3 reduction in chromatin, we observed that ARID1A-H3.3 co-regulation is most frequent at active enhancer and super-

455 enhancer chromatin states. As such, we next examined ARID1A-CHD4-ZMYND8 co-regulation

456 of H3.3 at enhancers. At ARID1A-bound active enhancers, defined as accessible (ATAC+)

$457 \mathrm{H} 3 \mathrm{~K} 27 \mathrm{ac}$ peaks located $>3 \mathrm{~kb}$ from an annotated TSS (Figure 6A), ZMYND8 binding is associated 458 with presence of CHD4, as expected (Figure 6B). Moreover, ZMYND8 binding was detected at $84.6 \%$ of ARID1A+CHD4 super-enhancers $(n=507)$ as opposed to $63.2 \%$ of ARID1A+CHD4 460 typical enhancers $(n=2282)$ (Figure 6B). Importantly, ARID1A, CHD4, and ZMYND8 co461 binding at active enhancers is associated with presence of $\mathrm{H} 3.3$, and this association is greater at 462 super-enhancers (Figure 6C). We previously observed that ARID1A suppresses H3K27463 hyperacetylation at a subset of active super-enhancers (Wilson et al., 2020). ARID1A and CHD4 464 binding levels are not substantially different at suppressed super-enhancers that become 465 hyperacetylated following ARID1A loss vs. those with stable acetylation (Figure 6D). Strikingly, 466 ZMYND8 binding and H3.3 abundance are significantly higher at suppressed super-enhancers that 467 become hyperacetylated (Figure 6D). These data indicate that ZMYND8 is associated with CHD4 468 and ARID1A most frequently at active H3.3-marked super-enhancers that are suppressed by 469 ARID1A. 
bioRxiv preprint doi: https://doi.org/10.1101/2022.02.27.482165; this version posted February 28, 2022. The copyright holder for this preprint (which was not certified by peer review) is the author/funder, who has granted bioRxiv a license to display the preprint in perpetuity. It is made available under aCC-BY 4.0 International license.

Reske et al.

Figure 6. H3.3 enhancer regulation by ARID1A, CHD4, and ZMYND8.

A

472

473 A, Heatmap of chromatin features at 15,925 active typical enhancers and 1374 distal super-

474 enhancer constituents (H3K27ac peaks co-marked by ATAC) segregated by ARID1A \pm CHD4 \pm

475 ZMYND8 binding. Enhancers are centered on the H3K27ac peak, and signal is displayed as

476 indicated for the flanking $5 \mathrm{~kb}$ in either direction. B, ZMYND8 binding detection at ARID1A-

477 bound typical and super-enhancers with or without CHD4 co-binding. Statistic is two-tailed

478 Fisher's exact test. C, Association between ARID1A+CHD4+ZMYND8 co-binding at enhancers 
Reske et al.

479 and presence of H3.3. H3.3 + super-enhancers show the most frequent co-binding. Statistic is two-

480 tailed Fisher's exact test. D, Chromatin features at active super-enhancer constituents segregated

481 by ARID1A loss-driven H3K27-acetylation dynamics: hyper-acetylated, de-acetylated, or stably

482 acetylated. Left, average ChIP-seq signal density histograms across enhancer classes. Right, violin

483 plots quantifying signal (ChIP/input fold-enrichment) across enhancer classes. Statistic is two-

484 tailed, unpaired Wilcoxon's test. 
Reske et al.

\section{ZMYND8 specifies ARID1A-CHD4 chromatin repression toward H4K16ac.}

Our data indicate the ZMYND8 module appears to be associated with repressive chromatin

are a plausible mechanism through its BRD, PWWP, and PHD domains, which interact with

ZMYND8 bromodomain was recently described to interact with acetylated H4 tails and recruit

492 CHD4 to repress transcription following DNA damage (Gong et al., 2015). However, NuRD also

493 interacts with other histone substrates such as H2A.Z, which has been linked to H3.3-mediated

transcriptional poising (Chen et al., 2013). Of further relevance, P300 was recently shown to

acetylate H2A.Z when stimulated by recognition of $\mathrm{H} 4$ acetylated residues through its

496 bromodomain (Colino-Sanguino et al., 2019). To better resolve our model of ARID1A-CHD4-

497 ZMYND8-mediated H3.3 chromatin repression, we generated a more comprehensive genome-

498 wide chromatin state model that contains 5 additional features related to reported ZMYND8 and

499 NuRD activity that were profiled both before and after ARID1A knockdown: H3.3, pan-acetyl-H4

500 (K5/K8/K12/K16, pan-H4ac), H4K16ac, H2A.Z, and acetyl-H2A.Z (K4/K7; H2A.Zac) (Figure

501 7A, Figure 7-figure supplement 1). Since the anti-H2A.Zac antibody used in our assays has not

502 been characterized for specificity towards acetylated H2A.Z containing peptides, we performed a

503 histone peptide array (Rothbart, Krajewski, Strahl, \& Fuchs, 2012) and found that it specifically

504 recognizes H2A.Zac (Figure 7-figure supplement 2). The new 12-feature chromatin state model

505 was quantitatively optimized at 25 chromatin states (see Materials and Methods for details) (Figure

506 7-figure supplement 3). The enhanced resolution of our model revealed new state identities such

507 as $\mathrm{H} 3.3+$ poised bivalent promoters (state 8), H2A.Zac + poised enhancers (state 14), and notably

508 the segregation of upstream active promoter super-enhancers into H4(K16)ac+ (state 2) and 
Reske et al.

negative (state 5) classes (Figure 7A). We next determined states enriched for co-binding of ARID1A-CHD4-ZMYND8 and shARID1A direct decreasing H3.3 (Figure 7B). Both upstream active promoter super-enhancer states (states 2 and 5) showed the strongest enrichment for

512 shARID1A direct decreasing H3.3, while ZMYND8 binding and ARID1A-CHD4-ZMYND8 co-

513 binding was most enriched at the H4(K16)ac+ upstream active promoter super-enhancer class

514 (state 2) (Figure 7B).

We further investigated chromatin state identities at reference annotated gene promoter regions ( \pm 3 kilobases flanking TSS). As expected, the highly active TSS state (state S1) is the most prevalent promoter chromatin state identity at genes transcriptionally regulated by ARID1A (siARID1A DE genes), while upstream active promoter super-enhancer states (states 2 and 5) are relatively rare (Figure $7 \mathrm{C}$, left). However, comparing the promoter chromatin state identities across siARID1A DE vs. stable genes revealed that upstream active promoter super-enhancer states are

521 associated with ARID1A transcriptional regulation (Figure 7C, center). We further segregated 522 promoter chromatin based on genes that are upregulated with siARID1A (i.e. repressed by 523 ARID1A) vs. downregulated with siARID1A (i.e. activated by ARID1A). The H4(K16)ac+ active 524 promoter super-enhancer state marked by high ARID1A-CHD4-ZMYND8 co-binding (state 2)

525 showed stronger enrichment for ARID1A transcriptional repression than those without H4(K16)ac

526 (state 5) (Figure 7C, right). In agreement, we also observed that chromatin accessibility directly 527 repressed by ARID1A is associated with presence of $\mathrm{H} 4$ acetylation (Figure 7-figure supplement 528 4). Promoter super-enhancer $\mathrm{H} 3 \mathrm{~K} 27 \mathrm{ac}$ peaks were next directly segregated by detection of 529 H4K16ac (H4K16ac+, $n=112$; H4K16ac-, $n=115)$. ARID1A binding and H3.3 abundance were 530 not significantly different between H4K16ac stratified super-enhancers, but ZMYND8 binding 531 was stronger at the H4K16ac+ regions, while CHD4 binding was lower at H4K16ac+ regions 
Reske et al.

532 (Figure 7D). Further, a correlation of measured chromatin features across all 227 promoter-

533 proximal super-enhancer constituent enhancers supported that ZMYND8 binding is associated

534 with acetylated H4 marks (Figure 7-figure supplement 5). These analyses collectively suggest

535 that H4(K16)ac marked promoter super-enhancers may recruit repressive ARID1A-CHD4-

536 ZMYND8 complexes to regulate H3.3.

537 To identify genes targeted by the ARID1A-CHD4-ZMYND8 regulation of repressive

538 H3.3, we also used siRNA to deplete CHD4 (siCHD4) and ZMYND8 (siZMYND8) followed by

539 RNA-seq (Figure 7E, Figure 7-figure supplement 6A-F). As expected, we observed enrichment

540 of expression alterations following loss of ZMYND8 and CHD4 among genes with detected

541 promoter binding by each factor (Figure 7-figure supplement 6G-H). To further support whether

542 ZMYND8 is associated with transcriptional repression by ARID1A and H3.3, we investigated

543 ZMYND8-mediated gene expression at siARID1A/siH3F3B DGE directional classes (refer to

544 Figure 4G-H). Indeed, siZMYND8 gene expression alterations were strongly enriched at genes

545 mutually repressed by ARID1A and H3.3 compared to other gene classes, where ZMYND8 also

546 functioned mostly as a repressor (Figure 7-figure supplement 6I).

547 Differential gene expression again revealed highly overlapping genes transcriptionally

548 regulated by ARID1A, CHD4, ZMYND8, and H3.3 (Figure 7-figure supplement 6J-L),

549 including 603 genes affected by each of the four knockdowns (FDR < 0.05) (Figure 7F, Figure

550 7-figure supplement 6L). These included 60 genes mutually repressed by ARID1A, CHD4,

551 ZMYND8, and H3.3 (Figure 7G). These mechanistic co-repressed genes were enriched for EMT,

552 adhesion, development, locomotion, collagens, and extracellular matrix gene sets (Figure 7H).

553 Further, $68 \%$ of these genes were marked by gene body H4K16ac, an enrichment compared to less

554 than half of all expressed genes (Figure 7-figure supplement 7). Two physiologically relevant 
Reske et al.

555 target genes revealed through integrative epigenomic analysis are PLAU and TRIO, both of which

556 are located within broad H4K16ac+ domains and near active H3.3+ super-enhancers co-bound by

557 ARID1A, CHD4, and ZMYND8 (Figure 7I). ARID1A loss leads to decreased promoter H3.3

558 abundance and transcriptional hyperactivation of PLAU and TRIO (Figure 7I). We also observed

559 that co-knockdown of ARID1A and CHD4 led to increased induction of PLAU compared to either

560 knockdown separately (Figure 7-figure supplement 8).

561 
bioRxiv preprint doi: https://doi.org/10.1101/2022.02.27.482165; this version posted February 28, 2022. The copyright holder for this preprint (which was not certified by peer review) is the author/funder, who has granted bioRxiv a license to display the preprint in perpetuity. It is made available under aCC-BY 4.0 International license.

Reske et al.

Figure 7. ZMYND8-mediated chromatin repression is specified by H4(K16)ac.

A 12 feature chromatin state model

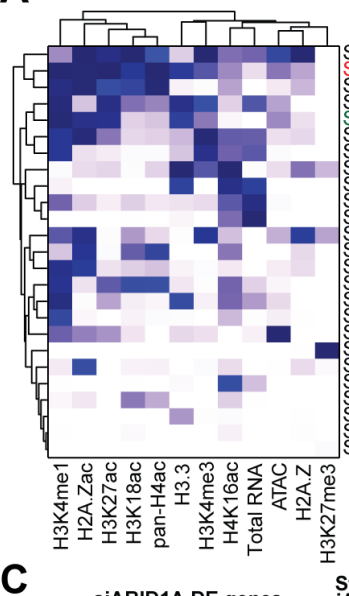

C

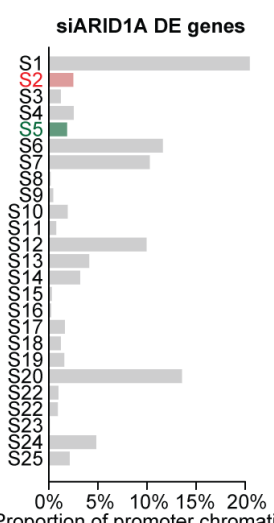

$\begin{array}{rl}0 \% & 5 \% \quad 10 \% 15 \% \quad 20 \% \\ \text { Proportion of promoter chromatin }\end{array}$

E

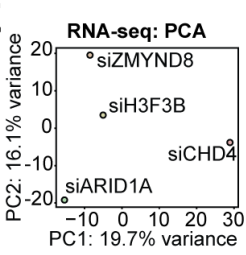

$\mathbf{F}$

States associated with
siARID1A DE (vs. stable)

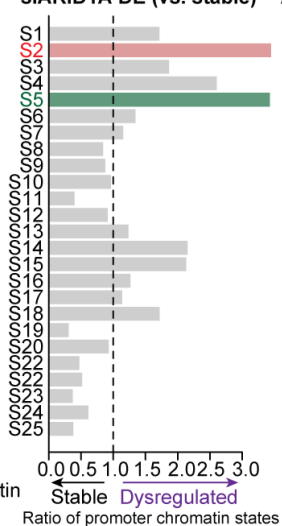

$\mathbf{F}_{\text {FDR }<0.05 \text { DGE intersects }}$ SIH3F3B SIARID1A

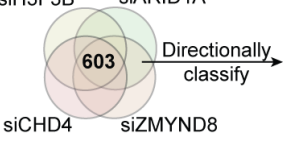

siCHD4 siZMYND8

H ARID1A-CHD4-ZMYND8-H3.3 co-repressed genes $(n=60)$

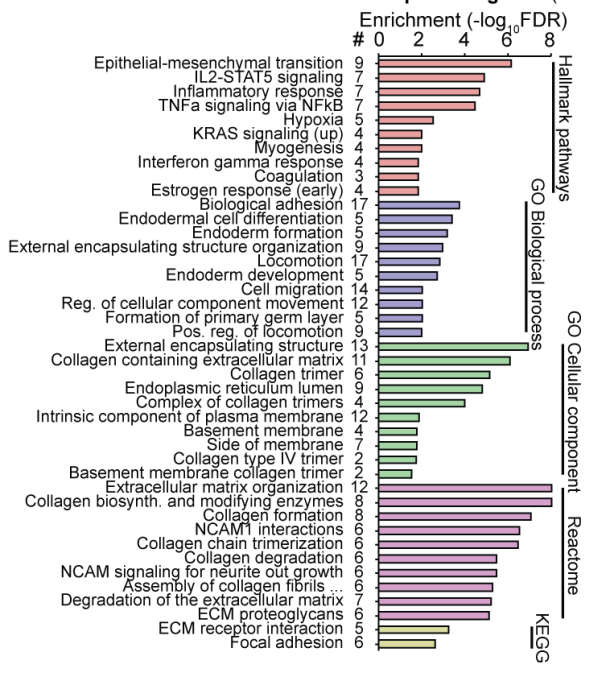
\begin{tabular}{lll} 
Enrichment & $\left(-\log _{60} \mathrm{FDR}\right)$ \\
\hline 0 & 2 & 4
\end{tabular}

563

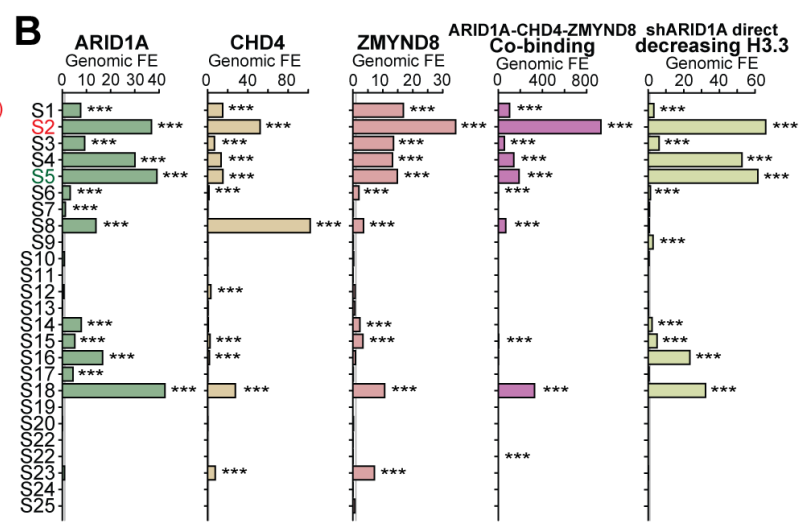

D Chromatin features of H4K16ac+ vs. H4K16ac-
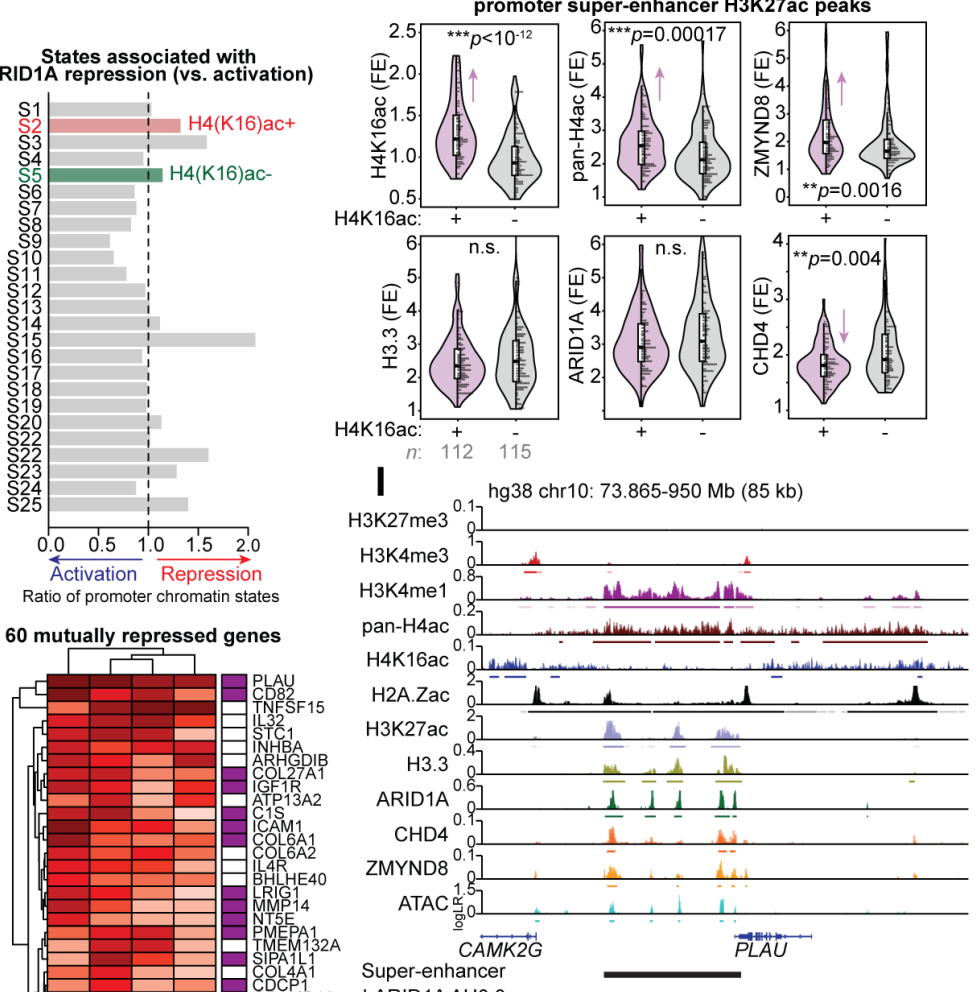

60 mutually repressed genes

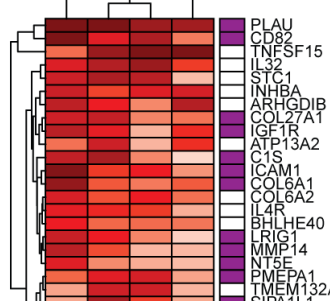

Super-enhancer

shARID1A $\triangle \mathrm{H} 3.3$ hg38 chr5: 13.950-14.550 Mb (600 kb)

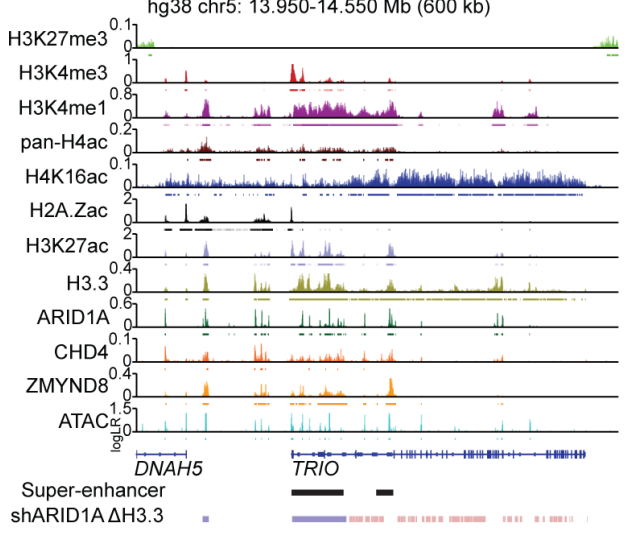


Reske et al.

A, Heatmap of clustered, normalized feature emission probabilities and associated functional annotation of the new 12 feature, genome-wide chromatin 25-state model. States (S_) are labeled based on order of normalized emission probability clustering. See methods for details on optimal model selection. B, Genomic fold-enrichment (FE) for ARID1A, CHD4, ZMYND8, co-binding, and shARID1A direct decreasing H3.3 among the 25 chromatin states. Statistic is hypergeometric enrichment test. C, Modeled chromatin states among reference gene promoter regions $( \pm 3 \mathrm{~kb}$ around annotated TSS). Left, proportion of promoter chromatin for siARID1A DE genes (DESeq2,

571 FDR $<0.0001)$ belonging to each of the 25 states. Center, ratio of promoter chromatin states associated with siARID1A DE genes (FDR < 0.0001) compared to stable genes (FDR > 0.05).

573 Right, ratio of promoter chromatin states associated with ARID1A transcriptional repression (i.e.

574 siARID1A upregulation) compared to activation (i.e. siARID1A downregulation). D, Violin plots 575 quantifying chromatin feature signal at H4K16ac+ (purple) vs. H4K16ac- (gray) promoter super576 enhancer constituent H3K27ac peaks. Statistic is two-tailed, unpaired Wilcoxon's test. E, Principal 577 component analysis (PCA) of RNA-seq expression $\log _{2} \mathrm{FC}$ (shrinkage-corrected) values for 578 siCHD4, siZMYND8, siH3F3B, and siARID1A treatment conditions vs. controls. 1974 genes with $579{ }_{\mathrm{s}} \log _{2} \mathrm{FC}$ variance $>0.1$ were used for PCA. F, Schematic of identifying mechanistic genes co580 repressed by ARID1A, H3.3, CHD4, and ZMYND8, i.e., upregulated (DESeq2, FDR < 0.05) with 581 siARID1A, siH3F3B, siCHD4, and siZMYND8 treatments. G, Clustered heatmap of expression $582 \log _{2} \mathrm{FC}$ values for 60 co-repressed genes upregulated in all 4 knockdown conditions. Rightmost 583 column demarcates presence of H4K16ac peaks over promoter or gene body. H, Top gene sets 584 enriched (hypergeometric enrichment test, FDR $<0.05$ ) among the 60 ARID1A-CHD4585 ZMYND8-H3.3 co-repressed genes from various gene set databases. I, Example target gene loci, 586 PLAU and TRIO, marked by nearby H3.3+ super-enhancers within H4(K16)ac+ domains that are 
bioRxiv preprint doi: https://doi.org/10.1101/2022.02.27.482165; this version posted February 28, 2022. The copyright holder for this preprint (which was not certified by peer review) is the author/funder, who has granted bioRxiv a license to display the preprint in perpetuity. It is made available under aCC-BY 4.0 International license.

Reske et al.

587 co-bound by ARID1A, CHD4, ZMYND8, where ARID1A loss leads to significant depletion of

588 H3.3 (ChIP-seq FDR < 0.05), and knockdown of ARID1A, H3.3, CHD4, or ZMYND8 leads to

589 significant expression upregulation (RNA-seq FDR $<0.05)$.

590 
bioRxiv preprint doi: https://doi.org/10.1101/2022.02.27.482165; this version posted February 28, 2022. The copyright holder for this preprint (which was not certified by peer review) is the author/funder, who has granted bioRxiv a license to display the preprint in perpetuity. It is made available under aCC-BY 4.0 International license.

Reske et al.

Figure 7-figure supplement 1. Additional chromatin features profiled in $12 \mathrm{Z}$ cells.

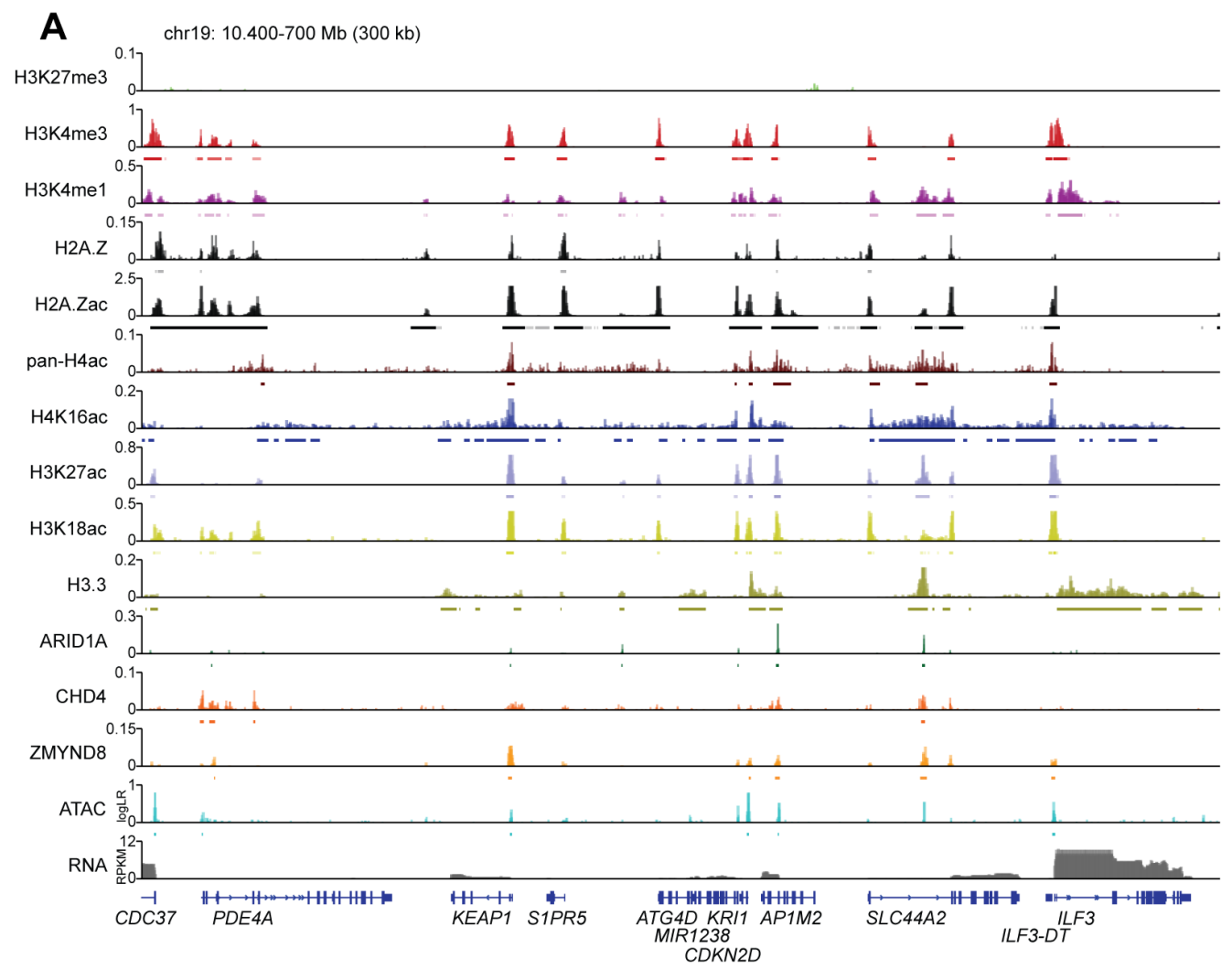

B
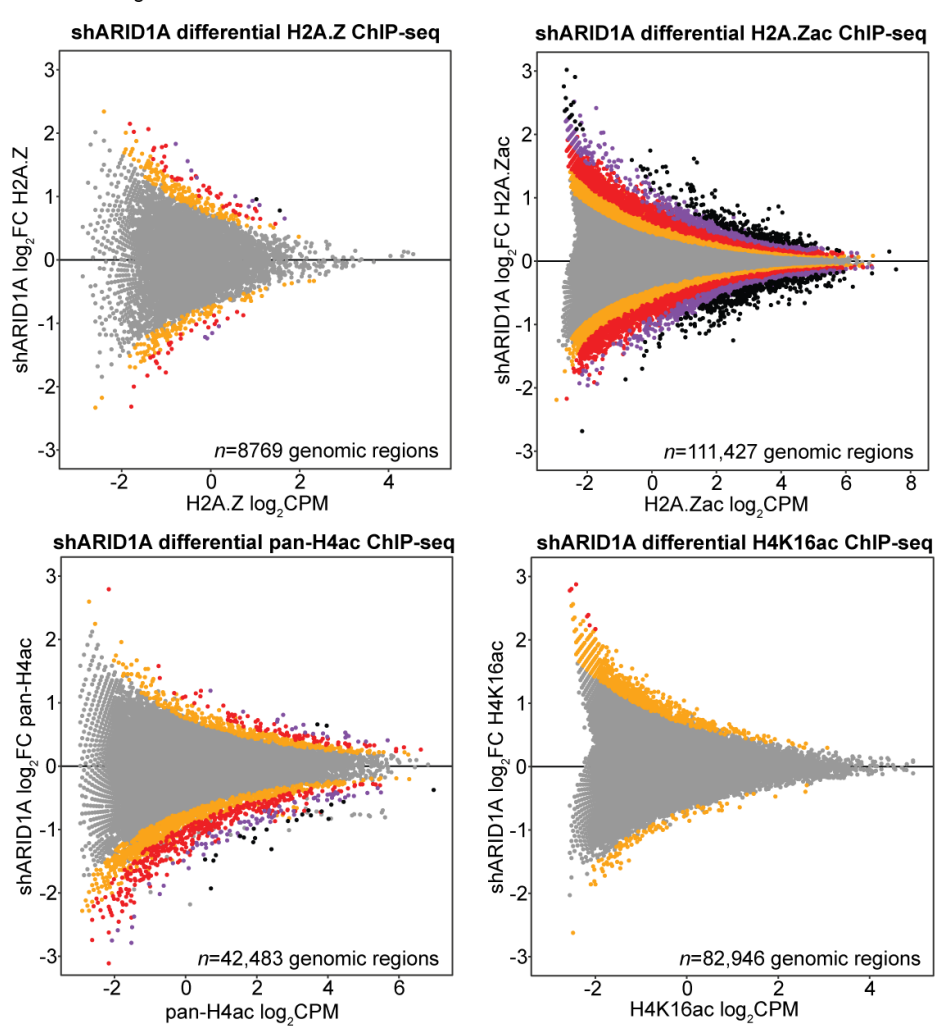
Reske et al.

593 A, Example hg38 locus on chromosome 19 depicting all analyzed chromatin features in this study.

$594 y$-axis is log-likelihood ratio (logLR) of assay signal (compared to input chromatin for ChIP-seq

595 or background genome for ATAC-seq) or RPKM for total RNA. Small bars under tracks indicate

596 significant peak detection by MACS2 (FDR < 0.05). B, MA plots for new shARID1A vs. control

597 differential ChIP-seq experiments: H2A.Z, H2A.Zac (K4/K7), pan-H4ac (K5/K8/K12/K16), and

598 H4K16ac. Genomic regions are colored by significance (csaw/edgeR).

599

Figure 7-figure supplement 2. Peptide specificity of anti-acetyl-H2A.Z (K4/K7).

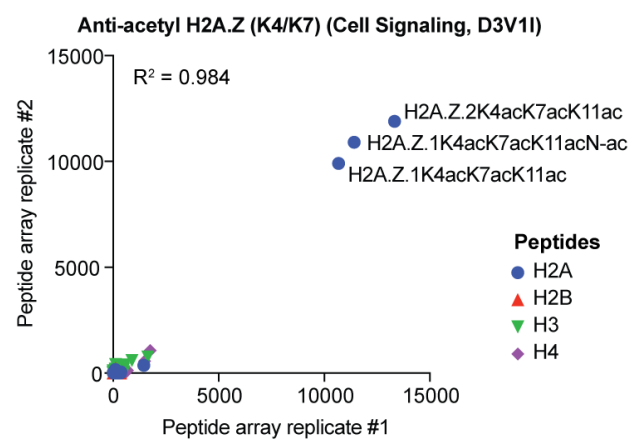

602 Hybridizaiton results for two independent histone peptide microarray replicates probed with anti-

603 acetyl-H2A.Z (H2A.Zac) (Cell Signaling, D3V1I), demonstrating clear specificity for acetylated

604 H2A.Z peptides. The three dominantly detected H2A.Zac peptides are labeled.

605 
bioRxiv preprint doi: https://doi.org/10.1101/2022.02.27.482165; this version posted February 28, 2022. The copyright holder for this preprint (which was not certified by peer review) is the author/funder, who has granted bioRxiv a license to display the preprint in perpetuity. It is made available under aCC-BY 4.0 International license.

Reske et al.

Figure 7-figure supplement 3. ChromHMM model optimization.
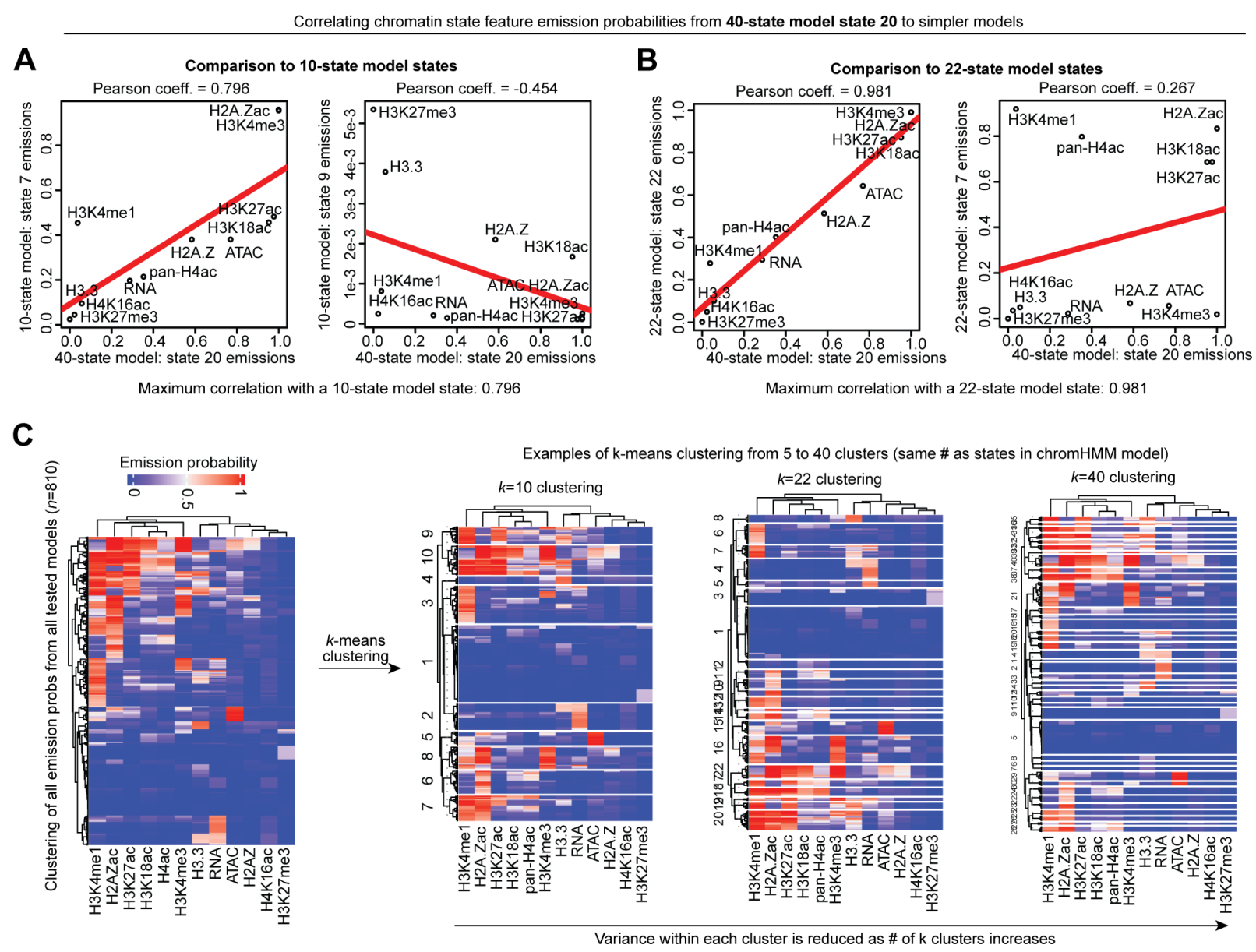

D

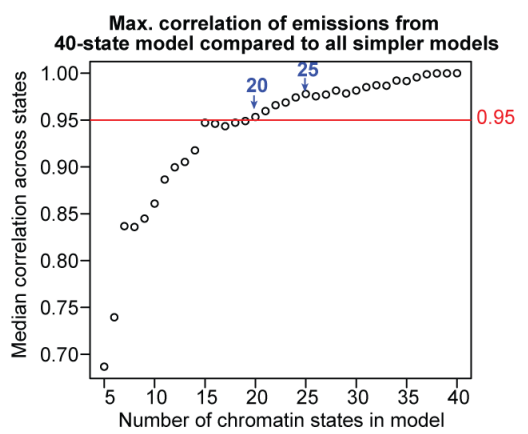

E

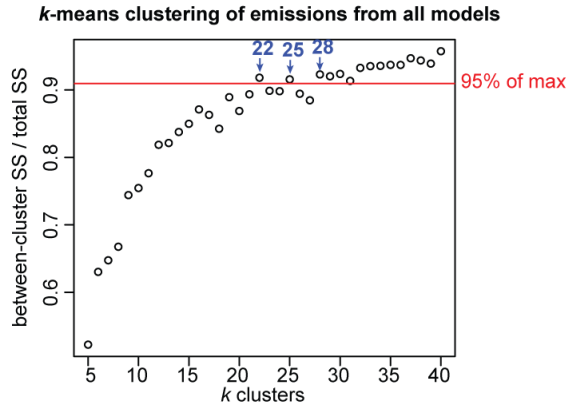

608 Examples and results of quantitative strategies used to select optimal ChromHMM model as

609 devised by Gorkin et al. See Materials and Methods for further details and procedural description.

610 A-B, Correlating chromatin state feature emission probabilities from the most complex model (40-

611 state) to all simpler models. A, Comparison to 10-state model states. The maximum Pearson 
Reske et al.

612 correlation coefficient of 40 -state model state 20 with a 10 -state model state is 0.796 . B,

613 Comparison to 22-state model states. The maximum Pearson correlation coefficient of 40-state

614 model state 20 with a 22-state model state is 0.981 . C, Clustered heatmaps of all emission

615 probabilities from all tested models (left, $n=810$ total modeled chromatin states), followed by

616 examples of $k$-means clustering (right), $k$-means clustering was performed from $k=5$ to $k=40$

617 clusters (the same number of states in the tested chromatin state models) for measurements of

618 goodness of fit by sum-of-squares (between-cluster vs. total). Variance within each cluster is

619 reduced as the number of $k$ clusters increases. D, First strategy model selection plot (as in A-B)

620 based on the maximum correlation of emissions from the most complex model (40-state) compared

621 to all simpler models. The $x$-axis is the simpler model used for comparison, and the $y$-axis is the

622 median of maximum correlation coefficients across all 40-state model states compared to states

623 within the simpler model. 95\% threshold is represented by the red line. E, Second strategy model

624 selection plot (as in $\mathbf{C}$ ) based on the goodness of fit for all simpler models relative to the most

625 complex model (40-state). The $y$-axis is the amount of variance explained by clustering, i.e. the

626 ratio of between-cluster sum-of-squares to total sum-of-squares, relative to $k=40$ clusters (the

627 number of chromatin states in the most complex model). 95\% threshold is represented by the red

628 line. 
Reske et al.

Figure 7-figure supplement 4. Chromatin accessibility repressed by ARID1A is associated with $\mathrm{H} 4$ acetylation.

$\mathbf{A}$

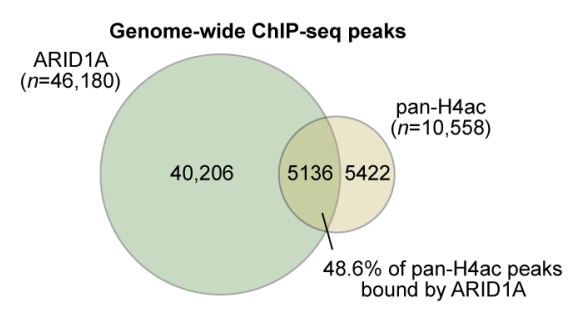

B
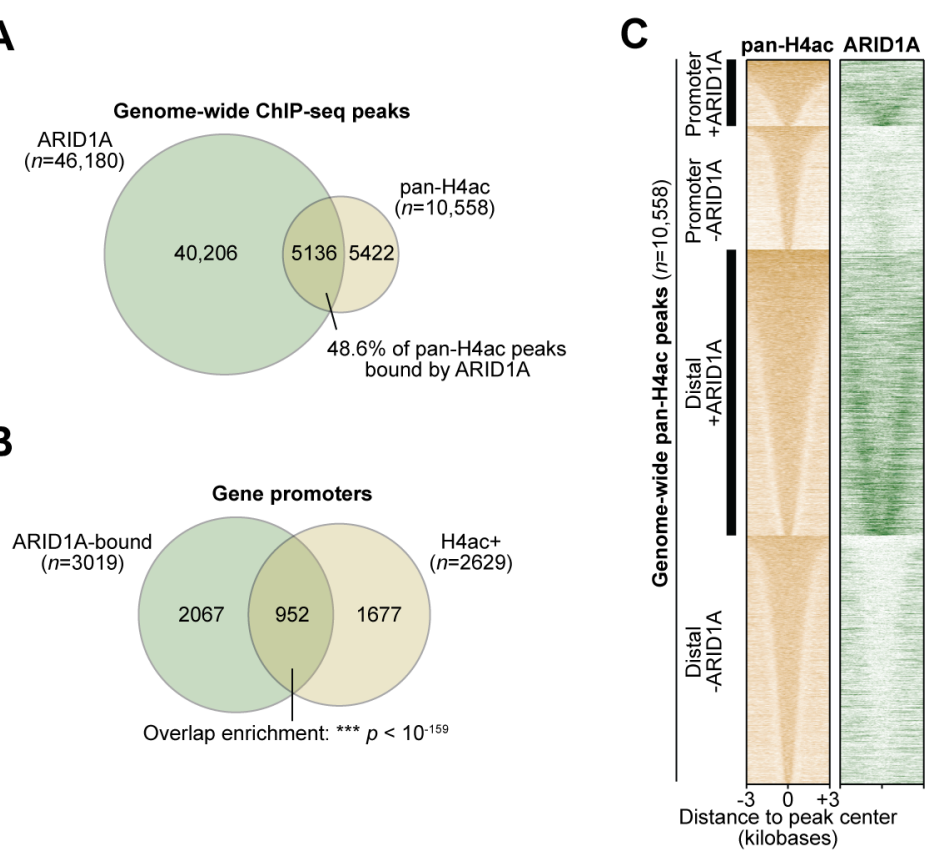

632

E
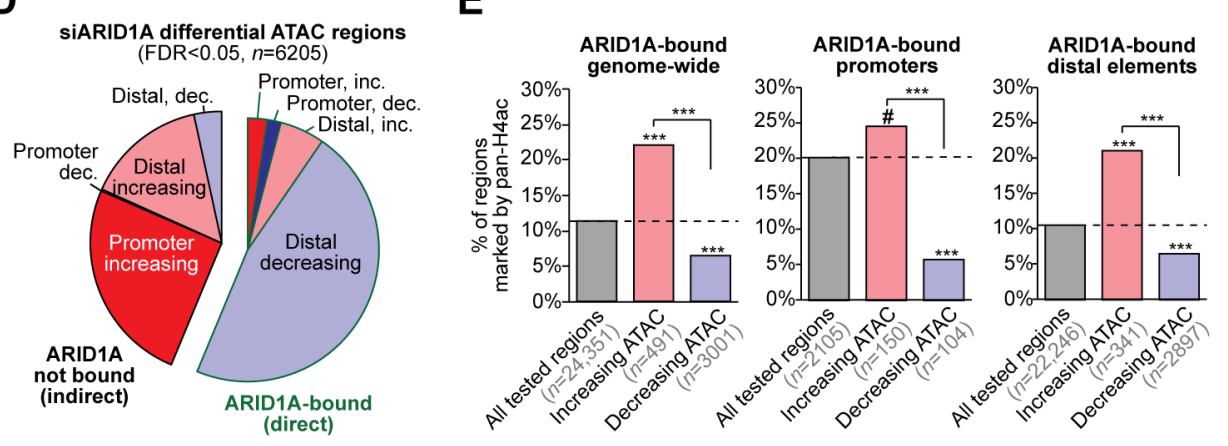

633 A, Euler diagram displaying overlapping genome-wide ChIP-seq peaks (called by MACS2) for

634 pan-H4ac compared to ARID1A. $48.6 \%$ of pan-H4ac peaks are co-marked by ARID1A binding.

B, Euler diagram displaying overlapping gene promoters marked by pan-H4ac $(\mathrm{H} 4 \mathrm{ac}+)$ and

ARID1A binding. Statistic is hypergeometric enrichment test. C, Heatmap displaying ChIP-seq signal across 10,558 genome-wide pan-H4ac ChIP-seq peaks. Signal is quantified as ChIP - Input.

Peaks are ranked by overall pan-H4ac signal and stratified by ARID1A binding and promoter $(<3$ 
Reske et al.

640 significant $($ FDR $<0.05)$ differentially accessible genomic regions, measured by ATAC-seq,

641 following ARID1A depletion via siRNA (siARID1A). Regions are further segregated based on

642 ARID1A binding status. E, Association of pan-H4ac with siARID1A differential ATAC regions

643 directly bound by ARID1A and separated by direction of accessibility change genome-wide (left),

644 at promoters (center), and at distal elements (right). Statistic is hypergeometric enrichment test and

645 two-tailed Fisher's exact test. \# $p<0.10, * * * p<0.001$. 
Reske et al.

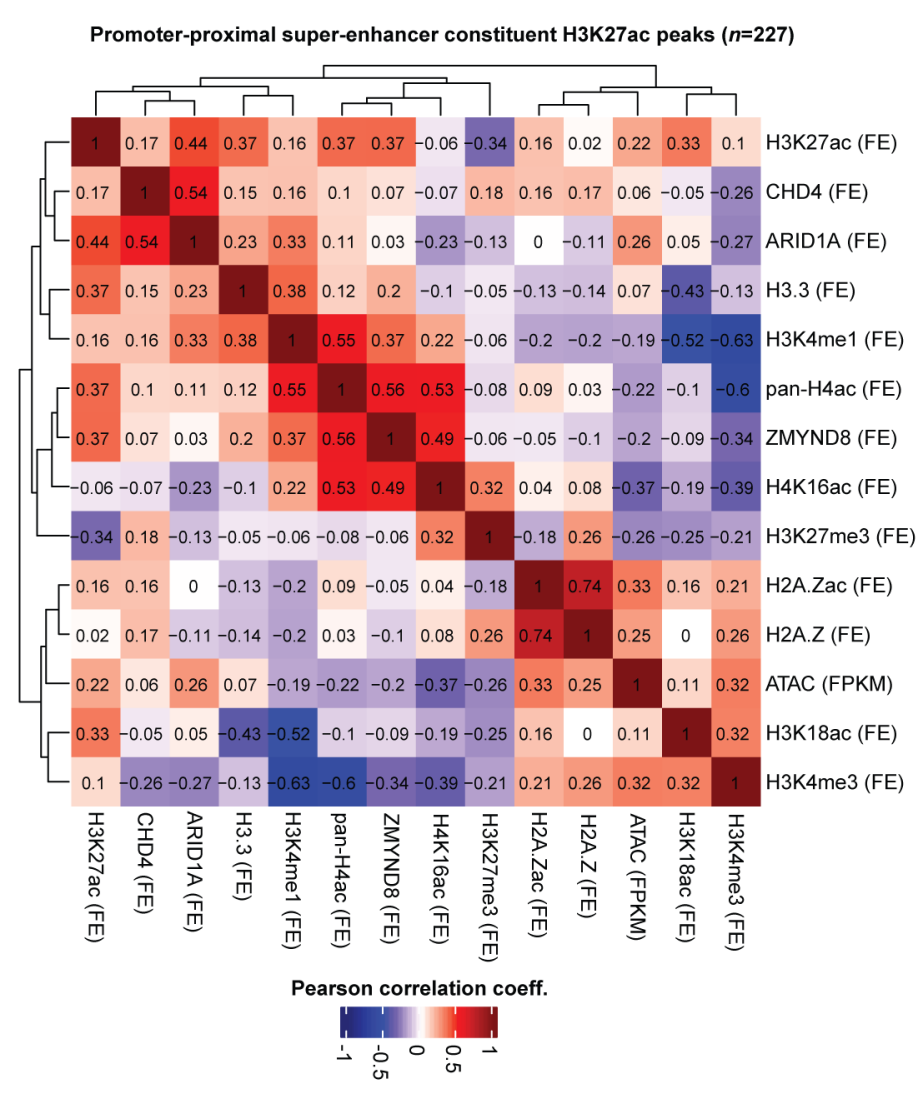

650 Clustered heatmap of Pearson correlation coefficients for all measured chromatin features (aside

651 from total RNA) across 227 promoter-proximal super-enhancer constituent H3K27ac peaks. These

652 active super-enhancer-like regions (distinguished by ROSE) overlap the $3 \mathrm{~kb}$ promoter region

653 flanking a gene TSS. ChIP-seq assays are quantified as ChIP/input chromatin fold-enrichment

654 (FE), and ATAC-seq is quantified as FPKM. 
bioRxiv preprint doi: https://doi.org/10.1101/2022.02.27.482165; this version posted February 28, 2022. The copyright holder for this preprint (which was not certified by peer review) is the author/funder, who has granted bioRxiv a license to display the preprint in perpetuity. It is made available under aCC-BY 4.0 International license.

Reske et al.

Figure 7-figure supplement 6. siCHD4/siZMYND8 functional analysis.

A

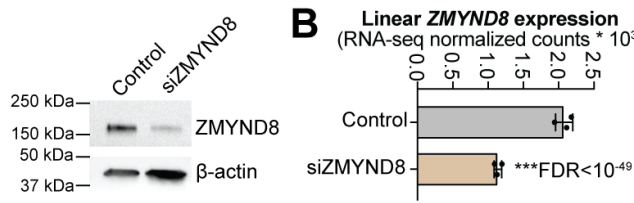

C

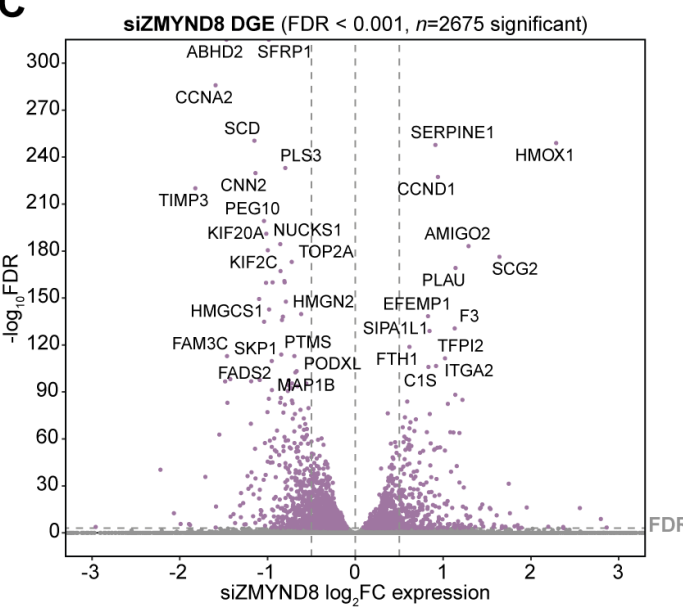

G

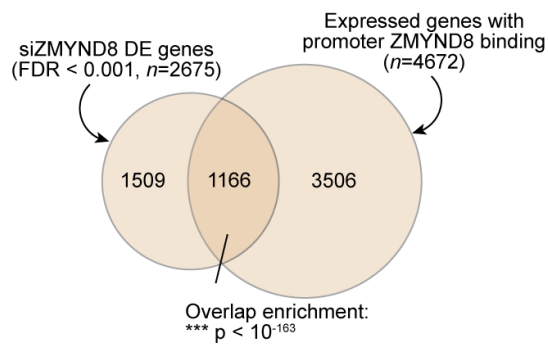

D

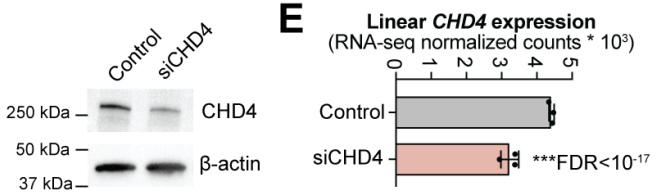

$\mathbf{F}$

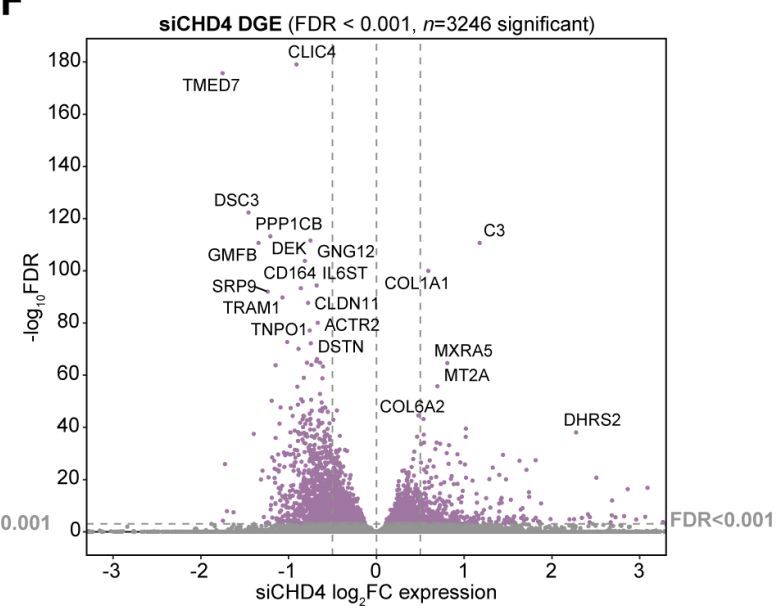

H

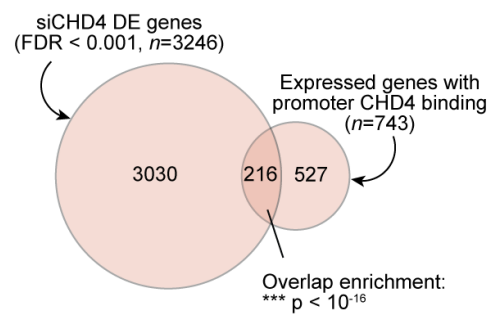

I

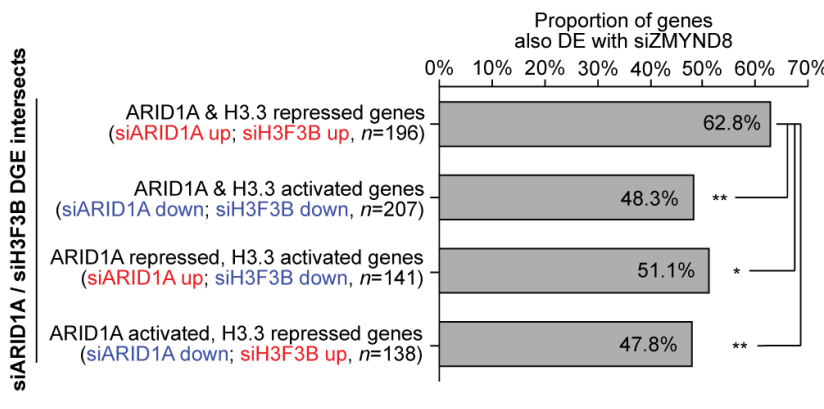

K

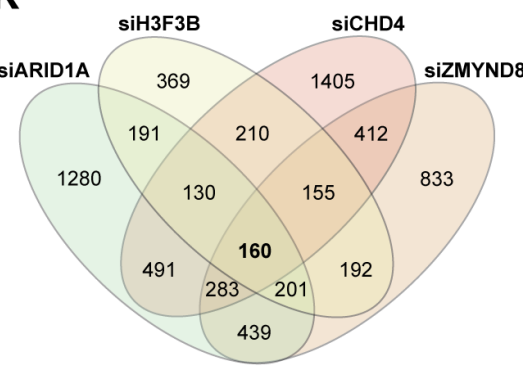

FDR $<0.001$ DGE intersects
Proportion of siZMYND8 DE genes

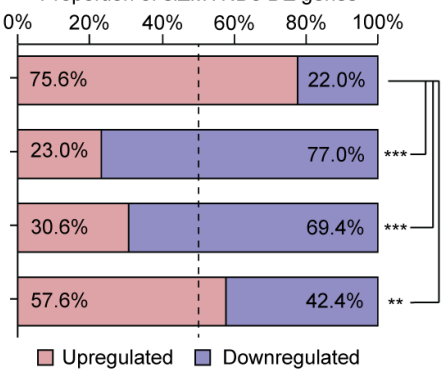

L

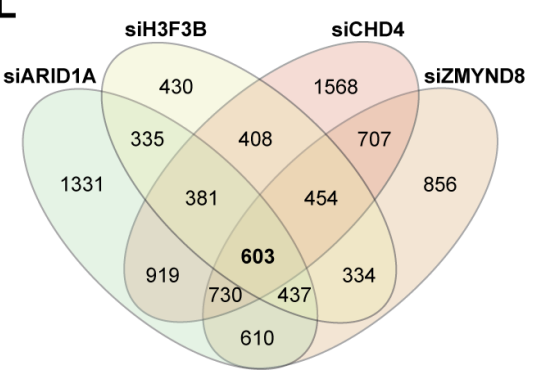

FDR $<0.05$ DGE intersects 
Reske et al.

A, Immunoblot for (top) ZMYND8 compared to (bottom) $\beta$-actin loading control in 12Z cells treated with non-targeting control siRNA or siZMYND8 (ZMYND8 knockdown). B, RNA-seq expression of ZMYND8 in control and siZMYND8 cells. Statistic is FDR-adjusted DESeq2 Wald test. C, Volcano plot for differential gene expression between siZMYND8 and control cells. FDR $<0.001$ was used as a significance threshold. Top significant ZMYND8-dependent genes are labeled. D-F, CHD4 siRNA knockdown (siCHD4) framework and RNA-seq analysis as in A-C.

G, Euler diagram displaying overlap between siZMYND8 differential gene expression (DGE) and ZMYND8 promoter-bound genes. Statistic is hypergeometric enrichment test. H, Euler diagram displaying overlap between siCHD4 DGE and CHD4 promoter-bound genes. Statistic is hypergeometric enrichment test. I, Left, association of siZMYND8 DGE among ARID1A-H3.3 co-regulated gene classes described in Fig. 3F-H. Right, distribution of significantly upregulated (ZMYND8 repressed) vs. downregulated (ZMYND8 activated) siZMYND8 DE genes among DGE (FDR < 0.001) overlap and enrichment across the four analyzed knockdown conditions:

672 siARID1A, siH3F3B, siCHD4, and siZMYND8. The black cell diagonal represents the number of

673 total significant DE genes in that condition. The bottom-left triangle displays the number of

674 overlapping DE genes between pairwise knockdowns. The upper-right triangle displays the

675 overlap enrichment significance by hypergeometric enrichment test. A reduced 18,077 gene set 676 universe was used that contains genes with detected expression in all analyzed conditions. K,

677 Overlap of FDR $<0.001$ DGE sets across the four analyzed knockdown conditions. L, Overlap of

678 FDR $<0.05$ DGE sets across the four analyzed knockdown conditions, corresponding with Fig. $6796 \mathrm{~F}$. 
Reske et al.

Figure 7-figure supplement 7. H4K16ac enrichment at repressed mechanistic genes.

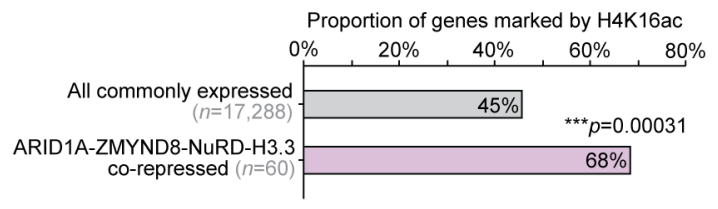

683 Enrichment of H4K16ac at gene promoters or gene bodies of ARID1A-H3.3-ZMYND8-CHD4

684 co-repressed genes - i.e. genes that are upregulated (FDR $<0.05)$ following treatment with

685 siARID1A, siH3F3B, siZMYND8, and siCHD4 — compared to all expressed genes with chromatin 686 data. Statistic is hypergeometric enrichment test. 
Reske et al.

\section{CHD4.}

A
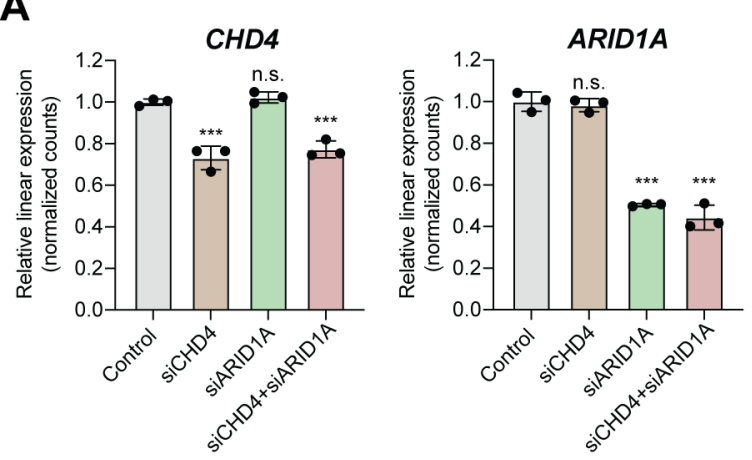

C

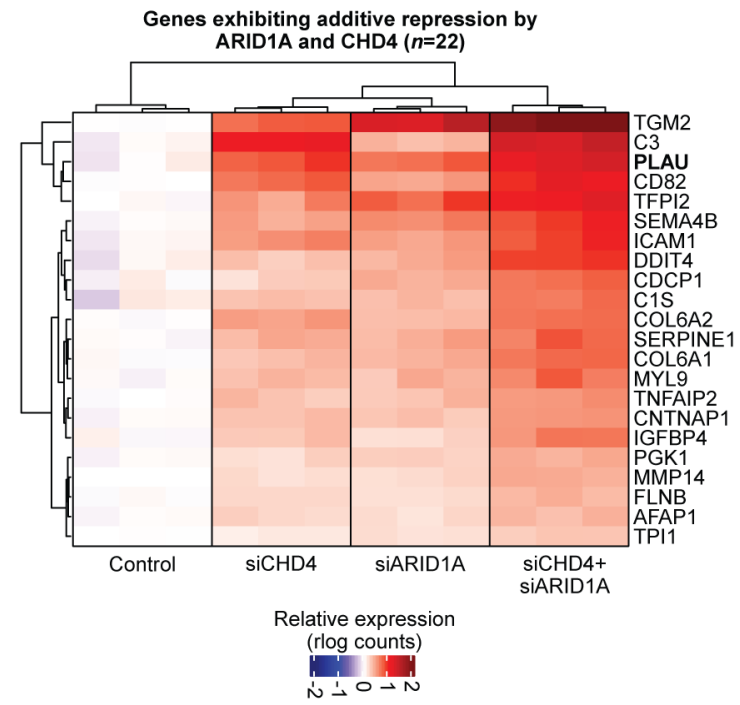

B

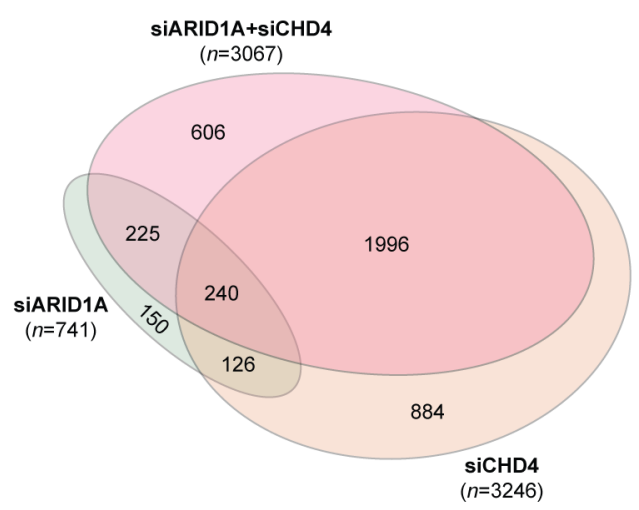

$12 Z$ siRNA vs. control DGE (FDR $<0.001)$

RNA-seq analysis of an ARID1A \pm CHD4 co-knockdown experiment ( $n=3$ per condition). A,

695 cells. C, Clustered heatmap of expression alterations (rlog) at 22 genes displaying additive

696 repression by ARID1A and CHD4. These genes are significantly upregulated in siCHD4, 697 siARID1A, and siARID1A+siCHD4 conditions compared to control cells and 698 siARID1A+siCHD4 vs. single-knockdown conditions. *** FDR $<0.001$ 
bioRxiv preprint doi: https://doi.org/10.1101/2022.02.27.482165; this version posted February 28, 2022. The copyright holder for this preprint (which was not certified by peer review) is the author/funder, who has granted bioRxiv a license to display the preprint in perpetuity. It is made available under aCC-BY 4.0 International license.

Reske et al.

Figure 7-figure supplement 9. Uncropped Western blots from Figure 7-figure

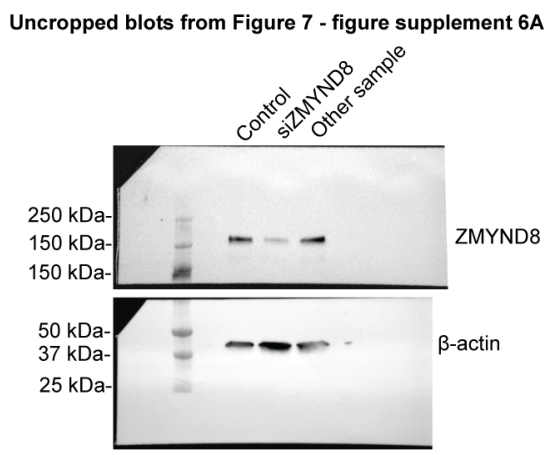

Uncropped blots from Figure 7 - figure supplement 6D

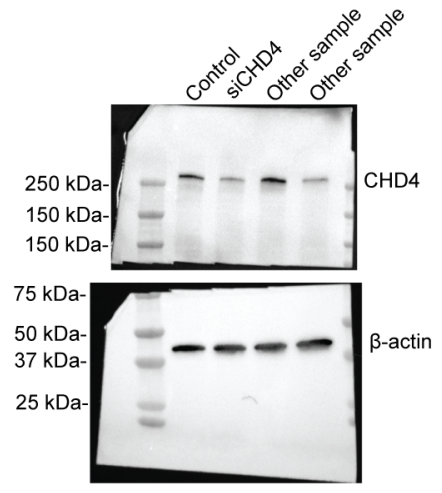


Reske et al.

\section{ARID1A-H3.3 repressed chromatin targets are aberrantly activated in human}

endometriomas.

Our studies in the $12 \mathrm{Z}$ human endometrial epithelial cell line have revealed a mechanism of cooperative chromatin regulation by ARID1A-CHD4-ZMYND8 maintenance of repressive H3.3. To support the relevance of these chromatin regulatory networks on pathologically related gene expression, we utilized a transcriptome expression data set comparing human endometriomas to control endometrial tissue samples (Hawkins et al., 2011). Endometriomas are a result of ectopic spread of endometrial tissue onto the ovary, forming cysts associated with ovarian cancer development (Grandi et al., 2015; Zondervan et al., 2020). Three ARID1A-H3.3 related gene sets were investigated for relevance in human endometrioma gene expression alterations: 1)

713 shARID1A direct decreasing promoter H3.3 genes $(n=412), 2)$ ARID1A-H3.3 co-repressed genes

714 (i.e. siARID1A/siH3F3B upregulated, FDR < 0.001, $n=196$ ), and 3) ARID1A-H3.3-CHD4-

715 ZMYND8 co-repressed genes (i.e. upregulated with any knockdown, FDR $<0.05, n=60$ ). We observed significant enrichment for all three of these gene sets among human endometrioma DGE

717 (Figure 8A, left). Moreover, the overlapping DE genes were more likely to be upregulated in 718 endometriomas than expected by chance, indicating relief of repression is also observed in 719 pathology (Figure 8A, right). Similarly, examining the endometrioma vs. control endometrium 720 expression $\log _{2} \mathrm{FC}$ values indicated that each gene set tended to be overall upregulated in the 721 pathological, pre-cancerous state (Figure 8B). Mechanistic genes aberrantly activated in

722 endometriomas that could be attributed to disruption of ARID1A-H3.3 chromatin repression 723 mechanisms include $C 1 S, S C A R B 1, G Y P C, W W C 3, C O L 6 A 2$, and $M A P 4 K 4$ (Figure 8C). Our data 724 indicate that ARID1A cooperatively maintains transcriptionally repressive $\mathrm{H} 3.3$ chromatin 725 through protein-protein interactions with CHD4 and ZMYND8, likely recruited to H4(K16)ac+ 
bioRxiv preprint doi: https://doi.org/10.1101/2022.02.27.482165; this version posted February 28, 2022. The copyright holder for this preprint (which was not certified by peer review) is the author/funder, who has granted bioRxiv a license to display the preprint in perpetuity. It is made available under aCC-BY 4.0 International license.

Reske et al.

726 chromatin, such that loss of any of these factors leads to alleviation of H3.3-mediated repression

727 and consequential aberrant gene activation in various endometrial disease contexts where ARID1A

728 mutations are thought to drive pathogenesis.

729 
Reske et al.

Figure 8. Mechanistic gene expression alterations in human endometriomas.

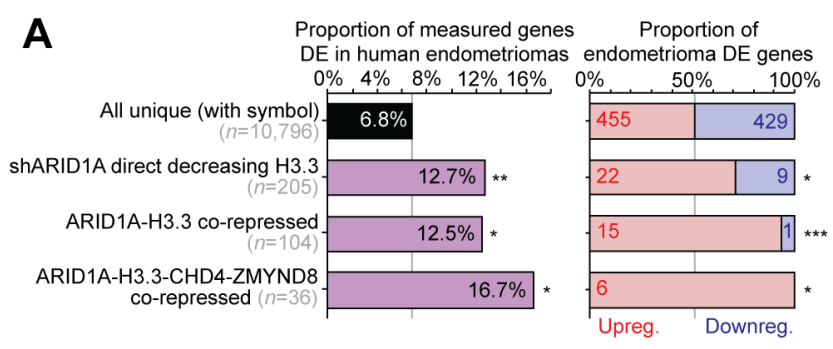

B

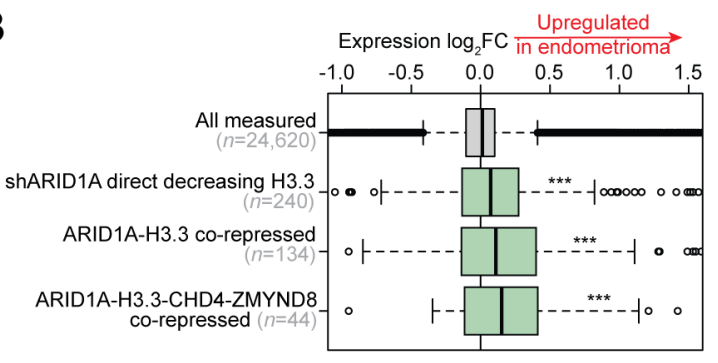

C

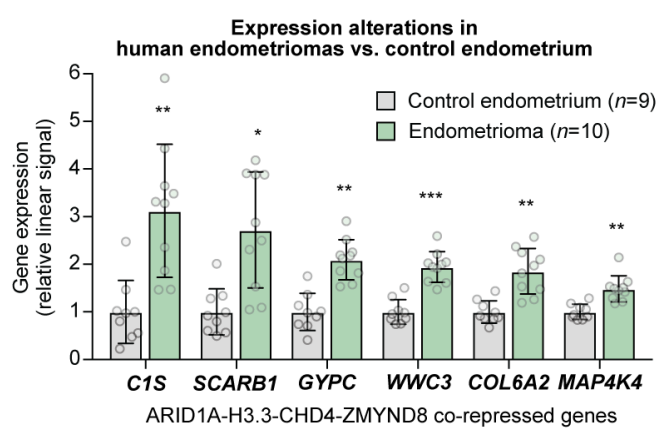

732 A, Left, enrichment for ARID1A-H3.3 co-repressive chromatin mechanistic gene sets among

733 human endometrioma (ovarian endometriosis) vs. control endometrium DE genes reported by

734 Hawkins et al. (Hawkins et al., 2011), compared to all unique measured genes. Right, proportion

735 of overlapping DE genes that are upregulated vs. downregulated in endometriomas, compared to

736 all unique measured genes. Statistic is hypergeometric enrichment. B, Box plots displaying

737 endometrioma expression $\log _{2} \mathrm{FC}$ values for probes annotated to genes within mechanistic gene

738 sets, compared to all measured probes. Statistic is two-tailed, unpaired Wilcoxon's test. C, Relative

739 expression box-dot plots of 6 genes upregulated in endometriomas vs. control endometrium that

740 are co-repressed by ARID1A, H3.3, CHD4, and ZMYND8. Statistic is limma FDR-adjusted $p$. *

$741 p<0.05, * * p<0.01, * * * p<0.001$. 
Reske et al.

\section{Discussion}

We have provided evidence that ARID1A functions to maintain the variant histone H3.3

744 in active chromatin. ARID1A loss leads to H3.3 depletion at active enhancers and super-enhancers,

745 due to disrupted ARID1A chromatin interactions, leading to H3.3 redistribution toward active

746 genic and transcribed elements. SWI/SNF functions to eject nucleosomes and open chromatin,

747 rather than assemble nucleosomes on chromatin. SWI/SNF disruption of nucleosomes may be

748 required for H3.3 incorporation and thus be coupled to nucleosome assembly. Therefore, we

749 hypothesize that H3.3 regulation by ARID1A-SWI/SNF occurs by ejecting canonical H3.1

750 containing nucleosomes in favor of H3.3 incorporation by other assembly or chaperone factors. In

751 the absence of ARID1A-SWI/SNF, H3.3 nucleosome assembly may be impeded. Given previous

752 associations between H3.3 epigenetic memory and cell fate plasticity, it is intriguing to consider a

753 role for ARID1A-SWI/SNF regulation of H3.3 as being a critical determinant of endometrial

754 epithelial cell identity and homeostasis across the menstrual cycle when proliferation and 755 differentiation occur (Szenker et al., 2011).

ARID1A maintenance of $\mathrm{H} 3.3$ is associated genomic interactions with CHD4, a catalytic

757 subunit in the SWI/SNF-like NuRD remodeler complex. Target regulatory activity is further

758 directed by CHD4 interactions with histone reader ZMYND8, where ZMYND8 is recruited to

759 H4(K16)ac-marked chromatin, possibly through its bromodomain. This ZMYND8-mediated

760 targeting of ARID1A-CHD4 to H3.3 is associated with repressive chromatin regulation, notably

761 at super-enhancers located upstream of genes, such that disruption of this chromatin mechanism

762 causes relief of repression and subsequent transcriptional hyperactivation. Plasminogen activator

763 urokinase $(P L A U)$ was identified as a key target gene repressed by this mechanism in our $12 \mathrm{Z}$

764 endometriotic epithelial cell model. PLAU was also recently observed as transcriptionally 
Reske et al.

765 activated during human menstruation (Wang et al., 2020), suggesting similar repressive chromatin

766 mechanisms may govern PLAU regulation in the healthy endometrium. PLAU is upregulated in

767 ovarian endometrioid carcinomas from women with concurrent endometriosis (C. Zhang et al.,

768 2018), suggesting PLAU upregulation may promote malignant transformation in endometriosis.

769 ARID1A mutations are frequently observed in endometriosis-associated ovarian cancers (Jones et

770 al., 2010; Wiegand et al., 2010). C1S, a component of the complement C1 complex, is another

771 gene that is transcriptionally repressed by ARID1A, CHD4, ZMYND8, and H3.3 that is aberrantly

772 upregulated in human endometriomas. It has been reported that the complement system is activated

773 in women with endometriosis (Sikora et al., 2018), suggesting that ARID1A mutation and

774 associated disruption of chromatin repression may be a possible disease mechanism.

775 H3.3 is considered an active chromatin mark associated with transcriptional activation.

776 However, our data and others have demonstrated that H3.3 can play roles in transcriptional

777 repression, as well as transcriptional poising and higher-order chromatin regulation, although the

778 mechanisms governing these functional specificities remain unclear (Shi et al., 2017). A simple

779 hypothesized mechanism explaining how H3.3 can function repressively is through associations

780 with CHD4 and the NuRD complex, as we have studied here. Historically, NuRD has been studied

781 as a repressor due to its subunit composition that includes the histone deacetylases HDAC1/2,

782 although activating roles of NuRD are also known (Basta \& Rauchman, 2015; Tong, Hassig,

783 Schnitzler, Kingston, \& Schreiber, 1998; Xue et al., 1998). An early study of H3.3 chromatin

784 dynamics indicated that NuRD components were associated with active regions marked by high

785 H3.3 turnover (Ha, Kraushaar, \& Zhao, 2014). More recently, NuRD has been shown to directly

786 interact with H3.3 nucleosomes (Kraushaar et al., 2018). Further, we have shown that ARID1A 
Reske et al.

787

788

789

790

791

792

793

794

795

796

797

798

799

800

801

802

803

804

805

806

807

808

809

physically interacts with CHD4 in endometrial epithelial cells, and ARID1A and CHD4 co-binding at unique chromatin sites is associated with H3.3 maintenance and transcriptional repression.

Despite these observations, it is not clear how ARID1A cooperates with CHD4 through protein-protein interactions to regulate $\mathrm{H} 3.3$ nucleosomes. Intriguingly, $\mathrm{CHD} 4 / \mathrm{NuRD}$ was recently shown to control super-enhancer accessibility and maintain lower acetylation levels through its HDAC activity (Marques et al., 2020), similar to our findings with ARID1A by antagonizing P300 (Wilson et al., 2020). In support of our data, the authors observed CHD4 interactions with SWI/SNF. Recently, NuRD and SWI/SNF recruitment to active TSS and enhancers was impaired in H3.3K4A mutant mouse ESCs (Gehre et al., 2020), suggesting that NuRD and SWI/SNF recruitment is dependent on the K4 residues on H3.3. The same study also reported the K4A mutation destabilized H3.3 and that both CHD4 and SMARCA4 (SWI/SNF) are required for H3.3 stability, further supporting a role for NuRD and SWI/SNF in H3.3 maintenance (Gehre et al., 2020). Loss of H3.3 following disruption of ARID1A chromatin interactions could lead to destabilization of repressive NuRD. One hypothesized cooperative mechanism that could explain how ARID1A and CHD4 maintain H3.3 in active chromatin is through different aspects of remodeler activity conferred by each complex. ARID1A could remodel or remove canonical H3 nucleosomes - a typical role of SWI/SNF - while CHD4 could function to assemble or stabilize H3.3. In this scenario, failure to remove canonical H3 following ARID1A loss may impair the ability of CHD4 to assemble or stabilize H3.3, and thus loss of either ARID1A or CHD4 may break this cycle. The related CHD1 remodeler is known to be required for H3.3 deposition into chromatin in vivo (Konev et al., 2007), further suggesting a necessary role for remodeler activity in H3.3 nucleosome assembly. It is important to note that H3.3 has a defined chaperone associated with transcriptional activity, HIRA (Shi et al., 2017). P400 is another SWI/SNF-like remodeler 
Reske et al.

recently shown to exchange H3.3 nucleosomes that could also possibly collaborate with SWI/SNF

811 (Pradhan et al., 2016).

In silico analyses from the ReMap 2020 transcriptional regulator peak database (Cheneby

813 et al., 2020) predicted that ZMYND8 is highly associated with H3.3 chromatin regulation by

814 ARID1A. While we did not detect physical interactions directly between ARID1A and ZMYND8

815 under high salt conditions, we did observe ARID1A-CHD4 and CHD4-ZMYND8 interactions.

816 ZMYND8 has been shown to interact with NuRD in numerous contexts (Adhikary et al., 2016;

817 Gong et al., 2015; Savitsky et al., 2016; Spruijt et al., 2016). Intriguingly, one recent study reported that ZMYND8 directly recognizes mutant H3.3G34R (Jiao et al., 2020). Our data indicate that ZMYND8 links repressive H3.3 to H4 acetylation. In support, the ZMYND8 bromodomain

820 directly interacts with acetylated H4 tails (Adhikary et al., 2016), and TIP60-mediated H4

821 acetylation can functionally recruit ZMYND8 through this mechanism to repress transcription with

822 CHD4 in response to DNA damage (Gong et al., 2015). H4K16ac was also previously reported to

823 be required for the NoRC repressor complex to bind and silence chromatin (Zhou \& Grummt,

824 2005). Our data also indicate that ARID1A directly suppresses chromatin accessibility at sites

825 marked by $\mathrm{H} 4$ acetylation, suggesting that SWI/SNF chromatin remodeler activity may be

826 involved in ZMYND8-NuRD-mediated chromatin repression. Interestingly, in addition to NuRD-

827 dependent regulation, ZMYND8 regulates transcription independently of NuRD (Adhikary et al.,

828 2016). Positive transcriptional regulation by ZMYND8 has been shown to occur through its

829 association with the P-TEFb elongation complex (Ghosh et al., 2018). ZMYND8-NuRD

830 repression in response to DNA damage was previously shown to rely on KDM5A demethylase

831 activity (Gong, Clouaire, Aguirrebengoa, Legube, \& Miller, 2017), further suggesting other factors

832 may orchestrate this regulatory activity. ZMYND8 has been reported to be a super-enhancer factor 
Reske et al.

833 that suppresses hyperactivation (Shen et al., 2016). Corroborating our results, ZMYND8 was

834 previously shown to associate with NuRD at super-enhancers (Spruijt et al., 2016). We found that

835 super-enhancers that become hyperacetylated following ARID1A loss are normally associated

836 with the highest levels of H3.3 and ZMYND8 binding. In our proposed model, ZMYND8

837 bromodomain interactions with $\mathrm{H} 4$ acetylated tails recruit CHD4 and ARID1A for transcriptional

838 repression at active chromatin. This most notably occurs at H3.3+ super-enhancers, where all three

839 factors co-localize most frequently. Further work will seek to explain how ZMYND8 specifies this

840 repressive activity, as CHD4 and ARID1A can also function toward transcriptional activation.

841

It is worth considering alternative hypotheses of how transcriptional homeostasis could be

842 altered following disruption of these chromatin regulators and epigenomic marks. In addition to

843 promoter and enhancer chromatin regulation, SWI/SNF, NuRD, and ZMYND8 have been shown

844 to mediate transcriptional pausing and elongation by Pol II and associated machinery (Bottardi et

845 al., 2014; Ghosh et al., 2018; Schwabish \& Struhl, 2007; Trizzino et al., 2018), as well as DNA

846 repair (Gong et al., 2015; Park et al., 2006; Smeenk et al., 2010). Super-enhancers mark critical

847 cell identity genes (Whyte et al., 2013), and recent evidence suggests chromatin mechanisms

848 coupling transcription and DNA repair occur at super-enhancers to control transcriptional

849 hyperactivation (Hazan, Monin, Bouwman, Crosetto, \& Aqeilan, 2019). Super-enhancer

850 chromatin co-regulation by ARID1A, CHD4, and ZMYND8 may fine tune transcriptional

851 activation states and thus reflect a mechanism at the intersection of transcriptional regulation and

852 DNA repair. 
Reske et al.

\section{Materials and Methods}

\section{Data availability}

856 All new data generated in this study have been deposited to the Gene Expression Omnibus (GEO)

857 at SuperSeries accession GSE190557, and access is available with reviewer token:

858 ijmtkuuivtwzbwz. Previously generated and re-analyzed data were retrieved from GEO at 859 accessions GSE121198 and GSE148474 and analyzed as previously described (Wilson et al., 860 2020; Wilson et al., 2019).

\section{Cell culture, siRNA transfections, and lentiviral shRNA particle usage.}

863 Adherent, human $12 \mathrm{Z}$ endometriotic epithelial cells were cultured in DMEM/F12 media in the 864 presence of $10 \%$ serum (FBS), 1\% L-glutamine, and 1\% penicillin/streptomycin. Cells were 865 seeded in antibiotic-free media the day before siRNA transfection. $50 \mathrm{nM}$ siRNA (Dharmacon, 866 ON-TARGETplus) were transfected into cells using the Lipofectamine RNAiMAX 867 (ThermoFisher Scientific) reagent, according to the manufacturer protocol, in OptiMEM (Gibco).

868 Growth media was replaced 24 hours following transfection, without antibiotics. 48 hours after 869 transfection, low serum $(0.5 \%$ FBS $)$ growth media was added with antibiotics. Cells were 870 harvested 72 hours following siRNA transfection. Lentiviral shRNA particles were prepared with

871 Lenti-X 293T cells (Takara) and MISSION pLKO.1 plasmids (Sigma-Aldrich) as previously 872 described (Wilson et al., 2020). Lentiviral shRNA particles were titered using the qPCR Lentiviral 873 Titration Kit (ABM). shRNA particles were transduced into $12 \mathrm{Z}$ cells at a 100-fold multiplicity of 874 infection, and media was replaced 24 hours later. Cells were harvested 72 hours following shRNA 875 transduction. 
Reske et al.

\section{Cell cycle analysis.}

878 The Click-iT Plus EdU Flow cytometry Assay Kit (Invitrogen) was used for cell cycle assays. 12Z

879 cells were treated with $10 \mathrm{mM}$ of EdU for 2 hours in culture media. Cells were harvested by 880 trypsinization and washed in 1\% BSA in PBS. Cells were resuspended in $100 \mu \mathrm{L}$ of ice-cold PBS, 881 and $900 \mu \mathrm{L}$ of ice cold $70 \%$ ethanol was added dropwise while vortexing. Cells were incubated on 882 ice for two hours. Cells were washed with 1\% BSA in PBS and then treated with the Click-iT Plus 883 reaction cocktail including Alexa Fluor 488 picolyl azide according to the manufacturer's 884 instructions for 30 minutes. Cells were washed with 1X Click-iT permeabilization buffer and wash 885 reagent, and then treated with $5 \mathrm{mM}$ of Vybrant DyeCycle Ruby Stain (ThermoFisher) diluted in $1 \%$ BSA in PBS for 30 minutes at $37^{\circ} \mathrm{C}$. Flow cytometry was performed using a BD Accuri C6

887 flow cytometer (BD Biosciences) and analyzed using FlowJo v10 software (BD Biosciences).

\section{Histone extraction.}

$12 \mathrm{Z}$ cells were washed with PBS and scraped in PBS containing $5 \mathrm{mM}$ sodium butyrate. Cells

891 were centrifuged and resuspended in TEB buffer (PBS supplemented with 0.5\% Triton X-100, 5

$892 \mathrm{mM}$ sodium butyrate, $2 \mathrm{mM}$ phenylmethylsulfonyl fluoride, $1 \mathrm{X}$ protease inhibitor cocktail) and

893 incubated on a 3D spindle nutator at $4{ }^{\circ} \mathrm{C}$ for 10 minutes. Cells were centrifuged at $3000 \mathrm{RPM}$ for

89410 minutes at $4{ }^{\circ} \mathrm{C}$. TEB wash step was repeated once. Following second wash, pellet was 895 resuspended in $0.2 \mathrm{~N} \mathrm{HCl}$, and incubated on $3 \mathrm{D}$ spindle nutator at $4{ }^{\circ} \mathrm{C}$ overnight. The following 896 day, samples were neutralized with 1:10 volume 1M Tris-HCl pH 8.3. Sample was centrifuged at 8973000 RPM for 10 minutes at $4{ }^{\circ} \mathrm{C}$, and supernatant containing histone proteins was collected. 
Reske et al.

\section{Co-immunoprecipitation (co-IP).}

901 Nuclear extracts were prepared as previously described (Chandler et al., 2013), dialyzed overnight into 0\% glycerol (25 mM HEPES, 0.1 mM EDTA, $12.5 \mathrm{mM} \mathrm{MgCl2,} 100 \mathrm{mM} \mathrm{KCl,} 1 \mathrm{mM}$ DTT)

903 using a Slide-A-Lyzer G2 Dialysis Cassette (10 kDa cutoff, ThermoFisher Scientific), and 904 quantified with the BCA Protein Assay Kit (Pierce, ThermoFisher Scientific). Primary antibodies 905 (anti-ARID1A, D2A8U, Cell Signaling; anti-CHD4, D8B12, Cell Signaling;) were conjugated to 906 Protein A Dynabeads (Invitrogen) overnight at $4{ }^{\circ} \mathrm{C}$ in $1 \mathrm{X}$ PBS $+0.5 \% \mathrm{BSA}$. Normal rabbit IgG 907 (Cell Signaling) IPs were performed in parallel at equivalent masses, as negative controls. $500 \mu \mathrm{g}$ 908 nuclear lyase was diluted into IP buffer (20 mM HEPES, $150 \mathrm{mM} \mathrm{KCl,} \mathrm{10 \%} \mathrm{glycerol,} 0.2 \mathrm{mM}$ EDTA, $0.1 \%$ Tween-20, $0.5 \mathrm{mM}$ DTT) to a final volume of $1 \mathrm{~mL}$ and clarified by centrifugation.

910 After overnight IP at $4{ }^{\circ} \mathrm{C}$, bead slurries were washed with a series of IP buffers at different $\mathrm{KCl}$ 911 concentrations: $2 \mathrm{X}$ washes at $150 \mathrm{mM}, 3 \mathrm{X}$ washes at $300 \mathrm{mM}, 2 \mathrm{X}$ washes at $100 \mathrm{mM}, 1 \mathrm{X}$ wash

912 at $60 \mathrm{mM}$. Immunoprecipitants were eluted in $2 \mathrm{X}$ Laemmli buffer $+100 \mathrm{mM}$ DTT at $70{ }^{\circ} \mathrm{C}$ for 10 913 minutes with agitation.

\section{Glycerol gradient sedimentation.}

916 Nuclear extracts were prepared, dialyzed, and quantified as described in the co-IP methods section.

917 Density sedimentation by glycerol gradient was performed and probed similar to published reports 918 (Mashtalir et al., 2018). Briefly, $4.5 \mathrm{~mL}$ 10-30\% linear glycerol gradients were prepared using an 919 ÄKTA start (Cytiva) from density sedimentation buffer (25 mM HEPES, 0.1 mM EDTA, 12.5 $920 \mathrm{mM} \mathrm{MgCl} 2,100 \mathrm{mM} \mathrm{KCl}, 1 \mathrm{mM} \mathrm{DTT}$ ) additionally containing 30\% and 10\% glycerol for initial 921 and target concentrations, respectively. $200 \mu \mathrm{g}$ nuclear lyase was overlaid on the glycerol gradient 922 followed by ultracentrifugation at 40,000 rpm in an AH-650 swinging bucket rotor (ThermoFisher 
Reske et al.

923 Scientific) for 16 hours at $4{ }^{\circ} \mathrm{C} .225 \mu \mathrm{L}$ gradient fractions were collected and concentrated using

924 StrataClean resin (Agilent). Concentrated fractions were eluted in 1.5X Laemmli buffer +37.5

925 mM DTT and run on SDS-PAGE for immunoblotting.

926

927 Immunoblotting.

928 Whole-cell protein lysates were prepared as previously described (Wilson et al., 2020). Proteins 929 were quantified with the BCA Protein Assay Kit (Pierce, ThermoFisher Scientific). Protein 930 samples in Laemmli buffer + DTT were denatured at $94{ }^{\circ} \mathrm{C}$ for 3 minutes prior to running on SDS-

931 PAGE gels (6\% gels for co-IP and glycerol gradients, 15\% gels for histone extracts, and 4-15\%

932 gradient gels for whole-cell protein lysates). Gels containing histone extracts were wet transferred

933 to nitrocellulose membranes at $4{ }^{\circ} \mathrm{C}$ for 3 hours at $400 \mathrm{~mA}$ current, then dried at room temperature

934 followed by re-hydration in TBS $+0.1 \%$ Tween-20 (TBS-T) and blocking with Odyssey blocking

935 buffer (LI-COR). All other gels were semi-dry transferred to PVDF using a Trans-Blot Turbo (Bio-

$936 \mathrm{Rad}$ ) according to the manufacturer's protocol designed for high-molecular weight proteins, and

937 blocked with either $5 \%$ BSA or $5 \%$ milk in TBS. The following primary antibodies were used:

938 anti-ARID1A (D2A8U, Cell Signaling), anti-CHD4 (D4B7, Cell Signaling), anti-ZMYND8

939 (A302-089, Bethyl), anti-ZMYND8 (Atlas), anti-BRG1 (ab110641, abcam), anti-BAF155

940 (D7F8S, Cell Signaling), anti-HDAC1 (10E2, Cell Signaling), anti-histone H3.3 (ab176840,

941 abcam), anti-histone H3.3 (2D7-H1, abnova), and anti-histone H3 (D1H2, Cell Signaling). IRDye

942 fluorescent dye (LI-COR) secondary antibodies were used for LI-COR fluorescence-based protein

943 visualization of histones. Horseradish peroxidase (HRP) conjugated secondary antibodies (Cell

944 Signaling) were used for chemiluminescence-based protein visualization of all other targets. 
Reske et al.

945 Clarity Western ECL substrate (Bio-Rad) was used to activate HRP for chemiluminescence, captured by ChemiDoc XRS+ imaging system (Bio-Rad).

\section{mRNA-seq and analysis.}

72 hours after initial siRNA transfection, and 24 hours after low-sera conditioning, 12Z cells were purified for RNA using the Quick-RNA Miniprep Kit (Zymo Research). Transcriptome libraries

951 ( $n=3$ replicates) were prepared and sequenced by the Van Andel Genomics Core from $500 \mathrm{ng}$ of 952 total RNA using the KAPA mRNA HyperPrep kit (v4.17) (Kapa Biosystems). RNA was sheared to $300-400 \mathrm{bp}$. Prior to PCR amplification, cDNA fragments were ligated to IDT for Illumina unique dual adapters (IDT DNA Inc). Quality and quantity of the finished libraries were assessed 955 using a combination of Agilent DNA High Sensitivity chip (Agilent Technologies), QuantiFluor dsDNA System (Promega), and Kapa Illumina Library Quantification qPCR assays (Kapa

957 Biosystems). Individually indexed libraries were pooled and $50 \mathrm{bp}$, paired-end sequencing was 958 performed on an Illumina NovaSeq 6000 sequencer using a 100-cycle sequencing kit (Illumina).

959 Each library was sequenced to an average raw depth of 20-25 million reads. Base calling was done 960 by Illumina RTA3 and output of NCS was demultiplexed and converted to FastQ format with

961 Illumina Bcl2fastq v1.9.0.

962 For analysis, briefly, raw reads were trimmed with cutadapt (Martin, 2011) and Trim

963 Galore! (http://www.bioinformatics.babraham.ac.uk/projects/trim_galore/) followed by quality

964 control analysis via FastQC (Andrews, 2010) and MultiQC (Ewels, Magnusson, Lundin, \& Kaller, 965 2016). Trimmed reads were aligned to hg38 assembly and indexed to GENCODE (v28) along with 966 gene feature counting via STAR (Dobin et al., 2013). Low count genes with less than 1 count per 967 sample on average were filtered prior to count normalization and differential gene expression 
Reske et al.

968 (DGE) analysis by DESeq2 with empirical Bayes shrinkage for fold-change estimation (Love,

969 Anders, Kim, \& Huber, 2015; Love, Huber, \& Anders, 2014). Wald probabilities were corrected

970 for multiple testing by independent hypothesis weighting (IHW) (Ignatiadis, Klaus, Zaugg, \&

971 Huber, 2016) for downstream analyses. In presented analyses, " $\log _{2} \mathrm{FC} "$ is the empirically

972 observed $\log _{2}$ fold-change in expression between conditions, while "s $\log _{2} \mathrm{FC} "$ is a moderated $\log _{2}$

973 fold-change estimate that removes noise from low count genes using the apeglm shrinkage

974 estimator as implemented in DESeq2 (Zhu, Ibrahim, \& Love, 2019). Pairwise comparisons

975 between different DGE analyses and gene sets were initially filtered for genes with transcripts

976 commonly detected in both cell populations.

978 Histone peptide arrays.

979 Anti-acetyl-H2A.Z (K4/K7) (D3V1I, Cell Signaling) antibody specificity was analyzed via histone 980 peptide microarrays as previously described (Cornett, Dickson, \& Rothbart, 2017) with minor 981 modifications. Arrays were designed in ArrayNinja (Dickson, Cornett, Ramjan, \& Rothbart, 2016)

982 and printed using a 2470 Arrayer (Quanterix). All hybridization and wash steps were performed 983 at ambient temperature. Slides were blocked with hybridization buffer (1X PBS [pH 7.6], 0.1\%

984 Tween, 5\% BSA) for 30 minutes, then incubated with primary antibody diluted 1:1000 in 985 hybridization buffer for 1 hour. Slides were washed $3 X$ for 5 minutes with PBS, then probed with 986 Alexa647-conjugated secondary antibody diluted 1:5000 in hybridization buffer for 30 minutes.

987 Slides were washed $3 X$ for 5 minutes with PBS, dipped in 0.1X PBS to remove salt, and spun dry.

988 Slides were scanned on an InnoScan 1100 microarray scanner (Innopsys) and images were 989 analyzed and quantified using ArrayNinja. Plots were generated in Prism (GraphPad). Each 
Reske et al.

peptide antigen is printed six times per array, and each antibody was screened on two separate arrays.

\section{Chromatin immunoprecipitation (ChIP-seq) and analysis.}

994 Wild-type and lentiviral shRNA particle transduced $12 Z$ cells were treated with $1 \%$ formaldehyde

995 in growth media for 10 minutes at ambient temperature. Formaldehyde was quenched by the addition of $0.125 \mathrm{M}$ Glycine and incubation for 5 minutes at room temperature, followed by PBS wash and scraping. 1*107 crosslinked cells were used for each ChIP, and each antibody and condition for ChIP was performed in duplicate. Chromatin from crosslinked cells was fractionated by digestion with micrococcal nuclease using the SimpleChIP Enzymatic Chromatin IP Kit (Cell Signaling) according to the manufacturer protocol, followed by 30 seconds of sonication. ChIP was then performed according to the SimpleChIP Enzymatic Chromatin IP Kit (Cell Signaling) with the addition of $5 \mathrm{mM}$ sodium butyrate to preserve histone acetylation. To each $1.25 \mathrm{~mL} \mathrm{IP}$, 1003 the following antibodies were used: 1:125 anti-histone H3.3 (2D7-H1, abnova); 1:50 anti-histone H2A.Z-acetyl (K4/K7) (D3V1I, Cell Signaling); 1:250 anti-histone H2A.Z (ab4174, abcam); 1:50 anti-acetyl-histone H4 (06-866, Millipore); 1:125 anti-histone H4K16ac (Active Motif); 1:50 antiCHD4 (D4B7, Cell Signaling); 1:250 anti-ZMYND8 (A302-089, Bethyl). Crosslinks were 1007 reversed with $0.4 \mathrm{mg} / \mathrm{mL}$ Proteinase $\mathrm{K}$ (ThermoFisher) and $0.2 \mathrm{M} \mathrm{NaCl}$ at $65^{\circ} \mathrm{C}$ for 2 hours. DNA was purified using the ChIP DNA Clean \& Concentrator Kit (Zymo). 
Reske et al.

1013 to IDT for Illumina UDI Adapters (IDT DNA Inc.). Quality and quantity of the finished libraries 1014 were assessed using a combination of Agilent DNA High Sensitivity chip (Agilent Technologies), 1015 QuantiFluor ${ }^{\circledR}$ dsDNA System (Promega) and Kapa Illumina Library Quantification qPCR assays 1016 (Kapa Biosystems). Individually indexed libraries were pooled, and $50 \mathrm{bp}$, paired-end sequencing 1017 (for ZMYND8, H3.3, H2A.Zac, and H4K16ac) or 100 bp, single-end sequencing (for CHD4, 1018 H2A.Z, and pan-H4ac) was performed on an Illumina NovaSeq 6000 sequencer using a 100-cycle 1019 sequencing kit (Illumina). Each library was sequenced to minimum read depth of 80 million reads 1020 per input library and 40 million reads per IP library. Base calling was performed by Illumina NCS 1021 v2.0, and NCS output was demultiplexed and converted to FastQ format with Illumina Bcl2fastq 1022 v1.9.0.

1023 New and re-analyzed (differential) ChIP-seq experiments were analyzed as previously 1024 described (Wilson et al., 2020). Briefly, wild-type CHD4 and differential H2A.Z and pan-H4ac 1025 ChIP-seq experiments were analyzed as single-end libraries, while wild-type ZMYND8 and 1026 differential H3.3, H2A.Zac, and H4K16ac ChIP-seq were analyzed as paired-end libraries. Raw 1027 reads for IPs and inputs were trimmed with cutadapt (Martin, 2011) and Trim Galore! 1028 (http://www.bioinformatics.babraham.ac.uk/projects/trim galore/) followed by quality control 1029 analysis via FastQC (Andrews, 2010) and MultiQC (Ewels et al., 2016). Trimmed reads were 1030 aligned to GRCh38.p12 reference genome (Schneider et al., 2017) via Bowtie2 (Langmead \& 1031 Salzberg, 2012) with flag `--very-sensitive`. Aligned reads were sorted and indexed with samtools 1032 (H. Li et al., 2009). Only properly-paired read fragments were retained for paired-end libraries via 1033 samtools view with flag '-f 3' followed by sorting and indexing. For libraries intended for 1034 differential analyses, molecular complexity was then estimated from duplicate rates by 1035 ATACseqQC (Ou et al., 2018) and preseqR (Daley \& Smith, 2013), and libraries were subsampled 
Reske et al.

1036 to equivalent molecular complexity within an experimental design based on these estimates with 1037 samtools. Picard MarkDuplicates (http://broadinstitute.github.io/picard/) was used to remove 1038 PCR duplicates, followed by sorting and indexing. MACS2 (Y. Zhang et al., 2008) was used to call 1039 peaks on each ChIP replicate against the respective input control. For CHD4 and ZMYND8 IPs, MACS 2 called broadPeaks with FDR $<0.05$ threshold and otherwise default settings. For H2A.Z and H2A.Zac IPs, MACS2 called narrowPeaks with FDR $<0.05$ threshold and flags `--nomodel -extsize $146^{`}$ to bypass model building. For H3.3, pan-H4ac, and H4K16ac IPs, MACS2 called 1043 broadPeaks with FDR $<0.05$ threshold and flags '--nomodel --extsize 146' to bypass model 1044 building. The resulting peaks were repeat-masked by ENCODE blacklist filtering and filtered for non-standard contigs (Amemiya, Kundaje, \& Boyle, 2019). A naive overlapping peak set, as 1046 defined by ENCODE (Landt et al., 2012), was constructed by calling peaks on pooled replicates 1047 followed by bedtools intersect (Quinlan \& Hall, 2010) to select for peaks of at least 50\% overlap 1048 with each biological replicate. 2016). First, a consensus peak set was constructed for each differential experiment from the union 1051 of replicate-intersecting, filtered MACS2 peak regions called in each condition. ChIP reads were 1052 counted in these query regions by csaw, then filtered for low abundance peaks with average $1053 \log _{2} \mathrm{CPM}>-3$. When comparing ChIP libraries, any global differences in IP efficiency observed 1054 between the two conditions were considered a result of technical bias to ensure a highly 1055 conservative analysis. As such, we employed a loess-based local normalization to the peak count 1056 matrix, as is implemented in $\operatorname{csaw}$ (Lun \& Smyth, 2016), to assume a symmetrical MA distribution. 1057 A design matrix was then constructed from one 'condition' variable, without an intercept term. 1058 The count matrix and loess offsets were then supplied to edgeR (M. D. Robinson, McCarthy, \& 
Reske et al.

1059 Smyth, 2010) for estimating dispersions and fitting quasi-likelihood generalized linear models for 1060 differential abundance hypothesis testing. Nearby query regions were then merged up to $500 \mathrm{bp}$ 1061 apart for a maximum merged region width of $5 \mathrm{~kb}$, and the most significant probability was used 1062 to represent the merged region. Finally, FDR $<0.05$ threshold was used to define significant 1063 differentially abundant regions.

\section{Chromatin state modeling and optimization.}

1066 The same genome-wide chromatin 18-state map of $12 \mathrm{Z}$ cells with or without ARID1A depletion, 1067 constructed with ChromHMM (Ernst and Kellis 2012, 2017) using total RNA, ATAC, H3K4me1, 1068 H3K4me3, H3K18ac, H3K27ac, and H3K27me3 data (Wilson et al. 2020), was re-analyzed in 1069 Figure 2 and Figure 3 studies. A refined ChromHMM model was constructed with further addition of H3.3, H2A.Z, H2A.Zac (K4/K7), pan-H4ac (K5/K8/K12/K16), and H4K16ac features with

1071 some procedural modifications. In order to reduce technical confounders in differential chromatin 1072 state analysis between control and ARID1A-depleted cell types, we adopted an equalized 1073 binarization framework described by Fiziev et al. (Fiziev et al. 2017). Briefly, the ChromHMM 1074 chromosomal signal intermediate files during BAM binarization were saved and imported into R. 1075 Feature signal values were then background-subtracted by respective control signals when 1076 available (e.g. input chromatin for ChIP; does not occur for ATAC). For each feature and cell type, 1077 those (background-subtracted) signal values were ranked, and the top $n$ ranked binarization calls 1078 are selected, where $n$ is the lower number of calls among the two cell types for the given feature. 1079 The result is a new equalized binarization, where each feature has the same number of 'present' 1080 region calls in both cell types, per chromosome. As an example, if H3K18ac called 27,000 present 1081 regions on chromosome 1 in control cells and 35,000 present regions in ARID1A-depleted cells, 
Reske et al.

1082 then the top 27,000 regions are retained in both cell types. Chromatin state models from 5 to 40 1083 states were then computed using the "concatenated" approach to unify both cell types for 1084 differential state comparisons. The new chromatin-state model was optimized at 25 states through 1085 a strategy devised by Gorkin et al. (Gorkin et al., 2020), which utilizes the ChromHMM 1086 CompareModels function to compare feature emission parameters from the 40-state (most 1087 complex) model against all other simpler models, as well as a k-means clustering of emission 1088 probabilities from all models together and analyzing the goodness of fit. See Figure 7-figure 1089 supplement 3 for related analyses. Across both strategies, 25 states was observed as a threshold 1090 for $>95 \%$ median maximal state correlation and goodness of fit (between-cluster vs. total sum-of1091 squares) relative to the most complex model.

\section{Bioinformatics and statistics.}

1094 The human endometrioma vs. control endometrium genome-wide expression (Illumina 1095 BeadChips) data set (Hawkins et al., 2011) was retrieved from GEO accession GSE23339 and 1096 analyzed via GEO2R and limma (Barrett et al., 2013; Davis \& Meltzer, 2007; Ritchie et al., 2015). 1097 biomaRt was used for all gene nomenclature and mouse-human ortholog conversions (Smedley et 1098 al., 2009). The cumulative hypergeometric distribution was calculated in R for enrichment tests. 1099 HOMER was used to quantify sequencing reads across sets of genomic regions including heatmaps 1100 (Heinz et al., 2010). GenomicRanges functions were used to intersect and manipulate genomic 1101 coordinates (Lawrence et al., 2013). IGV (J. T. Robinson et al., 2011) was used for visualizing 1102 epigenomic data across hg38 loci as MACS2 enrichment log-likelihood (logLR) for ChIP-seq and 1103 ATAC-seq or FPKM for RNA-seq. Hierarchical clustering by Euclidean distance and heatmaps 1104 were generated by ComplexHeatmap (Gu, Eils, \& Schlesner, 2016). ggplot2 was used for some 
Reske et al.

1105 plots in this study (Wickham, 2016). The statistical language $\mathrm{R}$ was used for various computing 1106 functions throughout this study (R Core Team, 2018).

1108 Funding

1109 RLC was supported by awards from the National Institute of Child Health and Human 1110 Development (R01HD103617 and R21HD099383) from NIH. MRW was supported by a National 1111 Cancer Institute Transition to Independence K99 Award (CA25152) from NIH. JH was supported 1112 by an F32 award (CA260116) from NIH. SBR was supported by an award from the National 1113 Institute of General Medical Sciences (GM124736) from NIH.

\section{Authors' Contributions}

1116 JJR and RLC conceptualized the study. JJR generated, analyzed, and curated all new experimental

1117 genomic data, led integrative computational and statistical analysis, and performed bioinformatic 1118 investigation of public data resources. MRW performed the cell cycle analysis. BA and CP assisted 1119 with experiments. MA led the library construction and sequencing efforts. JH and SBR performed 1120 histone peptide array experiments for antibody characterization. RLC supervised the study and 1121 provided funding. All authors read, critiqued, and approved the final manuscript.

\section{Acknowledgments}

1124 We thank the Van Andel Research Institute Genomics Core for providing sequencing facilities and 1125 services. We thank Dr. Jeremy Prokop for the use of his ÄKTA start FPLC to prepare linear 1126 glycerol gradients. 
Reske et al.

\section{Competing Interests}

1129 The authors declare no competing interests.

\section{References}

1132 Adhikary, S., Sanyal, S., Basu, M., Sengupta, I., Sen, S., Srivastava, D. K., . . Das, C. (2016). Selective Recognition of H3.1K36 Dimethylation/H4K16 Acetylation Facilitates the Regulation of All-trans-retinoic Acid (ATRA)-responsive Genes by Putative Chromatin

Ahmad, K., \& Henikoff, S. (2002). The histone variant H3.3 marks active chromatin by replication-independent nucleosome assembly. Mol Cell, 9(6), 1191-1200. doi:10.1016/s1097-2765(02)00542-7

Alver, B. H., Kim, K. H., Lu, P., Wang, X., Manchester, H. E., Wang, W., . . Roberts, C. W. (2017). The SWI/SNF chromatin remodelling complex is required for maintenance of lineage specific enhancers. Nat. Commun., 8, 14648. doi:10.1038/ncomms14648

Amemiya, H. M., Kundaje, A., \& Boyle, A. P. (2019). The ENCODE Blacklist: Identification of Problematic Regions of the Genome. Sci Rep, 9(1), 9354. doi:10.1038/s41598-019-45839Retrieved from http://www.bioinformatics.babraham.ac.uk/projects/fastqc 
Reske et al.

1151

1152

1153

1154

1155

1156

1157

1158

1159

1160

1161

1162

1163

1164

1165

1166

1167

1168

1169

1170

1171

1172

1173

Barrett, T., Wilhite, S. E., Ledoux, P., Evangelista, C., Kim, I. F., Tomashevsky, M., .. Soboleva, A. (2013). NCBI GEO: archive for functional genomics data sets--update. Nucleic Acids Res, 41(Database issue), D991-995. doi:10.1093/nar/gks1193

Barutcu, A. R., Lajoie, B. R., Fritz, A. J., McCord, R. P., Nickerson, J. A., van Wijnen, A. J., . . Imbalzano, A. N. (2016). SMARCA4 regulates gene expression and higher-order chromatin structure in proliferating mammary epithelial cells. Genome Res, 26(9), 11881201. doi:10.1101/gr.201624.115

Basta, J., \& Rauchman, M. (2015). The nucleosome remodeling and deacetylase complex in development and disease. Transl Res, 165(1), 36-47. doi:10.1016/j.trs1.2014.05.003

Bottardi, S., Mavoungou, L., Pak, H., Daou, S., Bourgoin, V., Lakehal, Y. A., . . Milot, E. (2014). The IKAROS interaction with a complex including chromatin remodeling and transcription elongation activities is required for hematopoiesis. PLoS Genet, 10(12), e1004827. doi:10.1371/journal.pgen.1004827

Bui, C. B., Le, H. K., Vu, D. M., Truong, K. D., Nguyen, N. M., Ho, M. A. N., \& Truong, D. Q. (2019). ARID1A-SIN3A drives retinoic acid-induced neuroblastoma differentiation by transcriptional repression of TERT. Mol Carcinog, 58(11), 1998-2007. doi:10.1002/mc.23091

Cancer Genome Atlas Research, N., Kandoth, C., Schultz, N., Cherniack, A. D., Akbani, R., Liu, Y., . . . Levine, D. A. (2013). Integrated genomic characterization of endometrial carcinoma. Nature, 497(7447), 67-73. doi:10.1038/nature12113

Chandler, R. L., Brennan, J., Schisler, J. C., Serber, D., Patterson, C., \& Magnuson, T. (2013). ARID1a-DNA interactions are required for promoter occupancy by SWI/SNF. Molecular and Cellular Biology, 33(2), 265-280. doi:10.1128/MCB.01008-12 
Reske et al.

1174 Chen, P., Zhao, J., Wang, Y., Wang, M., Long, H., Liang, D., . . . Li, G. (2013). H3.3 actively

1175 marks enhancers and primes gene transcription via opening higher-ordered chromatin. Genes Dev, 27(19), 2109-2124. doi:10.1101/gad.222174.113

1177 Cheneby, J., Menetrier, Z., Mestdagh, M., Rosnet, T., Douida, A., Rhalloussi, W., . . Ballester, B. (2020). ReMap 2020: a database of regulatory regions from an integrative analysis of Human and Arabidopsis DNA-binding sequencing experiments. Nucleic Acids Res,

Clapier, C. R. (2021). Sophisticated Conversations between Chromatin and Chromatin Remodelers, and Dissonances in Cancer. Int J Mol Sci, 22(11). doi:10.3390/ijms22115578

Clapier, C. R., \& Cairns, B. R. (2009). The biology of chromatin remodeling complexes. Annu Rev Biochem, 78, 273-304. doi:10.1146/annurev.biochem.77.062706.153223 Function to H2A.Z Acetylation. iScience, 21, 773-788. doi:10.1016/j.isci.2019.10.053

Cornett, E. M., Dickson, B. M., \& Rothbart, S. B. (2017). Analysis of Histone Antibody Specificity with Peptide Microarrays. J Vis Exp(126). doi:10.3791/55912

Daley, T., \& Smith, A. D. (2013). Predicting the molecular complexity of sequencing libraries. Nat Methods, 10(4), 325-327. doi:10.1038/nmeth.2375 human SWI-SNF complex protein p270 is an ARID family member with non-sequencespecific DNA binding activity. Mol Cell Biol, 20(9), 3137-3146. 
Reske et al.

Davis, S., \& Meltzer, P. S. (2007). GEOquery: a bridge between the Gene Expression Omnibus (GEO) and BioConductor. Bioinformatics, 23(14), 1846-1847. doi:10.1093/bioinformatics/btm254

Deaton, A. M., Gomez-Rodriguez, M., Mieczkowski, J., Tolstorukov, M. Y., Kundu, S., Sadreyev, R. I., . . Kingston, R. E. (2016). Enhancer regions show high histone H3.3 turnover that changes during differentiation. Elife, 5. doi:10.7554/eLife.15316

Dechassa, M. L., Sabri, A., Pondugula, S., Kassabov, S. R., Chatterjee, N., Kladde, M. P., \& Bartholomew, B. (2010). SWI/SNF has intrinsic nucleosome disassembly activity that is dependent on adjacent nucleosomes. Mol Cell, 38(4), 590-602. doi:10.1016/j.molcel.2010.02.040

Dickson, B. M., Cornett, E. M., Ramjan, Z., \& Rothbart, S. B. (2016). ArrayNinja: An Open Source Platform for Unified Planning and Analysis of Microarray Experiments. Methods Enzymol, 574, 53-77. doi:10.1016/bs.mie.2016.02.002

Dobin, A., Davis, C. A., Schlesinger, F., Drenkow, J., Zaleski, C., Jha, S., . . . Gingeras, T. R. (2013). STAR: ultrafast universal RNA-seq aligner. Bioinformatics, $29(1)$, 15-21. doi:10.1093/bioinformatics/bts635

Ernst, J., \& Kellis, M. (2012). ChromHMM: automating chromatin-state discovery and characterization. Nature methods, 9(3), 215-216. doi:10.1038/nmeth.1906

Ewels, P., Magnusson, M., Lundin, S., \& Kaller, M. (2016). MultiQC: summarize analysis results for multiple tools and samples in a single report. Bioinformatics, 32(19), 3047-3048. doi:10.1093/bioinformatics/btw354

Gehre, M., Bunina, D., Sidoli, S., Lubke, M. J., Diaz, N., Trovato, M., . . . Noh, K. M. (2020). Lysine 4 of histone H3.3 is required for embryonic stem cell differentiation, histone 
Reske et al.

enrichment at regulatory regions and transcription accuracy. Nat Genet, 52(3), 273-282. doi:10.1038/s41588-020-0586-5

1221

1222

1223

1224

1225

1226

1227

1228

1229

1230

1231

1232

1233

1234

1235

1236

1237

1238

1239

1240

Ghandi, M., Huang, F. W., Jane-Valbuena, J., Kryukov, G. V., Lo, C. C., McDonald, E. R., 3rd, . . Sellers, W. R. (2019). Next-generation characterization of the Cancer Cell Line Encyclopedia. Nature, 569(7757), 503-508. doi:10.1038/s41586-019-1186-3

Ghosh, K., Tang, M., Kumari, N., Nandy, A., Basu, S., Mall, D. P., . . Biswas, D. (2018). Positive Regulation of Transcription by Human ZMYND8 through Its Association with P-TEFb Complex. Cell Reports, 24(8), 2141-2154 e2146. doi:10.1016/j.celrep.2018.07.064

Gong, F., Chiu, L. Y., Cox, B., Aymard, F., Clouaire, T., Leung, J. W., . . Miller, K. M. (2015). Screen identifies bromodomain protein ZMYND8 in chromatin recognition of transcription-associated DNA damage that promotes homologous recombination. Genes Dev, 29(2), 197-211. doi:10.1101/gad.252189.114

Gong, F., Clouaire, T., Aguirrebengoa, M., Legube, G., \& Miller, K. M. (2017). Histone demethylase KDM5A regulates the ZMYND8-NuRD chromatin remodeler to promote DNA repair. Journal of Cell Biology, 216(7), 1959-1974. doi:10.1083/jcb.201611135

Gorkin, D. U., Barozzi, I., Zhao, Y., Zhang, Y., Huang, H., Lee, A. Y., . . Ren, B. (2020). An atlas of dynamic chromatin landscapes in mouse fetal development. Nature, 583(7818), 744-751. doi:10.1038/s41586-020-2093-3

Grandi, G., Toss, A., Cortesi, L., Botticelli, L., Volpe, A., \& Cagnacci, A. (2015). The Association between Endometriomas and Ovarian Cancer: Preventive Effect of Inhibiting Ovulation and Menstruation during Reproductive Life. Biomed Res Int, 2015, 751571. doi:10.1155/2015/751571 
Reske et al.

1241 Gu, Z., Eils, R., \& Schlesner, M. (2016). Complex heatmaps reveal patterns and correlations in 1242 multidimensional genomic data. Bioinformatics, 32(18), 2847-2849. doi:10.1093/bioinformatics/btw313

1244 Guo, R., Zheng, L., Park, J. W., Lv, R., Chen, H., Jiao, F., . . . Shi, Y. (2014). BS69/ZMYND11 reads and connects histone $\mathrm{H} 3.3$ lysine 36 trimethylation-decorated chromatin to regulated pre-mRNA processing. Molecular Cell, 56(2), 298-310. doi:10.1016/j.molcel.2014.08.022

1247 Ha, M., Kraushaar, D. C., \& Zhao, K. (2014). Genome-wide analysis of H3.3 dissociation reveals high nucleosome turnover at distal regulatory regions of embryonic stem cells. Epigenetics Chromatin, 7(1), 38. doi:10.1186/1756-8935-7-38

Hawkins, S. M., Creighton, C. J., Han, D. Y., Zariff, A., Anderson, M. L., Gunaratne, P. H., \& Matzuk, M. M. (2011). Functional microRNA involved in endometriosis. Mol Endocrinol, 25(5), 821-832. doi:10.1210/me.2010-0371

Hazan, I., Monin, J., Bouwman, B. A. M., Crosetto, N., \& Aqeilan, R. I. (2019). Activation of 1254 Oncogenic Super-Enhancers Is Coupled with DNA Repair by RAD51. Cell Rep, 29(3), 560-572 e564. doi:10.1016/j.celrep.2019.09.001

He, S., Wu, Z., Tian, Y., Yu, Z., Yu, J., Wang, X., . . Xu, Y. (2020). Structure of nucleosomebound human BAF complex. Science, 367(6480), 875-881. doi:10.1126/science.aaz9761

1258 Heinz, S., Benner, C., Spann, N., Bertolino, E., Lin, Y. C., Laslo, P., . . . Glass, C. K. (2010). elements required for macrophage and B cell identities. Mol Cell, 38(4), 576-589. doi:10.1016/j.molcel.2010.05.004 
Reske et al.

1262 Ignatiadis, N., Klaus, B., Zaugg, J. B., \& Huber, W. (2016). Data-driven hypothesis weighting 1263 increases detection power in genome-scale multiple testing. Nat. Methods, 13(7), 577-580.

Jiao, F., Li, Z., He, C., Xu, W., Yang, G., Liu, T., . . . Guo, R. (2020). RACK7 recognizes H3.3G34R mutation to suppress expression of MHC class II complex components and their delivery pathway in pediatric glioblastoma. Sci Adv, 6(29), eaba2113.

Jones, S., Wang, T. L., Shih Ie, M., Mao, T. L., Nakayama, K., Roden, R., . . Papadopoulos, N. (2010). Frequent mutations of chromatin remodeling gene ARID1A in ovarian clear cell carcinoma. Science, 330(6001), 228-231. doi:10.1126/science.1196333

1272 Kadoch, C., \& Crabtree, G. R. (2015). Mammalian SWI/SNF chromatin remodeling complexes and cancer: Mechanistic insights gained from human genomics. Sci Adv, 1(5), e1500447. doi:10.1126/sciadv.1500447

1275 Kadoch, C., Hargreaves, D. C., Hodges, C., Elias, L., Ho, L., Ranish, J., \& Crabtree, G. R. (2013). Proteomic and bioinformatic analysis of mammalian SWI/SNF complexes identifies extensive roles in human malignancy. Nature Genetics, 45(6), 592-601. doi:10.1038/ng.2628

1279 Kassabov, S. R., Zhang, B., Persinger, J., \& Bartholomew, B. (2003). SWI/SNF unwraps, slides, and rewraps the nucleosome. Mol Cell, 11(2), 391-403. doi:10.1016/s1097-

1282 Kelso, T. W. R., Porter, D. K., Amaral, M. L., Shokhirev, M. N., Benner, C., \& Hargreaves, D. C. (2017). Chromatin accessibility underlies synthetic lethality of SWI/SNF subunits in ARID1A-mutant cancers. Elife, 6. doi:10.7554/eLife.30506 
Reske et al.

1285

1286

1287

1288

1289

1290

1291

1292

1293

1294

1295

1296

1297

1298

1299

1300

1301

1302

1303

1304

1305

1306

1307

Konev, A. Y., Tribus, M., Park, S. Y., Podhraski, V., Lim, C. Y., Emelyanov, A. V., .. . Fyodorov, D. V. (2007). CHD1 motor protein is required for deposition of histone variant H3.3 into chromatin in vivo. Science, 317(5841), 1087-1090. doi:10.1126/science.1145339

Kraushaar, D. C., Chen, Z., Tang, Q., Cui, K., Zhang, J., \& Zhao, K. (2018). The gene repressor complex NuRD interacts with the histone variant $\mathrm{H} 3.3$ at promoters of active genes. Genome Res, 28(11), 1646-1655. doi:10.1101/gr.236224.118

Landt, S. G., Marinov, G. K., Kundaje, A., Kheradpour, P., Pauli, F., Batzoglou, S., .. . Snyder, M. (2012). ChIP-seq guidelines and practices of the ENCODE and modENCODE consortia. Genome Res, 22(9), 1813-1831. doi:10.1101/gr.136184.111

Langmead, B., \& Salzberg, S. L. (2012). Fast gapped-read alignment with Bowtie 2. Nat Methods, 9(4), 357-359. doi:10.1038/nmeth. 1923

Lawrence, M., Huber, W., Pages, H., Aboyoun, P., Carlson, M., Gentleman, R., . . Carey, V. J. (2013). Software for computing and annotating genomic ranges. PLoS Comput Biol, 9(8), e1003118. doi:10.1371/journal.pcbi.1003118

Li, H., Handsaker, B., Wysoker, A., Fennell, T., Ruan, J., Homer, N., . . Genome Project Data Processing, S. (2009). The Sequence Alignment/Map format and SAMtools. Bioinformatics, 25(16), 2078-2079. doi:10.1093/bioinformatics/btp352

Li, N., Li, Y., Lv, J., Zheng, X., Wen, H., Shen, H., . . Lee, M. G. (2016). ZMYND8 Reads the Dual Histone Mark H3K4me1-H3K14ac to Antagonize the Expression of MetastasisLinked Genes. Molecular Cell, 63(3), 470-484. doi:10.1016/j.molcel.2016.06.035

Local, A., Huang, H., Albuquerque, C. P., Singh, N., Lee, A. Y., Wang, W., . . Ren, B. (2018). Identification of $\mathrm{H} 3 \mathrm{~K} 4 \mathrm{me} 1$-associated proteins at mammalian enhancers. Nat Genet, 50(1), 73-82. doi:10.1038/s41588-017-0015-6 
Reske et al.

1308

1309

1310

1311

1312

1313

1314

1315

1316

1317

1318

1319

1320

1321

1322

1323

1324

1325

1326

1327

1328

Love, M. I., Anders, S., Kim, V., \& Huber, W. (2015). RNA-Seq workflow: gene-level exploratory analysis and differential expression. F1000Res, 4, 1070. doi:10.12688/f1000research.7035.1

Love, M. I., Huber, W., \& Anders, S. (2014). Moderated estimation of fold change and dispersion for RNA-seq data with DESeq2. Genome Biology, 15(12), 550. doi:10.1186/s13059-014$0550-8$

Lun, A. T., \& Smyth, G. K. (2016). csaw: a Bioconductor package for differential binding analysis of ChIP-seq data using sliding windows. Nucleic Acids Res, 44(5), e45. doi:10.1093/nar/gkv1191

Marques, J. G., Gryder, B. E., Pavlovic, B., Chung, Y., Ngo, Q. A., Frommelt, F., . . Schafer, B. W. (2020). NuRD subunit CHD4 regulates super-enhancer accessibility in rhabdomyosarcoma and represents a general tumor dependency. Elife, 9. doi:10.7554/eLife.54993

Martin, M. (2011). Cutadapt removes adapter sequences from high-throughput sequencing reads. EMBnet. J., 17, 10-12.

Martire, S., Gogate, A. A., Whitmill, A., Tafessu, A., Nguyen, J., Teng, Y. C., . . Banaszynski, L. A. (2019). Phosphorylation of histone H3.3 at serine 31 promotes p300 activity and enhancer acetylation. Nat Genet, 51(6), 941-946. doi:10.1038/s41588-019-0428-5

Mashtalir, N., D'Avino, A. R., Michel, B. C., Luo, J., Pan, J., Otto, J. E., . . Kadoch, C. (2018). Modular Organization and Assembly of SWI/SNF Family Chromatin Remodeling Complexes. Cell, 175(5), 1272-1288 e1220. doi:10.1016/j.cell.2018.09.032 
Reske et al.

1329

1330

1331

1332

1333

1334

1335

1336

1337

1338

1339

1340

1341

1342

1343

1344

1345

1346

1347

1348

1349

1350

Mittal, P., \& Roberts, C. W. M. (2020). The SWI/SNF complex in cancer - biology, biomarkers and therapy. Nature Reviews: Clinical Oncology, 17(7), 435-448. doi:10.1038/s41571020-0357-3

Morris, S. A., Baek, S., Sung, M. H., John, S., Wiench, M., Johnson, T. A., . . Hager, G. L. (2014). Overlapping chromatin-remodeling systems collaborate genome wide at dynamic chromatin transitions. Nat Struct Mol Biol, 21(1), 73-81. doi:10.1038/nsmb.2718

Nacev, B. A., Feng, L., Bagert, J. D., Lemiesz, A. E., Gao, J., Soshnev, A. A., . . Allis, C. D. (2019). The expanding landscape of 'oncohistone' mutations in human cancers. Nature, 567(7749), 473-478. doi:10.1038/s41586-019-1038-1

Ou, J., Liu, H., Yu, J., Kelliher, M. A., Castilla, L. H., Lawson, N. D., \& Zhu, L. J. (2018). ATACseqQC: a Bioconductor package for post-alignment quality assessment of ATACseq data. BMC Genomics, 19(1), 169. doi:10.1186/s12864-018-4559-3

Park, J. H., Park, E. J., Lee, H. S., Kim, S. J., Hur, S. K., Imbalzano, A. N., \& Kwon, J. (2006). Mammalian SWI/SNF complexes facilitate DNA double-strand break repair by promoting gamma-H2AX induction. Embo J, 25(17), 3986-3997. doi:10.1038/sj.emboj.7601291

Pillidge, Z., \& Bray, S. J. (2019). SWI/SNF chromatin remodeling controls Notch-responsive enhancer accessibility. EMBO Rep, 20(5). doi:10.15252/embr.201846944

Pradhan, S. K., Su, T., Yen, L., Jacquet, K., Huang, C., Cote, J., . . Carey, M. F. (2016). EP400 Deposits H3.3 into Promoters and Enhancers during Gene Activation. Mol Cell, 61(1), 2738. doi:10.1016/j.molcel.2015.10.039

Quinlan, A. R., \& Hall, I. M. (2010). BEDTools: a flexible suite of utilities for comparing genomic features. Bioinformatics, 26(6), 841-842. doi:10.1093/bioinformatics/btq033 
Reske et al.

1351 R Core Team. (2018). R: A language and environment for statistical computing. Retrieved from https://www.R-project.org/

1353

1354

1355

1356

1357

1358

1359

1360

1361

1362

1363

1364

1365

1366

1367

1368

1369

1370

1371

1372

Rafati, H., Parra, M., Hakre, S., Moshkin, Y., Verdin, E., \& Mahmoudi, T. (2011). Repressive LTR nucleosome positioning by the BAF complex is required for HIV latency. PLoS Biol, 9(11), e1001206. doi:10.1371/journal.pbio.1001206

Reske, J. J., Wilson, M. R., Holladay, J., Wegener, M., Adams, M., \& Chandler, R. L. (2020). SWI/SNF inactivation in the endometrial epithelium leads to loss of epithelial integrity. Human Molecular Genetics, 29(20), 3412-3430. doi:10.1093/hmg/ddaa227

Ritchie, M. E., Phipson, B., Wu, D., Hu, Y., Law, C. W., Shi, W., \& Smyth, G. K. (2015). limma powers differential expression analyses for RNA-sequencing and microarray studies. Nucleic Acids Res, 43(7), e47. doi:10.1093/nar/gkv007

Robinson, J. T., Thorvaldsdottir, H., Winckler, W., Guttman, M., Lander, E. S., Getz, G., \& Mesirov, J. P. (2011). Integrative genomics viewer. Nat Biotechnol, 29(1), 24-26. doi:10.1038/nbt. 1754

Robinson, M. D., McCarthy, D. J., \& Smyth, G. K. (2010). edgeR: a Bioconductor package for differential expression analysis of digital gene expression data. Bioinformatics, 26(1), 139140. doi:10.1093/bioinformatics/btp616

Rothbart, S. B., Krajewski, K., Strahl, B. D., \& Fuchs, S. M. (2012). Peptide microarrays to interrogate the "histone code". Methods Enzymol, 512, 107-135. doi:10.1016/B978-0-12391940-3.00006-8

Samartzis, E. P., Samartzis, N., Noske, A., Fedier, A., Caduff, R., Dedes, K. J., . . Imesch, P. (2012). Loss of ARID1A/BAF250a-expression in endometriosis: a biomarker for risk of 
Reske et al.

carcinogenic transformation? Modern Pathology, 25(6), 885-892. doi:10.1038/modpathol.2011.217

1375

1376

1377

1378

1379

1380

1381

1382

1383

1384

1385

1386

1387

1388

1389

1390

1391

1392

1393

1394

1395

Savitsky, P., Krojer, T., Fujisawa, T., Lambert, J. P., Picaud, S., Wang, C. Y., . . Filippakopoulos, P. (2016). Multivalent Histone and DNA Engagement by a PHD/BRD/PWWP Triple Reader Cassette Recruits ZMYND8 to K14ac-Rich Chromatin. Cell Rep, 17(10), 27242737. doi:10.1016/j.celrep.2016.11.014

Schneider, V. A., Graves-Lindsay, T., Howe, K., Bouk, N., Chen, H. C., Kitts, P. A., ... Church, D. M. (2017). Evaluation of GRCh38 and de novo haploid genome assemblies demonstrates the enduring quality of the reference assembly. Genome Res, 27(5), 849-864. doi:10.1101/gr.213611.116

Schwabish, M. A., \& Struhl, K. (2007). The Swi/Snf complex is important for histone eviction during transcriptional activation and RNA polymerase II elongation in vivo. Mol Cell Biol, 27(20), 6987-6995. doi:10.1128/MCB.00717-07

Serresi, M., Kertalli, S., Li, L., Schmitt, M. J., Dramaretska, Y., Wierikx, J., . . Gargiulo, G. (2021). Functional antagonism of chromatin modulators regulates epithelial-mesenchymal transition. Sci Adv, 7(9). doi:10.1126/sciadv.abd7974

Shen, H., Xu, W., Guo, R., Rong, B., Gu, L., Wang, Z., .. . Lan, F. (2016). Suppression of Enhancer Overactivation by a RACK7-Histone Demethylase Complex. Cell, 165(2), 331-342. doi:10.1016/j.cell.2016.02.064

Shi, L., Wen, H., \& Shi, X. (2017). The Histone Variant H3.3 in Transcriptional Regulation and Human Disease. J Mol Biol, 429(13), 1934-1945. doi:10.1016/j.jmb.2016.11.019

Sikora, J., Wroblewska-Czech, A., Smycz-Kubanska, M., Mielczarek-Palacz, A., Cygal, A., Witek, A., \& Kondera-Anasz, Z. (2018). The role of complement components C1q, MBL 
Reske et al.

and C1 inhibitor in pathogenesis of endometriosis. Arch Gynecol Obstet, 297(6), 14951501. doi:10.1007/s00404-018-4754-0

Smedley, D., Haider, S., Ballester, B., Holland, R., London, D., Thorisson, G., \& Kasprzyk, A. (2009). BioMart--biological queries made easy. BMC Genomics, 10, 22. doi:10.1186/1471-2164-10-22

1401

1402

1403

1404

1405

1406

1407

1408

1409

1410

1411

1412

1413

1414

1415

1416

1417

1418

Smeenk, G., Wiegant, W. W., Vrolijk, H., Solari, A. P., Pastink, A., \& van Attikum, H. (2010). The NuRD chromatin-remodeling complex regulates signaling and repair of DNA damage. J Cell Biol, 190(5), 741-749. doi:10.1083/jcb.201001048

Spruijt, C. G., Luijsterburg, M. S., Menafra, R., Lindeboom, R. G., Jansen, P. W., Edupuganti, R. R., . . Vermeulen, M. (2016). ZMYND8 Co-localizes with NuRD on Target Genes and Regulates Poly(ADP-Ribose)-Dependent Recruitment of GATAD2A/NuRD to Sites of DNA Damage. Cell Rep, 17(3), 783-798. doi:10.1016/j.celrep.2016.09.037

Szenker, E., Ray-Gallet, D., \& Almouzni, G. (2011). The double face of the histone variant H3.3. Cell Res, 21(3), 421-434. doi:10.1038/cr.2011.14

Tong, J. K., Hassig, C. A., Schnitzler, G. R., Kingston, R. E., \& Schreiber, S. L. (1998). Chromatin deacetylation by an ATP-dependent nucleosome remodelling complex. Nature, 395(6705), 917-921. doi:10.1038/27699

Trizzino, M., Barbieri, E., Petracovici, A., Wu, S., Welsh, S. A., Owens, T. A., . . Gardini, A. (2018). The Tumor Suppressor ARID1A Controls Global Transcription via Pausing of RNA Polymerase II. Cell Reports, 23(13), 3933-3945. doi:10.1016/j.celrep.2018.05.097

Van Rechem, C., Boulay, G., \& Leprince, D. (2009). HIC1 interacts with a specific subunit of SWI/SNF complexes, ARID1A/BAF250A. Biochem Biophys Res Commun, 385(4), 586590. doi:10.1016/j.bbrc.2009.05.115 
Reske et al.

1419 Wang, W., Cote, J., Xue, Y., Zhou, S., Khavari, P. A., Biggar, S. R., . . Crabtree, G. R. (1996).

1423 Wang, W., Vilella, F., Alama, P., Moreno, I., Mignardi, M., Isakova, A., . . Quake, S. R. (2020).

Wen, H., Li, Y., Xi, Y., Jiang, S., Stratton, S., Peng, D., . . Shi, X. (2014). ZMYND11 links histone $\mathrm{H} 3.3 \mathrm{~K} 36 \mathrm{me} 3$ to transcription elongation and tumour suppression. Nature, 508(7495), 263-268. doi:10.1038/nature13045

1429

1430 Med, 26(10), 1644-1653. doi:10.1038/s41591-020-1040-Z

Whitehouse, I., Flaus, A., Cairns, B. R., White, M. F., Workman, J. L., \& Owen-Hughes, T. (1999). Nucleosome mobilization catalysed by the yeast SWI/SNF complex. Nature, 400(6746), 784-787. doi:10.1038/23506

Whyte, W. A., Orlando, D. A., Hnisz, D., Abraham, B. J., Lin, C. Y., Kagey, M. H., . . Young, R. A. (2013). Master transcription factors and mediator establish super-enhancers at key cell identity genes. Cell, 153(2), 307-319. doi:10.1016/j.cell.2013.03.035

Wickham, H. (2016). ggplot2: Elegant Graphics for Data Analysis: Springer-Verlag New York.

Wiegand, K. C., Shah, S. P., Al-Agha, O. M., Zhao, Y., Tse, K., Zeng, T., . . Huntsman, D. G. (2010). ARID1A mutations in endometriosis-associated ovarian carcinomas. $N$ Engl $J$ Med, 363(16), 1532-1543. doi:10.1056/NEJMoa1008433

Wilson, M. R., Reske, J. J., Holladay, J., Neupane, S., Ngo, J., Cuthrell, N., . . Chandler, R. L. (2020). ARID1A Mutations Promote P300-Dependent Endometrial Invasion through 
Reske et al.

Super-Enhancer Hyperacetylation. Cell Reports, 33(6), 108366. doi:10.1016/j.celrep.2020.108366

1443 Wilson, M. R., Reske, J. J., Holladay, J., Wilber, G. E., Rhodes, M., Koeman, J., . . Chandler, R. L. (2019). ARID1A and PI3-kinase pathway mutations in the endometrium drive epithelial transdifferentiation and collective invasion. Nat Commun, 10(1), 3554. doi:10.1038/s41467-019-11403-6

1447 Wu, R. C., Wang, T. L., \& Shih Ie, M. (2014). The emerging roles of ARID1A in tumor suppression. Cancer Biology \& Therapy, 15(6), 655-664. doi:10.4161/cbt.28411

1449 Xue, Y., Wong, J., Moreno, G. T., Young, M. K., Cote, J., \& Wang, W. (1998). NURD, a novel complex with both ATP-dependent chromatin-remodeling and histone deacetylase activities. Mol Cell, 2(6), 851-861. doi:10.1016/s1097-2765(00)80299-3

1452 Zhang, C., Wang, X., Anaya, Y., Parodi, L., Cheng, L., Anderson, M. L., \& Hawkins, S. M. (2018). Distinct molecular pathways in ovarian endometrioid adenocarcinoma with concurrent endometriosis. Int J Cancer, 143(10), 2505-2515. doi:10.1002/ijc.31768

1455 Zhang, H., Gan, H., Wang, Z., Lee, J. H., Zhou, H., Ordog, T., . . . Zhang, Z. (2017). RPA Interacts 1456 with HIRA and Regulates H3.3 Deposition at Gene Regulatory Elements in Mammalian Cells. Mol Cell, 65(2), 272-284. doi:10.1016/j.molcel.2016.11.030

1458 Zhang, Y., Liu, T., Meyer, C. A., Eeckhoute, J., Johnson, D. S., Bernstein, B. E., . . Liu, X. S. (2008). Model-based analysis of ChIP-Seq (MACS). Genome Biol, 9(9), R137. doi:10.1186/gb-2008-9-9-r137

1461 Zhou, Y., \& Grummt, I. (2005). The PHD finger/bromodomain of NoRC interacts with acetylated histone H4K16 and is sufficient for rDNA silencing. Curr Biol, 15(15), 1434-1438. doi:10.1016/j.cub.2005.06.057 
Reske et al.

Zhu, A., Ibrahim, J. G., \& Love, M. I. (2019). Heavy-tailed prior distributions for sequence count 2092. doi:10.1093/bioinformatics/bty895

Zondervan, K. T., Becker, C. M., \& Missmer, S. A. (2020). Endometriosis. NEngl J Med, 382(13), 1244-1256. doi:10.1056/NEJMra1810764

\section{Source Data Files}

1471 Figure 3-figure supplement 1-source data 1.tif. ARID1A Western Blot raw image as in Figure

14723 -figure supplement 1A and Figure 3-figure supplement 2.

1473 Figure 3-figure supplement 1-source data 2.tif. $\beta$-actin Western Blot raw image as in Figure

14743 -figure supplement 1A and Figure 3-figure supplement 2.

1475 Figure 3-figure supplement 1-source data 3.tif. ARID1A Western Blot raw image as in Figure

14763 -figure supplement 1B and Figure 3-figure supplement 2.

1477 Figure 3-figure supplement 1-source data 4.tif. $\beta$-actin Western Blot raw image as in Figure

$1478 \quad 3$-figure supplement 1B and Figure 3-figure supplement 2.

1479 Figure 3-figure supplement 1-source data 5.tif. Histone H3.3 Western Blot raw image as in

1480 Figure 3-figure supplement 1B and Figure 3-figure supplement 2.

1481 Figure 3-figure supplement 1-source data 6.tif. Total histone H3 Western Blot raw image

1482 (right blot) as in Figure 3-figure supplement 1B and Figure 3-figure supplement 2.

1483 Figure 4-source data 1.tif. Grayscale, high contrast histone H3.3 (left) and total histone H3

1484 (right) Western Blot raw image as in Figure 4B and Figure 4-figure supplement 2. Presented 1485 image is flipped vertically. 
Reske et al.

1486 Figure 4-source data 2.tif. Grayscale, low contrast histone H3.3 (left) and total histone H3

1487 (right) Western Blot raw image as in Figure 4B and Figure 4-figure supplement 2. Presented

1488 image is flipped vertically.

1489 Figure 4-source data 3.tif. Dual-color fluorescent histone H3.3 (left) and total histone H3

1490 (right) Western Blot raw image as in Figure 4-figure supplement 2. Presented image is flipped

1491 vertically.

1492 Figure 5-source data 1.tif. ARID1A Western Blot raw image as in Figure 5C and Figure 5-

1493 figure supplement 1.

1494 Figure 5-source data 2.tif. CHD4 Western Blot raw image as in Figure 5C and Figure 5-figure

1495 supplement 1.

1496 Figure 5-source data 3.tif. ZMYND8 Western Blot raw image as in Figure 5C and Figure 5-

1497 figure supplement 1.

1498 Figure 5-source data 4.tif. CHD4 Western Blot raw image as in Figure 5D and Figure 5-

1499 figure supplement 1.

1500 Figure 5-source data 5.tif. ARID1A Western Blot raw image as in Figure 5D and Figure 5-

1501 figure supplement 1.

1502 Figure 5-source data 6.tif. ZMYND8 Western Blot raw image as in Figure 5D and Figure 5-

1503 figure supplement 1.

1504 Figure 5-source data 7.tif. ARID1A Western Blot raw image as in Figure 5E and Figure 5-

1505 figure supplement 1.

1506 Figure 5-source data 8.tif. BRG1 Western Blot raw image as in Figure 5E and Figure 5-figure

1507 supplement 1. 
Reske et al.

1508 Figure 5-source data 9.tif. BAF155 Western Blot raw image as in Figure 5E and Figure 5-

1509 figure supplement 1.

1510 Figure 5-source data 10.tif. ZMYND8 Western Blot raw image as in Figure 5E and Figure 5-

1511 figure supplement 1.

1512 Figure 5-source data 11.tif. CHD4 Western Blot raw image as in Figure 5E and Figure 5-

1513 figure supplement 1.

1514 Figure 5-source data 12.tif. HDAC1 Western Blot raw image as in Figure 5E and Figure 5-

1515 figure supplement 1.

1516 Figure 7-figure supplement 6-source data 1.tif. ZMYND8 Western Blot raw image as in

1517 Figure 7-figure supplement 6A and Figure 7-figure supplement 9.

1518 Figure 7-figure supplement 6-source data 2.tif. $\beta$-actin Western Blot raw image as in Figure

1519 7-figure supplement 6A and Figure 7-figure supplement 9.

1520 Figure 7-figure supplement 6-source data 3.tif. CHD4 Western Blot raw image as in Figure

15217 -figure supplement 6D and Figure 7-figure supplement 9.

1522 Figure 7-figure supplement 6-source data 4.tif. $\beta$-actin Western Blot raw image as in Figure

15237 -figure supplement 6D and Figure 7-figure supplement 9. 